\title{
A review of the matrix-exponential formalism in radiative transfer
}

\author{
Dmitry S. Efremenko*, Víctor Molina García, Sebastián Gimeno García ${ }^{1}$, Adrian Doicu \\ Deutsches Zentrum für Luft- und Raumfahrt (DLR), Institut für Methodik der Fernerkundung (IMF), 82234 Oberpfaffenhofen, Germany
}

\begin{abstract}
This paper outlines the matrix exponential description of radiative transfer. The eigendecomposition method which serves as a basis for computing the matrix exponential and for representing the solution in a discrete ordinate setting is considered. The mathematical equivalence of the discrete ordinate method, the matrix operator method, and the matrix Riccati equations method is proved rigorously by means of the matrix exponential formalism. For optically thin layers approximate solution methods relying on the Padé and Taylor series approximations to the matrix exponential, as well as on the matrix Riccati equations are presented. For optically thick layers, the asymptotic theory with higher-order corrections is derived, and parametrizations of the asymptotic functions and constants for a water-cloud model with a Gamma size distribution are obtained.
\end{abstract}

Keywords: matrix-exponential, discrete ordinate method, matrix operator method, matrix Riccati equations

\section{Contents}

1 Introduction

2 Matrix formulation of the radiative transfer equation

3 Eigendecomposition method for computing the matrix exponential

4 Discrete ordinate method with matrix exponential

5 Matrix operator method with matrix exponential

5.1 Reflection and transmission matrices of a homogeneous layer . . . . . . . . . . . .

5.2 Discrete approximations of the reflection and transmission functions of a homogeneous layer

5.3 Reflection and transmission matrices of a homogeneous thin layer . . . . . . . . . . . . . . 12

5.3 .1 Padé approximation . . . . . . . . . 12

5.3.2 Taylor series approximation . . . . . . . . 13

5.4 Reflection and transmission matrices of a homogeneous thick layer . . . . . . . . . . . . . 14

5.4.1 Asymptotic theory . . . . . . . . . . . 14

5.4 .2 Higher-order corrections . . . . . . . . 17

5.4.3 Waterman's approximation ...... 18

6 Matrix Riccati equations

6.1 Reflection and transmission matrices of a homogeneous layer . . . . . . . . . . . .

6.2 Approximations based on matrix Riccati equations ....................... 21

6.2.1 Successive orders of scattering . . . . . . 21

6.2.2 Iterative approximation $\ldots \ldots \ldots 22$

\footnotetext{
* Corresponding author

Email address: dmitry . efremenko@dlr.de (Dmitry S. Efremenko)

${ }^{1}$ Now at EUMETSAT, Eumetsat Allee 1, D-64295 Darmstadt, Germany.
}

\section{Conclusions}

Appendix 2. Spectral decomposition of the layer matrix $\mathbf{2 4}$ Direct decomposition method . . . . . . . . . . . 24 Square-root method ..................... 25 Cholesky method . . . . . . . . . . . . 25

Appendix 3. Conservative scattering 25 Basic results . . . . . . . . . . . . 26 Computation of the vectors $\mathbf{w}_{0}$ and $\mathbf{w}_{1} \ldots \ldots$. . . 27 Analytical formulas for conservative scattering . . . 27 A special system of characteristic solutions . . . . . 28

Appendix 4. Asymptotic functions and constants $\quad 29$

References

\section{Introduction}

The radiative transfer is an important issue for astrophysics, atmospheric physics, meteorology and engineering sciences. A wide range of solution methods of the radiative transfer equation (RTE) have been proposed (see, e.g., [111] and references therein for a general review). The discrete ordinate method [6, 12-14] and the matrix operator method [15-18] involve replacing the continuous dependence of the radiance on direction by a dependence on a discrete set of directions. For a homogeneous layer, the discretized radiative transfer equation then takes the form of a system of linear first-order differential equations. In the classical discrete ordinate method of Chandrasekhar, the solution of the system of equations is expressed as a linear combination of characteristic solutions of the discretized problem, while the matrix operator method is primarily oriented toward numeri- 
cal computations of the reflection and transmission matrices. Another group of methods are based on the concept of invariant imbedding, which is due to Ambarzumian [19]. Ambarzumian derived an equation for the reflection function of a semi-infinite atmosphere by noting that the reflection function remains unchanged upon addition of a new layer. This technique was further generalized by Chandrasekhar [13] to a finite layer, while Bellman et al. [20] showed that the reflection function derived by using the invariant imbedding satisfies the Riccati equation.

The system of differential equations of the discretized radiative transfer equation can be solved by using a classical mathematical procedure involving the matrix exponential operator, in which the system matrix appears in the exponent. Waterman [21] was the first who provided a matrix exponential description of radiative transfer. Mathematical elegance aside, he showed its practical value in radiative transfer computations from both the analytical and purely numerical point of view. In particular, Waterman related the matrix exponential to the extinction matrix incorporating the reflection and transmission matrices of a homogeneous layer, provided an eigenvector representation of the matrix exponential, derived analytical expressions for the reflection and transmission matrices in the limit of small and large optical thicknesses, showed that the matrix exponential can be used to generate starting values for the doubling method, and applied the matrix exponential formalism to conservative scattering. Flatau and Stephens [22] extended the concept of matrix exponential of a homogeneous layer to an inhomogeneous atmosphere by introducing the so-called propagator (matrix) operator. As Waterman, Flatau and Stephens related the propagator to the extinction matrix of a homogeneous layer, notified the similarity between the matrix exponential solution and Chandrasekhar's discrete ordinate solution, established various properties of the propagator and used them to derive the Riccati matrix equations for an inhomogenous atmosphere, as well as the adding and doubling formulas. Although in both papers [21, 22] an eigendecomposition method for computing the matrix exponential is considered, explicit and stable representations of the reflection and transmission matrices are not given. This problem has been solved by Nakajima and Tanaka [18] by using a system of characteristic solutions of the discretized problem, and by Budak et al. [23, 24] by using the matrix exponential formalism. It should be also mentioned that Doicu and Trautmann $[25,26]$ designed the so-called discrete ordinate method with matrix exponential to compute the radiance field in a multilayered atmosphere.

The purpose of this paper is to provide a consistent overview of the matrix exponential description of radiative transfer. We mainly focus on a mathematical rigorous and self-contained analysis based on the results given in [21, 22, 27] and our own results [25, 26, 28, 29]. The final goals are to prove the mathematical equivalence of the discrete ordinate method, matrix operator method, and the matrix Riccati equations method, on the one hand, and to derive efficient computations formulas for the reflection and transmission matrices in the limit of small and large optical thicknesses, on the other hand.

The rest of the paper is organized as follows. In Section 2, we present the discrete ordinate setting in which the matrix exponential method is applied, while in Section 3, we discuss the eigendecomposition method for computing the matrix exponential. Section 4 is devoted the discrete ordinate method with matrix exponential. In Section 5, dealing with the matrix operator method with matrix exponential, we derive several representations of the reflection and transmission matrices for arbitrary optical thickness, as well as, for small and large optical thicknesses. In Section 6 we establish the matrix Riccati equations, prove the mathematical equivalence between the matrix Riccati equations method and the matrix exponential method in computing the reflection and transmission matrices of a homogeneous layer, and discuss some approximation solution methods for small values of the optical thickness and/or single scattering albedo. Finally, Section 7 contains some concluding remarks. Additional results dealing with a justification of the Gaussian quadrature in the discrete ordinate method, a review of eigendecomposition methods for computing the matrix exponential, and an extension of the analytical results to conservative scattering are presented in appendices.

\section{Matrix formulation of the radiative transfer equation}

For a given solar direction $\boldsymbol{\Omega}_{0}=\left(-\mu_{0}, \varphi_{0}\right)$, with $\mu_{0}>0$ being the cosine of the solar zenith angle and $\varphi_{0}$ the solar azimuthal angle, the equation describing the radiative transfer in a plane-parallel homogeneous layer of optical thickness $\bar{\tau}$ is

$$
\begin{aligned}
& \mu \frac{\mathrm{d} I_{\mathrm{d}}\left(\tau, \mu,-\mu_{0}, \varphi-\varphi_{0}\right)}{\mathrm{d} \tau} \\
& =I_{\mathrm{d}}\left(\tau, \mu,-\mu_{0}, \varphi-\varphi_{0}\right)-\frac{\omega}{4 \pi} F_{0} p\left(\mu,-\mu_{0}, \varphi-\varphi_{0}\right) \mathrm{e}^{-\tau / \mu_{0}} \\
& -\frac{\omega}{4 \pi} \int_{0}^{2 \pi} \int_{-1}^{1} p\left(\mu, \mu^{\prime}, \varphi-\varphi^{\prime}\right) I_{\mathrm{d}}\left(\tau, \mu^{\prime},-\mu_{0} \varphi^{\prime}-\varphi_{0}\right) \mathrm{d} \mu^{\prime} \mathrm{d} \varphi^{\prime},
\end{aligned}
$$

where $I_{\mathrm{d}}\left(\tau, \mu,-\mu_{0}, \varphi-\varphi_{0}\right)$ is the diffuse radiance at optical depth $\tau$ along the direction specified by the cosine of the zenith angle $\mu$ and the azimuthal angle $\varphi, p\left(\mu, \mu^{\prime}, \varphi-\varphi^{\prime}\right)$ is the scattering phase function for the radiation scattered from the direction $\boldsymbol{\Omega}^{\prime}=\left(\mu^{\prime}, \varphi^{\prime}\right)$ into the direction $\boldsymbol{\Omega}=(\mu, \varphi), \omega$ is the single scattering albedo, and $F_{0}$ is the solar flux. For simplicity, the thermal emission term is neglected in equation (1). If the homogeneous layer is placed in a multi-layered atmosphere at optical depth $\tau_{0}$, the radiative transfer equation for the diffuse radiance e $I_{\mathrm{d}}\left(\tau_{0}+\tau, \mu,-\mu_{0}, \varphi-\varphi_{0}\right)$ contains the direct transmission term $\exp \left[-\left(\tau_{0}+\tau\right) / \mu_{0}\right]$ instead of $\exp \left(-\tau / \mu_{0}\right)$. The total radiance, defined in terms of the diffuse and direct radiances by

$I\left(\tau, \mu,-\mu_{0}, \varphi-\varphi_{0}\right)=I_{\mathrm{d}}\left(\tau, \mu,-\mu_{0}, \varphi-\varphi_{0}\right)+I_{\odot}\left(\tau, \mu,-\mu_{0}, \varphi-\varphi_{0}\right)$

solves the radiative transfer equation (1) without the single scattering source term. Note that for the direct radiance $I_{\odot}$, 
we have

$I_{\odot}\left(\tau, \mu,-\mu_{0}, \varphi-\varphi_{0}\right)=F_{0} \delta\left(\mu+\mu_{0}\right) \delta\left(\varphi-\varphi_{0}\right) \mathrm{e}^{-\tau / \mu_{0}}$,

and

$\mu \frac{\mathrm{d} I_{\odot}}{\mathrm{d} \tau}\left(\tau, \mu,-\mu_{0}, \varphi-\varphi_{0}\right)=I_{\odot}\left(\tau, \mu,-\mu_{0}, \varphi-\varphi_{0}\right)$.

Considering the Fourier cosine expansions for the phase function

$p\left(\mu, \mu^{\prime}, \varphi-\varphi^{\prime}\right)=\sum_{m=0}^{M_{\max }}\left(2-\delta_{m 0}\right) p_{m}\left(\mu, \mu^{\prime}\right) \cos \left[m\left(\varphi-\varphi^{\prime}\right)\right]$,

and the diffuse radiance

$I_{\mathrm{d}}\left(\tau, \mu,-\mu_{0}, \varphi-\varphi_{0}\right)=\sum_{m=0}^{M_{\max }} I_{\mathrm{d} m}\left(\tau, \mu,-\mu_{0}\right) \cos \left[m\left(\varphi-\varphi_{0}\right)\right]$,

where $M_{\max }$ is the number of azimuthal modes in the expansions, yields the following radiative transfer equation for the individual azimuthal components of the radiance:

$$
\begin{aligned}
\mu \frac{\mathrm{d} I_{\mathrm{d} m}\left(\tau, \mu,-\mu_{0}\right)}{\mathrm{d} \tau} & =I_{\mathrm{d} m}\left(\tau, \mu,-\mu_{0}\right) . \\
& -\left(2-\delta_{m 0}\right) \frac{\omega}{4 \pi} F_{0} p_{m}\left(\mu,-\mu_{0}\right) \mathrm{e}^{-\tau / \mu_{0}} \\
& -\frac{1}{2} \omega \int_{-1}^{1} p_{m}\left(\mu, \mu^{\prime}\right) I_{\mathrm{d} m}\left(\tau, \mu^{\prime},-\mu_{0}\right) \mathrm{d} \mu^{\prime}
\end{aligned}
$$

To simplify notations, hereafter the index $m$ will be suppressed with the dependence on azimuthal mode assumed.

In order to deal with (4) we replace the integral by a symmetric quadrature rule with $2 N$ nodes and weights, i.e., if $\mu_{k}$ with $k=1, \ldots, N$, is a node associated with the weight $w_{k}$, then $-\mu_{k}$ is also a node associated with the same weight. Usually, the quadrature is chosen to be (double) Gaussian, in which case the number of azimuthal modes is $M_{\max } \leq 2 N-1$. This result is discussed in Appendix 1. In the discrete ordinate space, the radiative transfer equation for the diffuse radiance vector $\mathbf{i}_{\mathrm{d}}=\left[\mathbf{i}_{\mathrm{d}}^{+}, \mathbf{i}_{\mathrm{d}}^{-}\right]^{T}$ with $\mathbf{i}_{\mathrm{d}}^{ \pm}=\left[I_{\mathrm{d} m}\left( \pm \mu_{k},-\mu_{0}\right)\right], k=1, \ldots, N$, reads as

$\frac{\mathrm{d} \mathbf{i}_{\mathrm{d}}}{\mathrm{d} \tau}(\tau)=-\mathbf{A i}_{\mathrm{d}}(\tau)-\mathrm{e}^{-\tau / \mu_{0}} \mathbf{b}, 0 \leq \tau \leq \bar{\tau}$.

The entries of the layer matrix

$\mathbf{A}=\left[\begin{array}{cc}\mathbf{A}_{11} & \mathbf{A}_{12} \\ -\mathbf{A}_{12} & -\mathbf{A}_{11}\end{array}\right]$

are

$$
\begin{aligned}
& \mathbf{A}_{11}=\mathbf{M S}+\mathbf{W}-\mathbf{M}, \\
& \mathbf{A}_{12}=\mathbf{M S} \_\mathbf{W},
\end{aligned}
$$

while the entries of the layer vector

$$
\mathbf{b}=\left[\begin{array}{l}
\mathbf{b}_{1} \\
\mathbf{b}_{2}
\end{array}\right]
$$

are

$\mathbf{b}_{1}=\mathbf{M b}_{+}$,

$\mathbf{b}_{2}=-\mathbf{M b}_{-}$,

where

$$
\begin{aligned}
{\left[\mathbf{S}_{ \pm}\right]_{k l} } & =\frac{1}{2} \omega p_{m}\left(\mu_{k}, \pm \mu_{l}\right), \\
{[\mathbf{W}]_{k l} } & =w_{k} \delta_{k l} \\
{[\mathbf{M}]_{k l} } & =\frac{1}{\mu_{k}} \delta_{k l} \\
{\left[\mathbf{b}_{ \pm}\right]_{k} } & =\left(2-\delta_{m 0}\right) \frac{F_{0}}{4 \pi} \omega p_{m}\left( \pm \mu_{k},-\mu_{0}\right),
\end{aligned}
$$

for $k, l=1, \ldots, N$. Here, $\delta_{k l}$ is the Kronecker symbol.

In order to reduce the eigenvalue decomposition of $\mathbf{A}$ from a general to a symmetric problem, we define the scaled diffuse radiance vector $\widehat{\mathbf{i}}_{\mathrm{d}}=\left[\widehat{\mathbf{i}}_{\mathrm{d}}^{+}, \widehat{\mathbf{i}}_{\mathrm{d}}^{-}\right]^{T}$, through the relation

$\widehat{\mathbf{i}}_{\mathrm{d}}^{ \pm}=\mathbf{W}^{\frac{1}{2}} \mathbf{M}^{-\frac{1}{2}} \mathbf{i}_{\mathrm{d}}^{ \pm}$.

Hereafter, the "hat" symbol on vectors and matrices refers to scaled quantities. For the scaled diffuse radiance vector, the radiative transfer equation is

$\frac{d \widehat{\mathbf{i}}_{\mathrm{d}}}{\mathrm{d} \tau}(\tau)=-\widehat{\mathbf{A}} \widehat{\mathbf{i}}_{\mathrm{d}}(\tau)-\mathrm{e}^{-\tau / \mu_{0}} \widehat{\mathbf{b}}, 0 \leq \tau \leq \bar{\tau}$,

where the expressions of the scaled layer matrix $\widehat{\mathbf{A}}$ and the layer vector $\widehat{\mathbf{b}}$ are as in (6) and (8), respectively, with

$\widehat{\mathbf{A}}_{11}=\mathbf{M}^{\frac{1}{2}} \mathbf{W}^{\frac{1}{2}}\left(\mathbf{S}_{+}-\mathbf{W}^{-1}\right) \mathbf{M}^{\frac{1}{2}} \mathbf{W}^{\frac{1}{2}}$,

$\widehat{\mathbf{A}}_{12}=\mathbf{M}^{\frac{1}{2}} \mathbf{W}^{\frac{1}{2}} \mathbf{S}_{-} \mathbf{M}^{\frac{1}{2}} \mathbf{W}^{\frac{1}{2}}$,

and

$\widehat{\mathbf{b}}_{1}=\mathbf{M}^{\frac{1}{2}} \mathbf{W}^{\frac{1}{2}} \mathbf{b}_{+}$,

$\widehat{\mathbf{b}}_{2}=-\mathbf{M}^{\frac{1}{2}} \mathbf{W}^{\frac{1}{2}} \mathbf{b}_{-}$.

From the principle of reciprocity of the phase function, it follows that $\mathbf{S}_{+}$and $\mathbf{S}_{-}$, and so, that $\widehat{\mathbf{A}}_{11}$ and $\widehat{\mathbf{A}}_{12}$ are symmetric matrices. The scaling procedure (14), which is equivalent to the application of a similarity transformation to $\mathbf{A}$ with the diagonal block matrices $\mathbf{W}^{\frac{1}{2}} \mathbf{M}^{-\frac{1}{2}}$, is standard in radiative transfer and has been used by Waterman [21], Nakajima and Tanaka [18], and Stamnes and Swanson [14].

In the framework of the matrix exponential approach, the solution of the initial value problem consisting in the vector differential equation (15) and the initial condition $\widehat{\mathbf{i}}_{\mathrm{d}}(0)$, is given by

$\widehat{\mathbf{i}}_{\mathrm{d}}(\tau)=\mathrm{e}^{-\widehat{\mathbf{A}} \tau} \widehat{\mathbf{i}}_{\mathrm{d}}(0)-\int_{0}^{\tau} \mathrm{e}^{-\widehat{\mathbf{A}}\left(\tau-\tau^{\prime}\right)} \mathrm{e}^{-\tau^{\prime} / \mu_{0}} \widehat{\mathbf{b}} \mathrm{d} \tau^{\prime}$.

Let us give an interpretation of the matrix exponential solution (18). Making use of a spectral decomposition of the matrix $\widehat{\mathbf{A}}$, it can be shown that

$\int_{0}^{\tau} \mathrm{e}^{-\widehat{\mathbf{A}}\left(\tau-\tau^{\prime}\right)} \mathrm{e}^{-\tau^{\prime} / \mu_{0}} \widehat{\mathbf{b}} \mathrm{d} \tau^{\prime}=\left(\mathrm{e}^{-\widehat{\mathbf{A}} \tau}-\mathrm{e}^{-\tau / \mu_{0}} \mathbf{I}\right) \mu_{0}\left(\mathbf{I}-\mu_{0} \widehat{\mathbf{A}}\right)^{-1} \widehat{\mathbf{b}}$, 
whence, setting

$\widehat{\mathbf{c}}=\mu_{0}\left(\mathbf{I}-\mu_{0} \widehat{\mathbf{A}}\right)^{-1} \widehat{\mathbf{b}}$,

we express (18) as

$\left.\widehat{\mathbf{i}}_{\mathrm{d}}(\tau)=\mathrm{e}^{-\widehat{\mathbf{A}} \tau} \widehat{\mathbf{i}}_{\mathrm{d}}(0)-\widehat{\mathbf{c}}\right]+\mathrm{e}^{-\tau / \mu_{0}} \widehat{\mathbf{c}}$.

The classical approach for solving the vector differential equation (15) is to express the general solution as the sum of a homogeneous and a particular solution, i.e.,

$\widehat{\mathbf{i}}_{\mathrm{d}}(\tau)=\widehat{\mathbf{i}}_{\mathrm{h}}(\tau)+\widehat{\mathbf{i}}_{\mathrm{p}}(\tau)$.

The particular integral solving (15), is

$\widehat{\mathbf{i}}_{\mathrm{p}}(\tau)=\mathrm{e}^{-\tau / \mu_{0}} \widehat{\mathbf{c}}$,

where $\widehat{\mathbf{c}}$ is given by (20). The homogeneous or the fundamental solution solves the equation

$\frac{\mathrm{d} \widehat{\mathbf{i}_{\mathrm{h}}}}{\mathrm{d} \tau}(\tau)=-\widehat{\mathbf{A}} \widehat{\mathbf{i}}_{\mathrm{h}}(\tau)$,

and is given by

$\widehat{\mathbf{i}}_{\mathrm{h}}(\tau)=\mathrm{e}^{-\widehat{\mathbf{A}} \tau} \widehat{\mathbf{c}}_{\mathrm{h}}$.

The integration vector $\widehat{\mathbf{c}}_{\mathrm{h}}$ is obtained from (22) and the initial condition $\widehat{\mathbf{i}}_{\mathrm{d}}(0)$; the result is $\widehat{\mathbf{c}}_{\mathrm{h}}=\widehat{\mathbf{i}}_{\mathrm{d}}(0)-\widehat{\mathbf{c}}$. Then, accounting of (22), (23), and (25) we find that an equivalent representation of (21) is

$\widehat{\mathbf{i}}_{\mathrm{d}}(\tau)=\mathrm{e}^{-\widehat{\mathbf{A}} \tau}\left[\widehat{\mathbf{i}}_{\mathrm{d}}(0)-\widehat{\mathbf{i}}_{\mathrm{p}}(0)\right]+\widehat{\mathbf{i}}_{\mathrm{p}}(\tau)$.

If the incident direction $\mu_{0}$ coincides with a discrete ordinate direction, say $\mu_{0}=\mu_{l}$ for some $l$, then the homogeneous and particular solutions can be interpreted as the total and direct radiance vectors, respectively. To show this, let us define the vector $\widehat{\mathbf{i}}_{\odot}(0)=\left[\widehat{\mathbf{i}}_{\odot}^{+}(0), \widehat{\mathbf{i}}_{\odot}^{-}(0)\right]^{T}$ by $\widehat{\mathbf{i}}_{\odot}^{+}(0)=0$ and

$\left.\widehat{\mathbf{i}}_{\odot}^{-}(0)\right]_{k}=\left(2-\delta_{m 0}\right) \frac{F_{0}}{2 \pi} \sqrt{\frac{\mu_{k}}{w_{k}}} \delta_{k l}, k=1, \ldots, N$.

By straightforward calculation it can be shown that $\widehat{\mathbf{i}}_{\odot}(0)=$ $-\widehat{\mathbf{c}}$. As a result, the particular solution $\widehat{\mathbf{i}}_{\mathrm{p}}(\tau)$ given by (23) can be identified with the direct radiance vector $\widehat{\mathbf{i}}_{\odot}(\tau)$, defined by $\widehat{\mathbf{i}}_{\odot}(\tau)=\mathrm{e}^{-\tau / \mu_{0}} \widehat{\mathbf{i}}_{\odot}(0)$, i.e., $\widehat{\mathbf{i}}_{\odot}(\tau)=-\widehat{\mathbf{i}}_{\mathrm{p}}(\tau)$, and so, the total radiance vector $\widehat{\mathbf{i}}(\tau)$, defined by $\widehat{\mathbf{i}}(\tau)=\widehat{\mathbf{i}}_{\mathrm{d}}(\tau)+\widehat{\mathbf{i}}_{\odot}(\tau)$, can be identified with the homogeneous solution $\hat{\mathbf{i}}_{\mathrm{h}}(\tau)$. Note that both $\hat{\mathbf{i}}_{\odot}$ and $\widehat{\mathbf{i}}_{\mathrm{p}}$ solve the differential equation $\mu_{0} \mathrm{~d} \widehat{\mathbf{i}}_{\odot}(\tau) / \mathrm{d} \tau=-\widehat{\mathbf{i}}_{\odot}(\tau)$, while both $\widehat{\mathbf{i}}$ and $\hat{\mathbf{i}}_{\text {h }}$ solve the differential equation (24). It should be pointed out that in a continuous setting, the total radiance is a generalized function, or a distribution, while in a discrete setting and under the above assumption, the total radiance, regarded as a function $\mu_{k}$, has a jump at $\mu_{k}=\mu_{l}$ (= $\left.\mu_{0}\right)$.

In [22], the matrix exponential $\exp (-\widehat{\mathbf{A}} \tau)$, reflecting the internal properties of the homogeneous medium, is called propagator and is denoted by $\mathbf{P}(\tau)$, i.e., $\mathbf{P}(\tau)=\exp (-\widehat{\mathbf{A}} \tau)$. If the initial condition is given, then the solution deeper in the medium can be recovered (propagated) down from the upper boundary by applying this propagator. However, the obtained solution has no physical meaning, as long as the radiative transfer equation cannot be treated as an initial value problem. The initial condition means that both sets of upward and downward radiances at the upper boundary are known, a fact which typically does not occur in atmospheric radiative transfer. This by no means reduces the usefulness of matrix exponential (propagator), as it will be demonstrated in the course of our analysis.

\section{Eigendecomposition method for computing the matrix exponential}

The matrix exponential can be computed by using an eigendecomposition of the matrix $\widehat{\mathbf{A}}$. Exploiting the block symmetry of $\widehat{\mathbf{A}}$, we find

$\widehat{\mathbf{A}}=\widehat{\mathbf{V}}\left[\begin{array}{cc}\boldsymbol{\Lambda} & \mathbf{0} \\ \mathbf{0} & -\boldsymbol{\Lambda}\end{array}\right] \widehat{\mathbf{V}}^{-1}$,

with

$\widehat{\mathbf{V}}=\left[\begin{array}{ll}\widehat{\mathbf{V}}_{+} & \widehat{\mathbf{V}}_{-} \\ \widehat{\mathbf{V}}_{-} & \widehat{\mathbf{V}}_{+}\end{array}\right]$

and (the abbreviation 'not' stands for notation)

$\Lambda=\operatorname{diag}\left[\lambda_{1}, \ldots, \lambda_{N}\right] \stackrel{\text { not }}{=}\left[\lambda_{k}\right]$.

The spectral decomposition of the matrix $\widehat{\mathbf{A}}$ can be obtained by one of the following methods: direct decomposition of an asymmetric matrix [14], square-root decomposition [18], and Cholesky decomposition [30]. These approaches are summarized in Appendix 2. In (28), the matrices $\widehat{\mathbf{V}}_{ \pm}$are of the form

$\widehat{\mathbf{V}}_{ \pm}=\left[\widehat{\mathbf{v}}_{1}^{ \pm}, \ldots, \widehat{\mathbf{v}}_{N}^{ \pm}\right] \stackrel{\text { not }}{=}\left[\widehat{\mathbf{v}}_{k}^{ \pm}\right]$,

where $\left[\begin{array}{c}\widehat{\mathbf{v}}_{k}^{+} \\ \widehat{\mathbf{v}}_{k}^{-}\end{array}\right]$are the right eigenvectors of $\widehat{\mathbf{A}}$ corresponding to $\lambda_{k}$, and $\left[\begin{array}{c}\widehat{\mathbf{v}}_{k}^{-} \\ \widehat{\mathbf{v}}_{k}^{+}\end{array}\right]$are the right eigenvectors of $\widehat{\mathbf{A}}$ corresponding to $-\lambda_{k}$. The matrix exponential is then given by

$\mathrm{e}^{-\widehat{\mathbf{A}} \tau}=\widehat{\mathbf{V}}\left[\begin{array}{cc}\boldsymbol{\Gamma}(\tau) & \mathbf{0} \\ \mathbf{0} & \boldsymbol{\Gamma}(-\tau)\end{array}\right] \widehat{\mathbf{V}}^{-1}$

with

$\boldsymbol{\Gamma}(\tau)=\left[\mathrm{e}^{-\lambda_{k} \tau}\right]$.

From (30) it is apparent that the computation of the matrix exponential requires the computation of the inverse of the right eigenvectors matrix $\widehat{\mathbf{V}}$. In Waterman's approach, the inverse $\widehat{\mathbf{V}}^{-1}$ is computed by using the following result: For any matrix A, which has a complete set of linearly independent eigenvectors, the inverse of the right eigenvector matrix is the transpose of the left eigenvector matrix. Indeed, let $\mathbf{A}$ be an 
$n \times n$ matrix with distinct eigenvalues, $\mathbf{x}_{k}$ be a right eigenvector of $\mathbf{A}$ corresponding to $\lambda_{k}$, i.e., $\mathbf{A} \mathbf{x}_{k}=\lambda_{k} \mathbf{x}_{k}$, and $\mathbf{y}_{l}$ be a left eigenvector of $\mathbf{A}$ corresponding to $\lambda_{l}$, i.e., $\mathbf{A}^{T} \mathbf{y}_{l}=\lambda_{l} \mathbf{y}_{l}$. Then, from $\mathbf{y}_{l}^{T} \mathbf{A} \mathbf{x}_{k}=\lambda_{k} \mathbf{y}_{l}^{T} \mathbf{x}_{k}=\lambda_{l} \mathbf{y}_{l}^{T} \mathbf{x}_{k}$, we see that for $\lambda_{k} \neq \lambda_{l}$, we have $\mathbf{y}_{l}^{T} \mathbf{x}_{k}=0$. Moreover, assuming that $\mathbf{x}_{k}$ and $\mathbf{y}_{k}$ are normalized in the sense that $\mathbf{y}_{k}^{T} \mathbf{x}_{k}=1, k=1, \ldots, n$, we find that $\mathbf{X}^{-1}=\mathbf{Y}^{T}$, where $\mathbf{X}=\left[\mathbf{x}_{k}\right]$ and $\mathbf{Y}=\left[\mathbf{y}_{k}\right]$. Thus, the spectral decomposition of $\mathbf{A}$ reads as $\mathbf{A}=\mathbf{X} \mathbf{\Lambda} \mathbf{X}^{-1}=\mathbf{X} \mathbf{\Lambda} \mathbf{Y}^{T}$.

Accounting of (27)-(29), we deduce that the systems of normalized right and left eigenvectors corresponding to $\lambda_{k}$ and $-\lambda_{k}$ are

$\left(\lambda_{k}, \frac{1}{\sqrt{\left|a_{k}\right|}}\left[\begin{array}{c}\widehat{\mathbf{v}}_{k}^{+} \\ \widehat{\mathbf{v}}_{k}^{-}\end{array}\right], \frac{\operatorname{sign}\left(a_{k}\right)}{\sqrt{\left|a_{k}\right|}}\left[\begin{array}{c}-\widehat{\mathbf{v}}_{k}^{+} \\ \widehat{\mathbf{v}}_{k}^{-}\end{array}\right]\right)$

and

$\left(-\lambda_{k}, \frac{1}{\sqrt{\left|a_{k}\right|}}\left[\begin{array}{c}\widehat{\mathbf{v}}_{k}^{-} \\ \widehat{\mathbf{v}}_{k}^{+}\end{array}\right], \frac{\operatorname{sign}\left(a_{k}\right)}{\sqrt{\left|a_{k}\right|}}\left[\begin{array}{c}\widehat{\mathbf{v}}_{k}^{-} \\ -\widehat{\mathbf{v}}_{k}^{+}\end{array}\right]\right)$,

respectively, with $a_{k}=\left\|\widehat{\mathbf{v}}_{k}^{-}\right\|^{2}-\left\|\widehat{\mathbf{v}}_{k}^{+}\right\|^{2}$. To simplify notations we put

$\frac{1}{\sqrt{\left|a_{k}\right|}} \widehat{\mathbf{v}}_{k}^{ \pm} \rightarrow \widehat{\mathbf{v}}_{k}^{ \pm}$and $\overline{\mathbf{v}}_{k}^{ \pm}=\operatorname{sign}\left(a_{k}\right) \widehat{\mathbf{v}}_{k}^{ \pm}$

so that

$\left(\lambda_{k},\left[\begin{array}{c}\widehat{\mathbf{v}}_{k}^{+} \\ \widehat{\mathbf{v}}_{k}^{-}\end{array}\right],\left[\begin{array}{c}-\overline{\mathbf{v}}_{k}^{+} \\ \overline{\mathbf{v}}_{k}^{-}\end{array}\right]\right)$

and

$\left(-\lambda_{k},\left[\begin{array}{c}\widehat{\mathbf{v}}_{k}^{-} \\ \widehat{\mathbf{v}}_{k}^{+}\end{array}\right],\left[\begin{array}{c}\overline{\mathbf{v}}_{k}^{-} \\ -\overline{\mathbf{v}}_{k}^{+}\end{array}\right]\right)$

are the systems of normalized right and left eigenvectors corresponding to $\lambda_{k}$ and $-\lambda_{k}$, respectively. Thus, we have $\widehat{\mathbf{V}}^{-1}=$ $\overline{\mathbf{V}}^{T}$, with

$$
\overline{\mathbf{V}}=\left[\begin{array}{cc}
-\overline{\mathbf{V}}_{+} & \overline{\mathbf{V}}_{-} \\
\overline{\mathbf{V}}_{-} & -\overline{\mathbf{V}}_{+}
\end{array}\right]
$$

$\overline{\mathbf{V}}_{ \pm}=\left[\overline{\mathbf{v}}_{k}^{ \pm}\right]$.

The spectral decomposition of $\widehat{\mathbf{A}}$ is then

$\widehat{\mathbf{A}}=\widehat{\mathbf{V}}\left[\begin{array}{cc}\boldsymbol{\Lambda} & \mathbf{0} \\ \mathbf{0} & -\boldsymbol{\Lambda}\end{array}\right] \overline{\mathbf{V}}^{T}$,

or explicitly,

$\widehat{\mathbf{A}}=\sum_{k=1}^{N} \lambda_{k}\left[\begin{array}{c}\widehat{\mathbf{v}}_{k}^{+} \\ \widehat{\mathbf{v}}_{k}^{-}\end{array}\right]\left[\begin{array}{c}-\overline{\mathbf{v}}_{k}^{+} \\ \overline{\mathbf{v}}_{k}^{-}\end{array}\right]^{T}-\lambda_{k}\left[\begin{array}{c}\widehat{\mathbf{v}}_{k}^{-} \\ \widehat{\mathbf{v}}_{k}^{+}\end{array}\right]\left[\begin{array}{c}\overline{\mathbf{v}}_{k}^{-} \\ -\overline{\mathbf{v}}_{k}^{+}\end{array}\right]^{T}$,

while the matrix exponential is

$\mathrm{e}^{-\widehat{\mathbf{A}} \tau}=\widehat{\mathbf{V}}\left[\begin{array}{cc}\boldsymbol{\Gamma}(\tau) & \mathbf{0} \\ \mathbf{0} & \boldsymbol{\Gamma}(-\tau)\end{array}\right] \overline{\mathbf{V}}^{T}$ or explicitly,

$\mathrm{e}^{-\widehat{\mathbf{A}} \tau}=\sum_{k=1}^{N} \mathrm{e}^{-\lambda_{k} \tau}\left[\begin{array}{c}\widehat{\mathbf{v}}_{k}^{+} \\ \widehat{\mathbf{v}}_{k}^{-}\end{array}\right]\left[\begin{array}{c}-\overline{\mathbf{v}}_{k}^{+} \\ \overline{\mathbf{v}}_{k}^{-}\end{array}\right]^{T}+\mathrm{e}^{\lambda_{k} \tau}\left[\begin{array}{c}\widehat{\mathbf{v}}_{k}^{-} \\ \widehat{\mathbf{v}}_{k}^{+}\end{array}\right]\left[\begin{array}{c}\overline{\mathbf{v}}_{k}^{-} \\ -\overline{\mathbf{v}}_{k}^{+}\end{array}\right]^{T}$.

A short comment is in order. In the absence of scattering $(\omega=0)$, we have $\widehat{\mathbf{A}}_{11}=-\widehat{\mathbf{A}}_{22}=-\mathbf{M}$, and $\widehat{\mathbf{A}}_{12}=\widehat{\mathbf{A}}_{21}=0$. As a result, we obtain $\widehat{\mathbf{V}}_{+}=0, \widehat{\mathbf{V}}_{-}=\mathbf{I}$, and $\boldsymbol{\Lambda}=\mathbf{M}$; thus, the eigenvalues are the inverse of the discrete ordinates, i.e., $\lambda_{k}=1 / \mu_{k}$. The matrix exponential is the diagonal matrix $\exp (\widehat{\mathbf{A}} \tau)=\left[\mathrm{e}^{\lambda_{k} \tau} ; \mathrm{e}^{-\lambda_{k} \tau}\right]$ and the homogeneous solution at $\tau=$ $\bar{\tau}$, given by $\widehat{\mathbf{i}}_{\mathrm{h}}(\bar{\tau})=\left[\mathrm{e}^{\lambda_{k} \bar{\tau}} ; \mathrm{e}^{-\lambda_{k} \bar{\tau}}\right] \widehat{\mathbf{i}}_{\mathrm{h}}(0)$, is a representation of the Beer-Lambert attenuation law for the downward and upward radiances, i.e., $\left[\widehat{\mathbf{i}}_{\mathrm{h}}^{-}(\bar{\tau})\right]_{k}=\exp \left(-\lambda_{k} \bar{\tau}\right)\left[\widehat{\mathbf{i}}_{\mathrm{h}}^{-}(0)\right]_{k}$ and $\left[\widehat{\mathbf{i}}_{\mathrm{h}}^{+}(0)\right]_{k}=$ $\exp \left(-\lambda_{k} \bar{\tau}\right)\left[\hat{\mathbf{i}}_{\mathrm{h}}^{+}(\bar{\tau})\right]_{k}$, respectively. If scattering is present, the Beer-Lambert law is still valid but for the downward and upward radiances $\xi_{-}(\bar{\tau})$ and $\xi_{-}(0)$ corresponding to the transformed radiance vector

$\boldsymbol{\xi}(\tau)=\left[\begin{array}{c}\boldsymbol{\xi}_{+}(\tau) \\ \boldsymbol{\xi}_{-}(\tau)\end{array}\right]=\left[\begin{array}{ll}0 & \mathbf{I} \\ \mathbf{I} & 0\end{array}\right] \overline{\mathbf{V}}^{T} \widehat{\mathbf{i}}_{\mathrm{h}}(\tau)$.

We proceed now to derive some matrix identities which will be frequently used in the following. In terms of block matrices, the orthogonality relation $\widehat{\mathbf{V}} \overline{\mathbf{V}}^{T}=\mathbf{I}_{2 N}$, where $\mathbf{I}_{2 N}$ is the identity matrix of dimension $2 N \times 2 N$, reads as

$$
\left[\begin{array}{cc}
\widehat{\mathbf{V}}_{+} & \widehat{\mathbf{V}}_{-} \\
\widehat{\mathbf{V}}_{-} & \widehat{\mathbf{V}}_{+}
\end{array}\right]\left[\begin{array}{cc}
-\overline{\mathbf{V}}_{+} & \overline{\mathbf{V}}_{-} \\
\overline{\mathbf{V}}_{-} & -\overline{\mathbf{V}}_{+}
\end{array}\right]^{T}=\mathbf{I}_{2 N}
$$

from which we infer that

$\widehat{\mathbf{V}}_{-} \overline{\mathbf{V}}_{-}^{T}-\widehat{\mathbf{V}}_{+} \overline{\mathbf{V}}_{+}^{T}=\mathbf{I}_{N}$

$$
\widehat{\mathbf{V}}_{+} \overline{\mathbf{V}}_{-}^{T}=\widehat{\mathbf{V}}_{-} \overline{\mathbf{V}}_{+}^{T} \text {. }
$$

Similarly, from $\overline{\mathbf{V}}^{T} \widehat{\mathbf{V}}=\mathbf{I}_{2 N}$, we obtain

$$
\begin{aligned}
\overline{\mathbf{V}}_{-}^{T} \widehat{\mathbf{V}}_{-}-\overline{\mathbf{V}}_{+}^{T} \widehat{\mathbf{V}}_{+} & =\mathbf{I}_{N}, \\
\overline{\mathbf{V}}_{-}^{T} \widehat{\mathbf{V}}_{+} & =\overline{\mathbf{V}}_{+}^{T} \widehat{\mathbf{V}}_{-} .
\end{aligned}
$$

Accounting of (44)-(47), the following matrix identities readily follow:

$$
\begin{aligned}
\overline{\mathbf{V}}_{+}^{T} \overline{\mathbf{V}}_{-}^{-T} & =\widehat{\mathbf{V}}_{-}^{-1} \widehat{\mathbf{V}}_{+}, \\
\overline{\mathbf{V}}_{-}^{-T} \overline{\mathbf{V}}_{+}^{T} & =\widehat{\mathbf{V}}_{+} \widehat{\mathbf{V}}_{-}^{-1}, \\
\overline{\mathbf{V}}_{-}^{-T} & =\widehat{\mathbf{V}}_{-}-\widehat{\mathbf{V}}_{+} \widehat{\mathbf{V}}_{-}^{-1} \widehat{\mathbf{V}}_{+} .
\end{aligned}
$$

On the other hand, from (34) and (38), we see that

$\overline{\mathbf{V}}_{ \pm}=\widehat{\mathbf{V}}_{ \pm} \mathbb{S}$

where $\mathbb{S}=\left[\operatorname{sign}\left(a_{k}\right)\right]$ is a diagonal matrix of plus and minus ones. As $\mathbb{S} S=\mathbf{I}_{N}$, we find that the matrices $\widehat{\mathbf{V}}_{+}^{T} \widehat{\mathbf{V}}_{-}$and $\widehat{\mathbf{V}}_{+} \widehat{\mathbf{V}}_{-}^{-1}$ are symmetric, i.e.,

$$
\begin{aligned}
\left(\widehat{\mathbf{V}}_{+}^{T} \widehat{\mathbf{V}}_{-}\right)^{T} & =\widehat{\mathbf{V}}_{+}^{T} \widehat{\mathbf{V}}_{-}, \\
\left(\widehat{\mathbf{V}}_{+} \widehat{\mathbf{V}}_{-}^{-1}\right)^{T} & =\widehat{\mathbf{V}}_{+} \widehat{\mathbf{V}}_{-}^{-1},
\end{aligned}
$$


but $\widehat{\mathbf{V}}_{-} \widehat{\mathbf{V}}_{+}^{T}$ and $\widehat{\mathbf{V}}_{-}^{-1} \widehat{\mathbf{V}}_{+}$are not; in particular, we have

$$
\begin{aligned}
\left(\widehat{\mathbf{V}}_{-} \mathbb{S} \widehat{\mathbf{V}}_{+}^{T}\right)^{T} & =\widehat{\mathbf{V}}_{-} \mathbb{S} \widehat{\mathbf{V}}_{+}^{T}, \\
\left(\widehat{\mathbf{V}}_{-}^{-1} \widehat{\mathbf{V}}_{+}\right)^{T} & =\mathbb{S}\left(\widehat{\mathbf{V}}_{-}^{-1} \widehat{\mathbf{V}}_{+}\right) \mathbb{S} .
\end{aligned}
$$

Solution (18) with the matrix exponential as in (41) is the starting point in our analysis. This solution, in the form of the propagator $\mathbf{P}(\tau)=\exp (-\widehat{\mathbf{A}} \tau)$, is a combination of both growing and decaying exponentials. For large optical thicknesses, growing exponentials will dominate the solution given in this form, and the direct application of the propagator leads to numerical instability. This behavior is referred to as dichotomic [22]. However, by appropriate manipulations of (18) and by introducing scaling transformations, computationally stable equations, the so-called layer equation and the interaction principle equation can be derived. These equations, which are the quintessence of the discrete ordinate and matrix operator method with matrix exponential, are discussed in Sections 4 and 5, respectively.

\section{Discrete ordinate method with matrix exponential}

In the framework of the discrete ordinate method with matrix exponential, the layer equation is a computationally stable relation connecting the layer-top radiance vector $\widehat{\mathbf{i}}_{\mathrm{d}}(0)$ and the layer-bottom radiance vector $\widehat{\mathbf{i}}_{\mathrm{d}}(\tau)[25,26]$. For a multi-layered atmosphere, each layer equation is assembled into the system matrix of the entire atmosphere. By imposing appropriate boundary conditions at the top and the bottom of the atmosphere, the system of equations is solved for the level values of the radiances. Thus, the method avoids computing an explicit solution for each layer by imposing boundary conditions for the entire atmosphere, as well as, the continuity condition for the radiances across the layer interfaces.

The layer equation is derived by inserting the matrix exponential representation (41) in (18), and by multiplying the resulting equation with an appropriate scaling matrix as in [31]. The result is

$\mathbf{D}_{1}(\bar{\tau}) \overline{\mathbf{V}}^{T} \widehat{\mathbf{i}}_{\mathrm{d}}(\bar{\tau})=\mathbf{D}_{0}(\bar{\tau}) \overline{\mathbf{V}}^{T} \widehat{\mathbf{i}}_{\mathrm{d}}(0)-\mathbf{D}_{\mathrm{b}}(\bar{\tau}) \overline{\mathbf{V}}^{T} \widehat{\mathbf{b}}$,

where

$$
\begin{aligned}
& \mathbf{D}_{1}(\bar{\tau})=\left[\begin{array}{cc}
\mathbf{I}_{N} & \mathbf{0} \\
\mathbf{0} & \boldsymbol{\Gamma}(\bar{\tau})
\end{array}\right], \mathbf{D}_{0}(\bar{\tau})=\left[\begin{array}{cc}
\boldsymbol{\Gamma}(\bar{\tau}) & \mathbf{0} \\
\mathbf{0} & \mathbf{I}_{N}
\end{array}\right], \\
& \mathbf{D}_{\mathrm{b}}(\bar{\tau})=\left[\begin{array}{cc}
\frac{\mathrm{e}^{-\lambda} k^{\bar{\tau}}-\mathrm{e}^{-\bar{\tau} / \mu_{0}}}{1 / \mu_{0}-\lambda_{k}} & \mathbf{0} \\
\mathbf{0} & \frac{1-\mathrm{e}^{-\bar{\tau}\left(\lambda_{k}+1 / \mu_{0}\right)}}{1 / \mu_{0}+\lambda_{k}}
\end{array}\right] .
\end{aligned}
$$

If the level values of the radiances $\widehat{\mathbf{i}}_{\mathrm{d}}(0)$ and $\widehat{\mathbf{i}}_{\mathrm{d}}(\bar{\tau})$ are known, the radiance at an internal point $\tau$, with $0 \leq \tau \leq \bar{\tau}$, is computed as

$$
\widehat{\mathbf{i}}_{\mathrm{d}}(\tau)=\widehat{\mathbf{V}} \mathbf{E}(\tau, \bar{\tau})\left[\begin{array}{c}
\boldsymbol{\xi}_{+}(0) \\
\boldsymbol{\xi}_{-}(\bar{\tau})
\end{array}\right]-\widehat{\mathbf{V}} \mathbf{E}_{\mathrm{b}}(\tau, \bar{\tau}) \boldsymbol{\eta}
$$

where now $\boldsymbol{\xi}(\tau)=\left[\boldsymbol{\xi}_{+}(\tau), \boldsymbol{\xi}_{-}(\tau)\right]^{T}=\overline{\mathbf{V}}^{T} \widehat{\mathbf{i}}_{\mathrm{d}}(\tau), \boldsymbol{\eta}=\overline{\mathbf{V}}^{T} \widehat{\mathbf{b}}$, and

$$
\begin{aligned}
\mathbf{E}(\tau, \bar{\tau}) & =\left[\begin{array}{cc}
\boldsymbol{\Gamma}(\tau) & 0 \\
0 & \boldsymbol{\Gamma}(\bar{\tau}-\tau)
\end{array}\right], \\
\mathbf{E}_{\mathbf{b}}(\tau, \bar{\tau}) & =\left[\begin{array}{cc}
\frac{\mathrm{e}^{-\lambda_{k} \tau}-\mathrm{e}^{-\tau / \mu_{0}}}{1 / \mu_{0}-\lambda_{k}} & \mathbf{0} \\
\mathbf{0} & -\mathrm{e}^{-\tau / \mu_{0}} \frac{1-\mathrm{e}^{-(\bar{\tau}-\tau)\left(\lambda_{k}+1 / \mu_{0}\right)}}{1 / \mu_{0}+\lambda_{k}}
\end{array}\right] .
\end{aligned}
$$

The matrix exponential representation of the solution as given by (18) is mathematically equivalent to the classical Chandrasekhar's representation in terms of the characteristic solutions

$\mathrm{e}^{-\lambda_{k} \tau}\left[\begin{array}{c}\widehat{\mathbf{v}}_{k}^{+} \\ \widehat{\mathbf{v}}_{k}^{-}\end{array}\right]$and $\mathrm{e}^{\lambda_{k} \tau}\left[\begin{array}{c}\widehat{\mathbf{v}}_{k}^{-} \\ \widehat{\mathbf{v}}_{k}^{+}\end{array}\right]$

To show this equivalences, we consider (20), i.e.,

$\left.\widehat{\mathbf{i}}_{\mathrm{d}}(\tau)=\mathrm{e}^{-\widehat{\mathbf{A}} \tau} \widehat{\hat{\mathbf{i}}_{\mathrm{d}}}(0)-\widehat{\mathbf{i}}_{\mathrm{p}}(0)\right]+\widehat{\mathbf{i}}_{\mathrm{p}}(\tau)$,

for $0 \leq \tau \leq \bar{\tau}$. Using (30) and writing

$$
\begin{aligned}
{\left[\begin{array}{cc}
\boldsymbol{\Gamma}(\tau) & \mathbf{0} \\
\mathbf{0} & \boldsymbol{\Gamma}(-\tau)
\end{array}\right] } & =\left[\begin{array}{cc}
\boldsymbol{\Gamma}(\tau) & \mathbf{0} \\
\mathbf{0} & \boldsymbol{\Gamma}(-\tau)
\end{array}\right] \\
& \times\left[\begin{array}{cc}
\mathbf{I} & \mathbf{0} \\
\mathbf{0} & \boldsymbol{\Gamma}(\bar{\tau})
\end{array}\right]\left[\begin{array}{cc}
\mathbf{I} & \mathbf{0} \\
\mathbf{0} & \boldsymbol{\Gamma}(\bar{\tau})
\end{array}\right]^{-1},
\end{aligned}
$$

we obtain

$\widehat{\mathbf{i}}_{\mathrm{d}}(\tau)=\left[\begin{array}{ll}\widehat{\mathbf{V}}_{+} \boldsymbol{\Gamma}(\tau) & \widehat{\mathbf{V}}_{-} \boldsymbol{\Gamma}(\bar{\tau}-\tau) \\ \widehat{\mathbf{V}}_{-} \boldsymbol{\Gamma}(\tau) & \widehat{\mathbf{V}}_{+} \boldsymbol{\Gamma}(\bar{\tau}-\tau)\end{array}\right]\left[\begin{array}{c}\boldsymbol{\alpha} \\ \boldsymbol{\beta}\end{array}\right]+\widehat{\mathbf{i}}_{\mathrm{p}}(\tau)$,

where the $N$-dimensional vectors $\boldsymbol{\alpha}$ and $\boldsymbol{\beta}$ do not depend on $\tau$, and are given by

$\left.\left[\begin{array}{c}\boldsymbol{\alpha} \\ \boldsymbol{\beta}\end{array}\right]=\left[\begin{array}{ll}\widehat{\mathbf{V}}_{+} & \widehat{\mathbf{V}}_{-} \boldsymbol{\Gamma}(\bar{\tau}) \\ \widehat{\mathbf{V}}_{-} & \widehat{\mathbf{V}}_{+} \boldsymbol{\Gamma}(\bar{\tau})\end{array}\right]^{-1} \widehat{\mathbf{i}}_{\mathrm{d}}(0)-\widehat{\mathbf{i}}_{\mathrm{p}}(0)\right]$

The explicit form of (63), i.e.,

$\widehat{\mathbf{i}}_{\mathrm{d}}(\tau)=\sum_{k=1}^{N} \alpha_{k} \mathrm{e}^{-\lambda_{k} \tau}\left[\begin{array}{c}\widehat{\mathbf{v}}_{k}^{+} \\ \widehat{\mathbf{v}}_{k}^{-}\end{array}\right]+\beta_{k} \mathrm{e}^{-\lambda_{k}(\bar{\tau}-\tau)}\left[\begin{array}{c}\widehat{\mathbf{v}}_{k}^{-} \\ \widehat{\mathbf{v}}_{k}^{+}\end{array}\right]+\hat{\mathbf{i}}_{\mathrm{p}}(\tau)$,

is the solution representation in the Chandrasekhar's discrete ordinate method. Another representation can be obtained by using the relation

$$
\begin{aligned}
{\left[\begin{array}{cc}
\boldsymbol{\Gamma}(\tau) & \mathbf{0} \\
\mathbf{0} & \boldsymbol{\Gamma}(-\tau)
\end{array}\right] } & =\left[\begin{array}{cc}
\boldsymbol{\Gamma}(\tau) & \mathbf{0} \\
\mathbf{0} & \boldsymbol{\Gamma}(-\tau)
\end{array}\right] . \\
& \times\left[\begin{array}{cc}
\mathbf{I} & -\mathbf{I} \\
\boldsymbol{\Gamma}(\bar{\tau}) & \boldsymbol{\Gamma}(\bar{\tau})
\end{array}\right]\left[\begin{array}{cc}
\mathbf{I} & -\mathbf{I} \\
\boldsymbol{\Gamma}(\bar{\tau}) & \boldsymbol{\Gamma}(\bar{\tau})
\end{array}\right]^{-1},
\end{aligned}
$$

which yields

$$
\begin{aligned}
\widehat{\mathbf{i}}_{\mathrm{d}}(\tau) & =\left[\begin{array}{ll}
\widehat{\mathbf{V}}_{-} \boldsymbol{\Gamma}(\bar{\tau}-\tau)+\widehat{\mathbf{V}}_{+} \boldsymbol{\Gamma}(\tau) & \widehat{\mathbf{V}}_{-} \boldsymbol{\Gamma}(\bar{\tau}-\tau)-\widehat{\mathbf{V}}_{+} \boldsymbol{\Gamma}(\tau) \\
\widehat{\mathbf{V}}_{+} \boldsymbol{\Gamma}(\bar{\tau}-\tau)+\widehat{\mathbf{V}}_{-} \boldsymbol{\Gamma}(\tau) & \widehat{\mathbf{V}}_{+} \boldsymbol{\Gamma}(\bar{\tau}-\tau)-\widehat{\mathbf{V}}_{-} \boldsymbol{\Gamma}(\tau)
\end{array}\right] \\
& \times\left[\begin{array}{c}
\boldsymbol{\alpha} \\
\boldsymbol{\beta}
\end{array}\right]+\widehat{\mathbf{i}}_{\mathrm{p}}(\tau),
\end{aligned}
$$


with

$$
\left[\begin{array}{c}
\boldsymbol{\alpha} \\
\boldsymbol{\beta}
\end{array}\right]=\left[\begin{array}{ll}
\widehat{\mathbf{V}}_{-} \boldsymbol{\Gamma}(\bar{\tau})+\widehat{\mathbf{V}}_{+} & \widehat{\mathbf{V}}_{-} \boldsymbol{\Gamma}(\bar{\tau})-\widehat{\mathbf{V}}_{+} \\
\widehat{\mathbf{V}}_{+} \boldsymbol{\Gamma}(\bar{\tau})+\widehat{\mathbf{V}}_{-} & \widehat{\mathbf{V}}_{+} \boldsymbol{\Gamma}(\bar{\tau})-\widehat{\mathbf{V}}_{-}
\end{array}\right]^{-1}\left[\widehat{\mathbf{i}}_{\mathrm{d}}(0)-\widehat{\mathbf{i}}_{\mathrm{p}}(0)\right]
$$

From (67), we get

$$
\begin{aligned}
\widehat{\mathbf{i}}_{\mathrm{d}}(\tau) & =\sum_{k=1}^{N} \alpha_{k}\left(\mathrm{e}^{-\lambda_{k} \tau}\left[\begin{array}{c}
\widehat{\mathbf{v}}_{k}^{+} \\
\widehat{\mathbf{v}}_{k}^{-}
\end{array}\right]+\mathrm{e}^{-\lambda_{k}(\bar{\tau}-\tau)}\left[\begin{array}{c}
\widehat{\mathbf{v}}_{k}^{-} \\
\widehat{\mathbf{v}}_{k}^{+}
\end{array}\right]\right) \\
& +\beta_{k}\left(-\mathrm{e}^{-\lambda_{k} \tau}\left[\begin{array}{c}
\widehat{\mathbf{v}}_{k}^{+} \\
\widehat{\mathbf{v}}_{k}^{-}
\end{array}\right]+\mathrm{e}^{-\lambda_{k}(\bar{\tau}-\tau)}\left[\begin{array}{c}
\widehat{\mathbf{v}}_{k}^{-} \\
\widehat{\mathbf{v}}_{k}^{+}
\end{array}\right]\right)+\hat{\mathbf{i}}_{\mathrm{p}}(\tau),
\end{aligned}
$$

where as before, the integration constants $\alpha_{k}$ and $\beta_{k}$ do not depend on $\tau$.

Equations (63)-(64) and (67)-(68) are equivalent solution representations in the matrix exponential method and will be used in the next section to derive the reflection and transmission matrices. In both representations, the radiance vector at optical depth $\tau$ is a superposition of eigenfields propagating from the upper and lower boundaries, with the attenuation factors $\exp \left(-\lambda_{k} \tau\right)$ and $\exp \left[-\lambda_{k}(\bar{\tau}-\tau)\right]$, respectively.

Although the classical and the matrix exponential version of the discrete ordinate method are very similar, several differences can be emphasized:

1. In the classical discrete ordinate method, the expansion coefficients $\alpha_{k}$ and $\beta_{k}$ are the unknowns of the discretized radiative transfer problem and are computed by imposing the continuity condition for the radiances across the layer interfaces. In the discrete ordinate method with matrix exponential, the unknowns are the level values of the radiances. Once they are computed, the integration constants can be obtained from (64) or (68).

2. In the classical discrete ordinate method, the computation of the particular solution requires the computation of the inverse $\left(\mathbf{I}-\mu_{0} \widehat{\mathbf{A}}\right)^{-1}$. This inversion step is not present in the discrete ordinate method with matrix exponential. However, if the systems of normalized right and left eigenvectors stay at our disposal, the constant vector $\widehat{\mathbf{c}}$ which enters in (23), can be calculated with a less computational effort as

$$
\widehat{\mathbf{c}}=\mu_{0} \widehat{\mathbf{V}}\left[\begin{array}{cc}
\left(\mathbf{I}-\mu_{0} \boldsymbol{\Lambda}\right)^{-1} & \mathbf{0} \\
\mathbf{0} & \left(\mathbf{I}+\mu_{0} \boldsymbol{\Lambda}\right)^{-1}
\end{array}\right] \overline{\mathbf{V}}^{T} \widehat{\mathbf{b}}
$$

for $\mu_{0} \neq \lambda_{k}, k=1, \ldots, N$.

\section{Matrix operator method with matrix exponential}

In a continuous setting, the interaction principle equation, which is the central feature of the matrix operator method, relates the outcoming radiances at the layer top $I_{\mathrm{d}}\left(0, \mu,-\mu_{0}, \varphi-\right.$ $\left.\varphi_{0}\right)$ and layer bottom $I_{\mathrm{d}}\left(\bar{\tau},-\mu,-\mu_{0}, \varphi-\varphi_{0}\right)$ to the incoming radiances $I_{\mathrm{d}}\left(0,-\mu,-\mu_{0}, \varphi-\varphi_{0}\right)$ and $I_{\mathrm{d}}\left(\bar{\tau}, \mu,-\mu_{0}, \varphi-\varphi_{0}\right)$ through the reflection, total transmission and diffuse transmission functions $R\left(\mu, \mu^{\prime}, \varphi-\varphi^{\prime} ; \bar{\tau}\right), T\left(\mu, \mu^{\prime}, \varphi-\varphi^{\prime} ; \bar{\tau}\right)$ and $T_{\mathrm{d}}\left(\mu, \mu^{\prime}, \varphi-\right.$ $\left.\varphi^{\prime} ; \bar{\tau}\right)$, respectively, where $\mu, \mu^{\prime}>0$. The transmission function for the diffuse radiance $T_{\mathrm{d}}$ is related to the transmission function for the total radiance by the relation

$$
T_{\mathrm{d}}\left(\mu, \mu^{\prime}, \varphi-\varphi^{\prime} ; \bar{\tau}\right)=T\left(\mu, \mu^{\prime}, \varphi-\varphi^{\prime} ; \bar{\tau}\right)-\frac{\pi}{\mu^{\prime}} \delta\left(\mu-\mu^{\prime}\right) \delta\left(\varphi-\varphi^{\prime}\right) \mathrm{e}^{-\bar{\tau} / \mu^{\prime}}
$$

Considering the Fourier cosine expansions

$$
\begin{aligned}
I_{\mathrm{d}}\left(\bar{\tau}, \mu,-\mu_{0}, \varphi-\varphi_{0}\right) & =\sum_{m=0}^{M_{\max }} I_{\mathrm{d} m}\left(\bar{\tau}, \mu,-\mu_{0}\right) \cos \left[m\left(\varphi-\varphi_{0}\right)\right], \quad \text { (72) } \\
X\left(\mu, \mu^{\prime}, \varphi-\varphi^{\prime} ; \bar{\tau}\right) & =\sum_{m=0}^{M_{\max }}\left(2-\delta_{m 0}\right) X_{m}\left(\mu, \mu^{\prime} ; \bar{\tau}\right) \cos \left[m\left(\varphi-\varphi^{\prime}\right)\right],
\end{aligned}
$$

where $X$ stands for $R, T$, and $T_{\mathrm{d}}$, and noting that

$$
T_{m}\left(\mu, \mu^{\prime} ; \bar{\tau}\right)=T_{\mathrm{d} m}\left(\mu, \mu^{\prime} ; \bar{\tau}\right)+\frac{1}{2 \mu^{\prime}} \delta\left(\mu-\mu^{\prime}\right) \mathrm{e}^{-\bar{\tau} / \mu^{\prime}}
$$

for any azimuthal mode $m$, yields the following representations of the interaction principle in the Fourier space:

$$
\begin{aligned}
I_{\mathrm{d} m}\left(0, \mu,-\mu_{0}\right) & =\left(2-\delta_{m 0}\right) \frac{1}{\pi} \mu_{0} F_{0} R_{m}\left(\mu, \mu_{0} ; \bar{\tau}\right) \\
& +2 \int_{0}^{1} R_{m}\left(\mu, \mu^{\prime} ; \bar{\tau}\right) I_{\mathrm{d} m}\left(0,-\mu^{\prime},-\mu_{0}\right) \mu^{\prime} \mathrm{d} \mu^{\prime} \\
& +2 \int_{0}^{1} T_{m}\left(\mu, \mu^{\prime} ; \bar{\tau}\right) I_{\mathrm{d} m}\left(\bar{\tau}, \mu^{\prime},-\mu_{0}\right) \mu^{\prime} \mathrm{d} \mu^{\prime}
\end{aligned}
$$

and

$$
\begin{aligned}
I_{\mathrm{d} m}\left(\bar{\tau},-\mu,-\mu_{0}\right) & =\left(2-\delta_{m 0}\right) \frac{1}{\pi} \mu_{0} F_{0} T_{\mathrm{d} m}\left(\mu, \mu_{0} ; \bar{\tau}\right) \\
& +2 \int_{0}^{1} T_{m}\left(\mu, \mu^{\prime} ; \bar{\tau}\right) I_{\mathrm{d} m}\left(0,-\mu^{\prime},-\mu_{0}\right) \mu^{\prime} \mathrm{d} \mu^{\prime} \\
& +2 \int_{0}^{1} R_{m}\left(\mu, \mu^{\prime} ; \bar{\tau}\right) I_{\mathrm{d} m}\left(\bar{\tau}, \mu^{\prime},-\mu_{0}\right) \mu^{\prime} \mathrm{d} \mu^{\prime}
\end{aligned}
$$

The plane albedo $r$, total transmission $t$, and the spherical albedo $r_{s}$ of the layer are given by

$$
\begin{aligned}
r(\mu, \bar{\tau}) & =2 \int_{0}^{1} R_{0}\left(\mu, \mu^{\prime} ; \bar{\tau}\right) \mu^{\prime} \mathrm{d} \mu^{\prime}=2 \int_{0}^{1} R_{0}\left(\mu^{\prime}, \mu ; \bar{\tau}\right) \mu^{\prime} \mathrm{d} \mu^{\prime}, \\
t(\mu, \bar{\tau}) & =2 \int_{0}^{1} T_{0}\left(\mu, \mu^{\prime} ; \bar{\tau}\right) \mu^{\prime} \mathrm{d} \mu^{\prime}=2 \int_{0}^{1} T_{0}\left(\mu^{\prime}, \mu ; \bar{\tau}\right) \mu^{\prime} \mathrm{d} \mu^{\prime}, \\
r_{s}(\bar{\tau}) & =4 \int_{0}^{1} \int_{0}^{1} R_{0}\left(\mu, \mu^{\prime} ; \bar{\tau}\right) \mu^{\prime} \mu \mathrm{d} \mu^{\prime} \mathrm{d} \mu,
\end{aligned}
$$

while for a homogeneous layer with an underlying Lambertian surface of albedo $A$, the interaction principle is

$$
\begin{aligned}
I_{\mathrm{d} m}\left(0, \mu,-\mu_{0}\right) & =\left(2-\delta_{m 0}\right) \frac{1}{\pi} \mu_{0} F_{0} R_{A m}\left(\mu, \mu_{0} ; \bar{\tau}\right) \\
& +2 \int_{0}^{1} R_{A m}\left(\mu, \mu^{\prime} ; \bar{\tau}\right) I_{\mathrm{d} m}\left(0,-\mu^{\prime},-\mu_{0}\right) \mu^{\prime} \mathrm{d} \mu^{\prime}
\end{aligned}
$$


and

$$
\begin{aligned}
I_{\mathrm{d} m}\left(\bar{\tau},-\mu,-\mu_{0}\right) & =\left(2-\delta_{m 0}\right) \frac{1}{\pi} \mu_{0} F_{0} T_{A \mathrm{~d} m}\left(\mu, \mu_{0} ; \bar{\tau}\right) \\
& +2 \int_{0}^{1} T_{A m}\left(\mu, \mu^{\prime} ; \bar{\tau}\right) I_{\mathrm{d} m}\left(0,-\mu^{\prime},-\mu_{0}\right) \mu^{\prime} \mathrm{d} \mu^{\prime},
\end{aligned}
$$

where

$$
\begin{aligned}
R_{A m}\left(\mu, \mu^{\prime} ; \bar{\tau}\right) & =R_{m}\left(\mu, \mu^{\prime} ; \bar{\tau}\right)+\delta_{m 0} \frac{A}{1-A r_{s}} t(\mu, \bar{\tau}) t\left(\mu^{\prime}, \bar{\tau}\right), \\
T_{A m}\left(\mu, \mu^{\prime} ; \bar{\tau}\right) & =T_{m}\left(\mu, \mu^{\prime} ; \bar{\tau}\right)+\delta_{m 0} \frac{A}{1-A r_{s}} r(\mu, \bar{\tau}) t\left(\mu^{\prime}, \bar{\tau}\right), \\
T_{A \mathrm{~d} m}\left(\mu, \mu_{0} ; \bar{\tau}\right) & =T_{\mathrm{d} m}\left(\mu, \mu_{0} ; \bar{\tau}\right)+\delta_{m 0} \frac{A}{1-A r_{s}} r(\mu, \bar{\tau}) t\left(\mu_{0}, \bar{\tau}\right) .
\end{aligned}
$$

For a pertinent and mathematical elegant description of the radiative transfer in a continuous setting including the definitions of the reflection and transmission functions, as well as the derivation of the interaction principle equation, we refer to [32].

In a discrete setting, the interaction principle equation relates the outcoming radiances $\widehat{\mathbf{i}}_{\mathrm{d}}^{+}(0)$ and $\widehat{\mathbf{i}}_{\mathrm{d}}^{-}(\bar{\tau})$ to the incoming radiances $\widehat{\mathbf{i}}_{\mathrm{d}}^{-}(0)$ and $\widehat{\mathbf{i}}_{\mathrm{d}}^{+}(\bar{\tau})$ through the reflection and transmission matrices $\widehat{\mathbf{R}}$ and $\widehat{\mathbf{T}}$, respectively. Transforming the solution representations of the matrix exponential method into a form which resembles the interaction principle equation, equivalent expressions for the reflection and transmission matrices can be obtained. This derivation can be regarded as a conversion of the initial value problem of the matrix exponential method into a two-point boundary value problem (the incoming radiances $\widehat{\mathbf{i}}_{\mathrm{d}}^{-}(0)$ and $\widehat{\mathbf{i}}_{\mathrm{d}}^{+}(\bar{\tau})$ are specified). In the framework of the matrix exponential formalism, the reflection and transmission matrices are introduced in a natural way, and as they are well behaved and bounded, the numerical instability is avoided. In the matrix operator method and for a multi-layered atmosphere, the reflection matrix of the entire atmosphere is computed recursively from the reflection and transmission matrices of each layer by using the adding algorithm.

\subsection{Reflection and transmission matrices of a homogeneous layer}

First representations of the reflection and transmission matrices can be obtained from layer equation (56). Expressing (56) in terms of the upward and downward radiance vectors $\widehat{\mathbf{i}}_{\mathrm{d}}^{ \pm}(0)$ and $\widehat{\mathbf{i}}_{\mathrm{d}}^{ \pm}(\bar{\tau})$, we obtain the interaction principle equation

$$
\left[\begin{array}{c}
\widehat{\mathbf{i}}_{\mathrm{d}}^{+}(0) \\
\widehat{\mathbf{i}}_{\mathrm{d}}^{-}(\bar{\tau})
\end{array}\right]=\left[\begin{array}{cc}
\widehat{\mathbf{R}} & \widehat{\mathbf{T}} \\
\widehat{\mathbf{T}} & \widehat{\mathbf{R}}
\end{array}\right]\left[\begin{array}{c}
\widehat{\mathbf{i}}_{\mathrm{d}}^{-}(0) \\
\widehat{\mathbf{i}}_{\mathrm{d}}^{+}(\bar{\tau})
\end{array}\right]+\left[\begin{array}{c}
\widehat{\boldsymbol{\Sigma}}_{+} \\
\widehat{\boldsymbol{\Sigma}}_{-}
\end{array}\right],
$$

where $\widehat{\mathbf{R}}=\widehat{\mathbf{R}}(\bar{\tau}), \widehat{\mathbf{T}}=\widehat{\mathbf{T}}(\bar{\tau})$, and

$$
\begin{gathered}
{\left[\begin{array}{cc}
\widehat{\mathbf{R}} & \widehat{\mathbf{T}} \\
\widehat{\mathbf{T}} & \widehat{\mathbf{R}}
\end{array}\right]=\left[\begin{array}{cc}
-\boldsymbol{\Gamma}(\bar{\tau}) \overline{\mathbf{V}}_{+}^{T} & -\overline{\mathbf{V}}_{-}^{T} \\
\overline{\mathbf{V}}_{-}^{T} & \boldsymbol{\Gamma}(\bar{\tau}) \overline{\mathbf{V}}_{+}^{T}
\end{array}\right]^{-1}\left[\begin{array}{cc}
-\boldsymbol{\Gamma}(\bar{\tau}) \overline{\mathbf{V}}_{-}^{T} & -\overline{\mathbf{V}}_{+}^{T} \\
\overline{\mathbf{V}}_{+}^{T} & \boldsymbol{\Gamma}(\bar{\tau}) \overline{\mathbf{V}}_{-}^{T}
\end{array}\right],} \\
{\left[\begin{array}{c}
\widehat{\boldsymbol{\Sigma}}_{+} \\
\widehat{\boldsymbol{\Sigma}}_{-}
\end{array}\right]=\left[\begin{array}{cc}
-\boldsymbol{\Gamma}(\bar{\tau}) \overline{\mathbf{V}}_{+}^{T} & -\overline{\mathbf{V}}_{-}^{T} \\
\overline{\mathbf{V}}_{-}^{T} & \boldsymbol{\Gamma}(\bar{\tau}) \overline{\mathbf{V}}_{+}^{T}
\end{array}\right]^{-1} \mathbf{D}_{\mathrm{b}} \overline{\mathbf{V}}^{T} \widehat{\mathbf{b}}}
\end{gathered}
$$

By inspection of (86) it is apparent that the computation of $\widehat{\mathbf{R}}$ and $\widehat{\mathbf{T}}$ requires an inversion and a multiplication of matrices of dimension $2 N \times 2 N$. Similar expressions for $\widehat{\mathbf{R}}$ and $\widehat{\mathbf{T}}$, which however do not use the right- and left-eigenvectors technique, can be found in $[23,24]$. The $2 N \times 2 N$ matrix in the left-hand side of (86) is called extinction matrix. The extinction matrix is expressed in terms of $\widehat{\mathbf{R}}$ and $\widehat{\mathbf{T}}$, and as the propagator, it depends only on the internal properties of the homogeneous layer [22].

The computation of the reflection and transmission matrices can be halved in order. These representations, corresponding to the interaction principle equation

$$
\left[\begin{array}{c}
\widehat{\mathbf{i}}_{\mathrm{d}}^{+}(0) \\
\widehat{\mathbf{i}}_{\mathrm{d}}^{-}(\bar{\tau})
\end{array}\right]=\left[\begin{array}{cc}
\widehat{\mathbf{R}} & \widehat{\mathbf{T}} \\
\widehat{\mathbf{T}} & \widehat{\mathbf{R}}
\end{array}\right]\left[\begin{array}{c}
\widehat{\mathbf{i}}_{\mathrm{d}}^{-}(0)-\hat{\mathbf{i}}_{\mathrm{p}}^{-}(0) \\
\widehat{\mathbf{i}}_{\mathrm{d}}^{+}(\bar{\tau})-\hat{\mathbf{i}}_{\mathrm{p}}^{+}(\bar{\tau})
\end{array}\right]+\left[\begin{array}{c}
\hat{\mathbf{i}}_{\mathrm{p}}^{+}(0) \\
\hat{\mathbf{i}}_{\mathrm{p}}^{-}(\bar{\tau})
\end{array}\right],
$$

can be derived from (63)-(64), and (67)-(68), and do not necessarily require the use of the systems of normalized right and left eigenvectors. In the first case, we use (63) with $\tau=\bar{\tau}$ and (64) to express $\widehat{\mathbf{i}}_{\mathrm{d}}(\bar{\tau})=\left[\widehat{\mathbf{i}}_{\mathrm{d}}^{+}(\bar{\tau}), \widehat{\mathbf{i}}_{\mathrm{d}}^{-}(\bar{\tau})\right]^{T}$ and $\widehat{\mathbf{i}}_{\mathrm{d}}(0)=\left[\widehat{\mathbf{i}}_{\mathrm{d}}^{+}(0), \widehat{\mathbf{i}}_{\mathrm{d}}^{-}(0)\right]^{T}$, respectively, in terms of $\boldsymbol{\alpha}$ and $\boldsymbol{\beta}$; from these representations, we get

$\left[\begin{array}{c}\widehat{\mathbf{i}}_{\mathrm{d}}^{-}(0) \\ \widehat{\mathbf{i}}_{\mathrm{d}}^{+}(\bar{\tau})\end{array}\right]=\left[\begin{array}{cc}\widehat{\mathbf{V}}_{-} & \widehat{\mathbf{V}}_{+} \boldsymbol{\Gamma}(\bar{\tau}) \\ \widehat{\mathbf{V}}_{+} \boldsymbol{\Gamma}(\bar{\tau}) & \widehat{\mathbf{V}}_{-}\end{array}\right]\left[\begin{array}{c}\alpha \\ \beta\end{array}\right]+\left[\begin{array}{c}\hat{\mathbf{i}}_{\mathrm{p}}^{-}(0) \\ \hat{\mathbf{i}}_{\mathrm{p}}^{+}(\bar{\tau})\end{array}\right]$

and

$$
\left[\begin{array}{c}
\widehat{\mathbf{i}}_{\mathrm{d}}^{+}(0) \\
\hat{\mathbf{i}}_{\mathrm{d}}^{-}(\bar{\tau})
\end{array}\right]=\left[\begin{array}{cc}
\widehat{\mathbf{V}}_{+} & \widehat{\mathbf{V}}_{-} \boldsymbol{\Gamma}(\bar{\tau}) \\
\widehat{\mathbf{V}}_{-} \boldsymbol{\Gamma}(\bar{\tau}) & \widehat{\mathbf{V}}_{+}
\end{array}\right]\left[\begin{array}{c}
\alpha \\
\beta
\end{array}\right]+\left[\begin{array}{c}
\hat{\mathbf{i}}_{\mathrm{p}}^{+}(0) \\
\hat{\mathbf{i}}_{\mathrm{p}}^{-}(\bar{\tau})
\end{array}\right],
$$

and further,

$$
\begin{aligned}
{\left[\begin{array}{c}
\widehat{\mathbf{i}}_{\mathrm{d}}^{+}(0) \\
\hat{\mathbf{i}}_{\mathrm{d}}^{-}(\bar{\tau})
\end{array}\right] } & =\left[\begin{array}{cc}
\widehat{\mathbf{V}}_{+} & \widehat{\mathbf{V}}_{-} \boldsymbol{\Gamma}(\bar{\tau}) \\
\widehat{\mathbf{V}}_{-} \boldsymbol{\Gamma}(\bar{\tau}) & \widehat{\mathbf{V}}_{+}
\end{array}\right]\left[\begin{array}{cc}
\widehat{\mathbf{V}}_{-} & \widehat{\mathbf{V}}_{+} \boldsymbol{\Gamma}(\bar{\tau}) \\
\widehat{\mathbf{V}}_{+} \boldsymbol{\Gamma}(\bar{\tau}) & \widehat{\mathbf{V}}_{-}
\end{array}\right]^{-1} \\
& \times\left[\begin{array}{c}
\widehat{\mathbf{i}}_{\mathrm{d}}^{-}(0)-\hat{\mathbf{i}}_{\mathrm{p}}^{-}(0) \\
\hat{\mathbf{i}}_{\mathrm{d}}^{+}(\bar{\tau})-\hat{\mathbf{i}}_{\mathrm{p}}^{+}(\bar{\tau})
\end{array}\right]+\left[\begin{array}{c}
\hat{\mathbf{i}}_{\mathrm{p}}^{+}(0) \\
\hat{\mathbf{i}}_{\mathrm{p}}^{-}(\bar{\tau})
\end{array}\right] .
\end{aligned}
$$

Employing now the matrix identity

$$
\left[\begin{array}{ll}
\mathbf{A} & \mathbf{B} \\
\mathbf{B} & \mathbf{A}
\end{array}\right]^{-1}=\left[\begin{array}{cc}
\mathbf{C} & -\mathbf{A}^{-1} \mathbf{B C} \\
-\mathbf{A}^{-1} \mathbf{B C} & \mathbf{C}
\end{array}\right]
$$

with

$$
\mathbf{C}=\left(\mathbf{A}-\mathbf{B A}^{-1} \mathbf{B}\right)^{-1},
$$

we end up with

$$
\begin{aligned}
& \widehat{\mathbf{R}}=\left(\widehat{\mathbf{V}}_{+}-\widehat{\mathbf{V}}_{-} \boldsymbol{\Gamma} \widehat{\mathbf{V}}_{-}^{-1} \widehat{\mathbf{V}}_{+} \boldsymbol{\Gamma}\right)\left(\widehat{\mathbf{V}}_{-}-\widehat{\mathbf{V}}_{+} \boldsymbol{\Gamma} \widehat{\mathbf{V}}_{-}^{-1} \widehat{\mathbf{V}}_{+} \boldsymbol{\Gamma}\right)^{-1}, \\
& \widehat{\mathbf{T}}=\left(\widehat{\mathbf{V}}_{-} \boldsymbol{\Gamma}-\widehat{\mathbf{V}}_{+} \widehat{\mathbf{V}}_{-}^{-1} \widehat{\mathbf{V}}_{+} \boldsymbol{\Gamma}\right)\left(\widehat{\mathbf{V}}_{-}-\widehat{\mathbf{V}}_{+} \boldsymbol{\Gamma} \widehat{\mathbf{V}}_{-}^{-1} \widehat{\mathbf{V}}_{+} \boldsymbol{\Gamma}\right)^{-1},
\end{aligned}
$$


where $\boldsymbol{\Gamma}=\boldsymbol{\Gamma}(\bar{\tau})$. In the second case, we proceed analogously and use the matrix identity

$$
\left[\begin{array}{cc}
\mathbf{A} & -\mathbf{B} \\
\mathbf{A} & \mathbf{B}
\end{array}\right]^{-1}=\frac{1}{2}\left[\begin{array}{cc}
\mathbf{A}^{-1} & \mathbf{A}^{-1} \\
-\mathbf{B}^{-1} & \mathbf{B}^{-1}
\end{array}\right]
$$

to conclude that

$$
\widehat{\mathbf{R}}=\frac{1}{2}\left[\left(\widehat{\mathbf{V}}_{+}+\widehat{\mathbf{V}}_{-} \boldsymbol{\Gamma}\right)\left(\widehat{\mathbf{V}}_{-}+\widehat{\mathbf{V}}_{+} \boldsymbol{\Gamma}\right)^{-1}+\left(\widehat{\mathbf{V}}_{+}-\widehat{\mathbf{V}}_{-} \boldsymbol{\Gamma}\right)\left(\widehat{\mathbf{V}}_{-}-\widehat{\mathbf{V}}_{+} \boldsymbol{\Gamma}\right)^{-1}\right],
$$

$\widehat{\mathbf{T}}=\frac{1}{2}\left[\left(\widehat{\mathbf{V}}_{+}+\widehat{\mathbf{V}}_{-} \boldsymbol{\Gamma}\right)\left(\widehat{\mathbf{V}}_{-}+\widehat{\mathbf{V}}_{+} \boldsymbol{\Gamma}\right)^{-1}-\left(\widehat{\mathbf{V}}_{+}-\widehat{\mathbf{V}}_{-} \boldsymbol{\Gamma}\right)\left(\widehat{\mathbf{V}}_{-}-\widehat{\mathbf{V}}_{+} \boldsymbol{\Gamma}\right)^{-1}\right]$

By making use on fundamental matrix identities it can be shown that (94)-(95) and (97)-(98) are identical. On the other hand, it is apparent that (94)-(95) require 2 matrix inversions and 5 matrix multiplications, while (97)-(98) require 2 matrix inversions and 2 matrix multiplications. In this regard, the quantities which enter in the interaction principle equation (85) may be computed as follows:

1. calculate the matrices $\widehat{\mathbf{R}}$ and $\widehat{\mathbf{T}}$ from (97) and (98), respectively,

2. calculate the particular solution by means of (23) and (70), and the particular solution source vector according to

$$
\left[\begin{array}{c}
\widehat{\boldsymbol{\Sigma}}_{+} \\
\widehat{\boldsymbol{\Sigma}}_{-}
\end{array}\right]=\left[\begin{array}{c}
\widehat{\mathbf{i}}_{\mathrm{p}}^{+}(0) \\
\widehat{\mathbf{i}}_{\mathrm{p}}^{-}(\bar{\tau})
\end{array}\right]-\left[\begin{array}{cc}
\widehat{\mathbf{R}} & \widehat{\mathbf{T}} \\
\widehat{\mathbf{T}} & \widehat{\mathbf{R}}
\end{array}\right]\left[\begin{array}{c}
\widehat{\mathbf{i}}_{\mathrm{p}}^{-}(0) \\
\widehat{\mathbf{i}}_{\mathrm{p}}^{+}(\bar{\tau})
\end{array}\right] .
$$

Depending on the choice of the method for computing the spectral decomposition of the layer matrix $\widehat{\mathbf{A}}$, specific representations of $\widehat{\mathbf{R}}$ and $\widehat{\mathbf{T}}$ can be derived. In the square-root method, the matrices $\widehat{\mathbf{Q}}_{+}$and $\widehat{\mathbf{Q}}_{-}$, defined by $\widehat{\mathbf{V}}_{+}=\left(\widehat{\mathbf{Q}}_{+}+\widehat{\mathbf{Q}}_{-}\right) / 2$ and $\widehat{\mathbf{V}}_{-}=\left(\widehat{\mathbf{Q}}_{+}-\widehat{\mathbf{Q}}_{-}\right) / 2$, are related through the relation (cf. (344) of Appendix 1) $\widehat{\mathbf{Q}}_{-}=-\widehat{\mathbf{Q}}_{+}^{-T} \boldsymbol{\Lambda}$, and it can bee shown that $\widehat{\mathbf{R}}$ and $\widehat{\mathbf{T}}$ can be expressed in terms of $\widehat{\mathbf{Q}}_{+}^{-T}$ only. On the other hand, in the Cholesky method, the identity (cf. (351) of Appendix 1) $\widehat{\mathbf{Q}}_{+}^{-T}=-\widehat{\mathbf{Q}}_{-} \boldsymbol{\Lambda}$ can be used to express $\widehat{\mathbf{R}}$ and $\widehat{\mathbf{T}}$ in terms of $\widehat{\mathbf{Q}}_{-}$only. These representations which play an important role in the asymptotic theory will be derived in Section 5.4.

We conclude this section by presenting Waterman's derivation of the reflection and transmission matrices. Considering for simplicity, the interaction principle equation for the homogeneous solution (cf. (88))

$$
\left[\begin{array}{c}
\widehat{\mathbf{i}}_{\mathrm{h}}^{+}(0) \\
\widehat{\mathbf{i}}_{\mathrm{h}}^{-}(\bar{\tau})
\end{array}\right]=\left[\begin{array}{cc}
\widehat{\mathbf{R}} & \widehat{\mathbf{T}} \\
\widehat{\mathbf{T}} & \widehat{\mathbf{R}}
\end{array}\right]\left[\begin{array}{c}
\widehat{\mathbf{i}}_{\mathrm{h}}^{-}(0) \\
\widehat{\mathbf{i}}_{\mathrm{h}}^{+}(\bar{\tau})
\end{array}\right],
$$

we obtain

$$
\left[\begin{array}{c}
\widehat{\mathbf{i}}_{\mathrm{h}}^{+}(\bar{\tau}) \\
\widehat{\mathbf{i}}_{\mathrm{h}}^{-}(\bar{\tau})
\end{array}\right]=\left[\begin{array}{cc}
\widehat{\mathbf{T}}^{-1} & -\widehat{\mathbf{T}}^{-1} \widehat{\mathbf{R}} \\
\widehat{\mathbf{R}}^{-1} & \widehat{\mathbf{T}}-\widehat{\mathbf{R}}^{-1} \widehat{\mathbf{R}}
\end{array}\right]\left[\begin{array}{c}
\widehat{\mathbf{i}}_{\mathrm{h}}^{+}(0) \\
\widehat{\mathbf{i}}_{\mathrm{h}}^{-}(0)
\end{array}\right] .
$$

On the other hand, from (cf. (26))

$$
\left[\begin{array}{c}
\widehat{\mathbf{i}}_{\mathrm{h}}^{+}(\bar{\tau}) \\
\widehat{\mathbf{i}}_{\mathrm{h}}^{-}(\bar{\tau})
\end{array}\right]=\mathrm{e}^{-\hat{\mathbf{A}} \bar{\tau}}\left[\begin{array}{c}
\widehat{\mathbf{i}}_{\mathrm{h}}^{+}(0) \\
\widehat{\mathbf{i}}_{\mathrm{h}}^{-}(0)
\end{array}\right],
$$

it is apparent that the matrix exponential is identified as

$$
\mathrm{e}^{-\widehat{\mathbf{A}} \bar{\tau}}=\left[\begin{array}{cc}
\widehat{\mathbf{T}}^{-1} & -\widehat{\mathbf{T}}^{-1} \widehat{\mathbf{R}} \\
\widehat{\mathbf{R}}^{-1} & \widehat{\mathbf{T}}-\widehat{\mathbf{R}}^{-1} \widehat{\mathbf{R}}
\end{array}\right] .
$$

Flatau and Stephens [22] called (102) the fundamental relationship connecting the propagator (matrix exponential) and the extinction matrix. So basically, what we have to do is to evaluate $\exp (-\widehat{\mathbf{A}} \bar{\tau})$, to invert its upper-left-hand block to obtain $\widehat{\mathbf{T}}$, and finally, to postmultiply its lower-left-hand block by $\widehat{\mathbf{T}}$ in order to get $\widehat{\mathbf{R}}$. By means of (41) in conjunction with (28) and (37), we find

$$
\begin{gathered}
\widehat{\mathbf{T}}^{-1}=-\widehat{\mathbf{V}}_{+} \boldsymbol{\Gamma}(\bar{\tau}) \overline{\mathbf{V}}_{+}^{T}+\widehat{\mathbf{V}}_{-} \boldsymbol{\Gamma}(-\bar{\tau}) \overline{\mathbf{V}}_{-}^{T}, \\
\widehat{\mathbf{R}} \widehat{\mathbf{T}}^{-1}=-\widehat{\mathbf{V}}_{-} \boldsymbol{\Gamma}(\bar{\tau}) \overline{\mathbf{V}}_{+}^{T}+\widehat{\mathbf{V}}_{+} \boldsymbol{\Gamma}(-\bar{\tau}) \overline{\mathbf{V}}_{-}^{T}
\end{gathered}
$$

Equations (103)-(104) have been used by Waterman as a starting point for deriving the expressions of the reflection and transmission matrices for small and large values of the optical thickness. Here, we use a different approach in order to show the equivalences with (94)-(95), and so, with (97)-(98). Let us define the quantity

$\widehat{\mathbf{T}}_{0}=\overline{\mathbf{V}}_{-}^{-T} \boldsymbol{\Gamma}(\bar{\tau}) \widehat{\mathbf{V}}_{-}^{-1}$,

which is computationally stable, and let us construct the matrix product

$\widehat{\mathbf{T}}^{-1} \widehat{\mathbf{T}}_{0}=\mathbf{I}-\widehat{\mathbf{V}}_{+} \boldsymbol{\Gamma}(\bar{\tau}) \overline{\mathbf{V}}_{+}^{T} \overline{\mathbf{V}}_{-}^{-T} \boldsymbol{\Gamma}(\bar{\tau}) \widehat{\mathbf{V}}_{-}^{-1}$.

Then, we obtain

$\widehat{\mathbf{T}}_{0}^{-1} \widehat{\mathbf{T}}=\left[\mathbf{I}-\widehat{\mathbf{V}}_{+} \boldsymbol{\Gamma}(\bar{\tau}) \overline{\mathbf{V}}_{+}^{T} \overline{\mathbf{V}}_{-}^{-T} \boldsymbol{\Gamma}(\bar{\tau}) \widehat{\mathbf{V}}_{-}^{-1}\right]^{-1}$

and so,

$\widehat{\mathbf{T}}=\overline{\mathbf{V}}_{-}^{-T} \boldsymbol{\Gamma}\left(\widehat{\mathbf{V}}_{-}-\widehat{\mathbf{V}}_{+} \boldsymbol{\Gamma} \overline{\mathbf{V}}_{+}^{T} \overline{\mathbf{V}}_{-}^{-T} \boldsymbol{\Gamma}\right)^{-1}$,

$\widehat{\mathbf{R}}=\left(\widehat{\mathbf{V}}_{+}-\widehat{\mathbf{V}}_{-} \boldsymbol{\Gamma} \overline{\mathbf{V}}_{+}^{T} \overline{\mathbf{V}}_{-}^{-T} \boldsymbol{\Gamma}\right)\left(\widehat{\mathbf{V}}_{-}-\widehat{\mathbf{V}}_{+} \boldsymbol{\Gamma} \overline{\mathbf{V}}_{+}^{T} \overline{\mathbf{V}}_{-}^{-T} \boldsymbol{\Gamma}\right)^{-1}$,

where, as before, $\boldsymbol{\Gamma}=\boldsymbol{\Gamma}(\bar{\tau})$. Equations (108)-(109) seems to be new. However, employing (48) and (50) in (108)-(109), yields (94)-(95), and the mathematical equivalence between the various representations of the reflection and transmission matrices is proved.

The analytical formulas derived so far are valid for nonconservative scattering $(\omega \neq 1)$. The case of conservative scattering, which is merely of theoretical interest, is treated in Appendix 3 .

\subsection{Discrete approximations of the reflection and transmis- sion functions of a homogeneous layer}

There are several applications, e.g., asymptotic theory, in which discrete approximations of the reflection and transmission functions of a homogeneous layer are of particular interest. Considering the interaction principle (88) and passing from the scaled diffuse radiance vector $\widehat{\mathbf{i}}_{d}$ to the diffuse 
radiance vector $\mathbf{i}_{\mathrm{d}}$ according to the transformation rule (cf. (14)) $\widehat{\mathbf{i}}_{\mathrm{d}}^{ \pm}=\mathbf{W}^{\frac{1}{2}} \mathbf{M}^{-\frac{1}{2}} \mathbf{i}_{\mathrm{d}}^{ \pm}$, we obtain

$\left[\begin{array}{c}\mathbf{i}_{\mathrm{d}}^{+}(0) \\ \mathbf{i}_{\mathrm{d}}^{-}(\bar{\tau})\end{array}\right]=\left[\begin{array}{cc}\mathbf{R} & \mathbf{T} \\ \mathbf{T} & \mathbf{R}\end{array}\right]\left[\begin{array}{c}\mathbf{i}_{\mathrm{d}}^{-}(0)-\mathbf{c}_{2} \\ \mathbf{i}_{\mathrm{d}}^{+}(\bar{\tau})-\mathrm{e}^{-\bar{\tau} / \mu_{0}} \mathbf{c}_{1}\end{array}\right]+\left[\begin{array}{c}\mathbf{c}_{1} \\ \mathrm{e}^{-\bar{\tau} / \mu_{0}} \mathbf{c}_{2}\end{array}\right]$

where

$$
\begin{aligned}
& \mathbf{R}=\mathbf{M}^{\frac{1}{2}} \mathbf{W}^{-\frac{1}{2}} \widehat{\mathbf{R}} \mathbf{W}^{\frac{1}{2}} \mathbf{M}^{-\frac{1}{2}}, \\
& \mathbf{T}=\mathbf{M}^{\frac{1}{2}} \mathbf{W}^{-\frac{1}{2}} \widehat{\mathbf{T}} \mathbf{W}^{\frac{1}{2}} \mathbf{M}^{-\frac{1}{2}},
\end{aligned}
$$

and $\mathbf{c}=\left[\mathbf{c}_{1}, \mathbf{c}_{2}\right]^{T}=\mu_{0}\left(\mathbf{I}-\mu_{0} \mathbf{A}\right)^{-1} \mathbf{b}$, or blockwise, $\mathbf{c}_{1,2}=$ $\mathbf{W}^{-\frac{1}{2}} \mathbf{M}^{\frac{1}{2}} \widehat{\mathbf{c}}_{1,2}$. Further, from (75), (76) and (110) we find the relations

$$
\begin{aligned}
{\left[R_{m}\left(\mu_{k}, \mu_{l}\right)\right] } & =\mathbb{R}, \\
{\left[T_{m}\left(\mu_{k}, \mu_{l}\right)\right] } & =\mathbb{T}, \\
{\left[R_{m}\left(\mu_{k}, \mu_{0}\right)\right] } & =\mathrm{c}_{1}-\mathbf{R c}_{2}-\mathrm{e}^{-\bar{\tau} / \mu_{0}} \mathbf{T} \mathbf{c}_{1}, \\
{\left[T_{\mathrm{d} m}\left(\mu_{k}, \mu_{0}\right)\right] } & =\mathrm{e}^{-\bar{\tau} / \mu_{0}} \mathrm{c}_{2}-\mathbf{T c}_{2}-\mathrm{e}^{-\bar{\tau} / \mu_{0}} \mathbf{R c}_{1},
\end{aligned}
$$

where the matrices $\mathbb{R}$ and $\mathbb{T}$, given by

$\mathbb{R}=\frac{1}{2} \mathbf{R} \mathbf{M} \mathbf{W}^{-1}=\frac{1}{2} \mathbf{M}^{\frac{1}{2}} \mathbf{W}^{-\frac{1}{2}} \widehat{\mathbf{R}} \mathbf{W}^{-\frac{1}{2}} \mathbf{M}^{\frac{1}{2}}$

$\mathbb{T}=\frac{1}{2} \mathbf{T} \mathbf{M} \mathbf{W}^{-1}=\frac{1}{2} \mathbf{M}^{\frac{1}{2}} \mathbf{W}^{-\frac{1}{2}} \widehat{\mathbf{T}} \mathbf{W}^{-\frac{1}{2}} \mathbf{M}^{\frac{1}{2}}$,

are the discrete approximations of the reflection and transmission functions, respectively, and the vector $\mathrm{c}=\left[\mathrm{c}_{1}, \mathrm{c}_{2}\right]^{T}$ is defined through the relation $\mathbf{c}=\left(2-\delta_{m 0}\right)\left(F_{0} / \pi\right) \mu_{0}$ c. In addition, the matrix

$\mathbb{T}_{\mathrm{d}}=\mathbb{T}-\mathbf{D}$,

with

$[\mathbf{D}]_{k l}=\frac{1}{2 w_{k} \mu_{k}} \mathrm{e}^{-\bar{\tau} / \mu_{k}} \delta_{k l}$,

is the discrete approximation of the diffuse transmission function, i.e.,

$\left[T_{\mathrm{d} m}\left(\mu_{k}, \mu_{l}\right)\right]=\mathbb{T}_{\mathrm{d}}$.

Equations (117) and (118) show how to convert the scaled reflection and transmission matrices into physical functions. As the interaction principle has been formulated for the diffuse radiance, relations (115) and (116) corresponding to the incident direction can be used for checking the reflection and transmission matrix calculations. The computational process of some reflective and transmissive characteristics of the layer involves the following steps:

1. For the azimuthal mode $m=0$, compute the plane albedo vector $\mathbf{r}$ and the transmission vector $\mathbf{t}$ of the layer

$$
\begin{aligned}
& \mathbf{r}=2 \mathbb{R} \mathbf{W} \mathbf{M}^{-1} \mathbf{1}=\mathbf{R} \mathbf{1}, \\
& \mathbf{t}=2 \mathbb{W} \mathbf{W M}^{-1} \mathbf{1}=\mathbf{T} \mathbf{1},
\end{aligned}
$$

together with the spherical albedo

$$
r_{s}=4 \mathbf{v}^{T} \mathbb{R} \mathbf{v}=2 \mathbf{v}^{T} \mathbf{R} \mathbf{1},
$$

where $\mathbf{v}=\mathbf{W M}^{-1} \mathbf{1}$ and $\mathbf{1}=[1, \ldots, 1]^{T}$.

2. Compute the reflection and transmission matrices of the layer with an underlying Lambertian surface

$$
\begin{aligned}
& \mathbb{R}_{A}=\mathbb{R}+\frac{A}{1-A r_{s}} \delta_{m 0} \mathbf{t t}^{T}, \\
& \mathbb{T}_{A}=\mathbb{T}+\frac{A}{1-A r_{s}} \delta_{m 0} \mathbf{r t}^{T} .
\end{aligned}
$$

3. For the azimuthal mode $m=0$, compute the plane albedo vector of the homogeneous layer with an underlying Lambertian surface

$$
\mathbf{r}_{A}=2 \mathbb{R}_{A} \mathbf{W} \mathbf{M}^{-1} \mathbf{1}
$$

and the spherical albedo

$$
r_{S A}=4 \mathbf{v}^{T} \mathbb{R}_{A} \mathbf{v}
$$

According to (74), the total transmission function is a generalized function, or a distribution. In a discrete setting this means that in contrast to the diffuse transmission matrix $\left[T_{\mathrm{d} m}\left(\mu_{k}, \mu_{l} ; \bar{\tau}\right)\right]$, the total transmission matrix $\left[T_{m}\left(\mu_{k}, \mu_{l} ; \bar{\tau}\right)\right]$, regarded as a function of $\mu_{l}$, has a jump at $\mu_{l}=\mu_{k}$.

The discrete approximations of the reflection and transmission functions $R\left(\mu, \mu^{\prime}, \varphi-\varphi^{\prime} ; \bar{\tau}\right), T\left(\mu, \mu^{\prime}, \varphi-\varphi^{\prime} ; \bar{\tau}\right)$ and $T_{\mathrm{d}}\left(\mu, \mu^{\prime}, \varphi-\varphi^{\prime} ; \bar{\tau}\right)$ are obtained by summing up the Fourier series in the azimuth, i.e.,

$\mathbb{X}=\sum_{m=0}^{M_{\max }}\left(2-\delta_{m 0}\right) \mathbb{X}_{m} \cos \left[m\left(\varphi-\varphi^{\prime}\right)\right]$,

where $\mathbb{X}$ stands for $\mathbb{R}, \mathbb{T}$, and $\mathbb{T}_{\mathrm{d}}$. The number of azimuthal modes $M_{\max }$ is whether $M_{\max }=2 N-1$, or $M_{\max }<2 N-1$. In the second case, an azimuthal convergence test over the diagonal elements of $\mathbb{R}$ and $\mathbb{T}_{d}$ is performed. A stronger test may involve the convergence of the Frobenius norms of $\mathbb{R}$ and $\mathbb{T}_{d}$.

For highly peaked phase functions the delta-M method [33] can be used. In this case, the matrices $\mathbf{S}_{ \pm}$, and hence, the matrices $\widehat{\mathbf{V}}_{ \pm}$in (97)-(98) are altered by modifying the Legendre expansion coefficients $\chi_{n}$ of the phase function $p\left(\mu, \mu^{\prime}, \varphi-\varphi^{\prime}\right)$ and the single scattering albedo $\omega$ according to

$\chi_{n}^{\star}=\frac{1}{1-f}\left(\chi_{n}-2 f\right)$

and

$\omega^{\star}=\frac{1-f}{1-f \omega} \omega$,

respectively, while the diagonal matrix $\boldsymbol{\Gamma}=\left[\mathrm{e}^{-\lambda_{k} \bar{\tau}}\right]$ in (97)(98) is altered by modifying the optical thickness $\bar{\tau}$ according to $\bar{\tau}^{\star}=(1-f \omega) \bar{\tau}$. Here, the truncation factor $f$ is defined by $f=(1 / 2) \chi_{2 N}$. The delta-M method enhances the convergence of $\mathbb{R}$ and $\mathbb{T}_{d}$ when compared with the corresponding results obtained in the absence of truncation. 
The delta-M method can be used in conjunction with the truncated-plus-single-scattering (TMS) method [34]. The application of the TMS correction to the reflection matrix of a semi-infinite atmosphere was discussed in [35]. For a layer of finite optical thickness, the TMS corrections of the reflection and transmission matrices use the corresponding functions in the single-scattering approximation

$$
\begin{aligned}
R_{\mathrm{SS}}\left(\mu, \mu_{0}, \varphi-\varphi_{0} ; \bar{\tau}\right) & =\frac{1}{4\left(\mu+\mu_{0}\right)} \omega p\left(\mu,-\mu_{0}, \varphi-\varphi_{0}\right) \\
& \times\left[1-\mathrm{e}^{-\left(1 / \mu_{0}+1 / \mu\right) \bar{\tau}}\right], \\
T_{\mathrm{SSd}}\left(\mu, \mu_{0}, \varphi-\varphi_{0} ; \bar{\tau}\right) & =\frac{1}{4\left(\mu-\mu_{0}\right)} \omega p\left(-\mu,-\mu_{0}, \varphi-\varphi_{0}\right) \\
& \times\left(\mathrm{e}^{-\bar{\tau} / \mu}-\mathrm{e}^{-\bar{\tau} / \mu_{0}}\right),
\end{aligned}
$$

For example, the TMS correction of the reflection function is

$$
\triangle R\left(\mu, \mu_{0}, \varphi-\varphi_{0} ; \bar{\tau}\right)=R_{\mathrm{SS}}^{\mathrm{e}}\left(\mu, \mu_{0}, \varphi-\varphi_{0} ; \bar{\tau}\right)-R_{\mathrm{SS}}^{\mathrm{t}}\left(\mu, \mu_{0}, \varphi-\varphi_{0} ; \bar{\tau}\right),
$$

where,

$$
\begin{aligned}
R_{\mathrm{SS}}^{\mathrm{e}}\left(\mu, \mu_{0}, \varphi-\varphi_{0} ; \bar{\tau}\right) & =\frac{1}{4\left(\mu+\mu_{0}\right)} \frac{\omega}{1-f \omega} p\left(\mu,-\mu_{0}, \varphi-\varphi_{0}\right) \\
& \times\left[1-\mathrm{e}^{-\left(1 / \mu_{0}+1 / \mu\right) \bar{\tau}^{\star}}\right] \\
p\left(\mu,-\mu_{0}, \varphi-\varphi_{0}\right) & =\sum_{n=0}^{N_{\max }} \sqrt{\frac{2 n+1}{2}} \chi_{n} P_{n}(\cos \Theta)
\end{aligned}
$$

and

$$
\begin{aligned}
R_{\mathrm{SS}}^{\mathrm{t}}\left(\mu, \mu_{0}, \varphi-\varphi_{0} ; \bar{\tau}\right) & =\frac{1}{4\left(\mu+\mu_{0}\right)} \omega^{\star} p^{\star}\left(\mu,-\mu_{0}, \varphi-\varphi_{0}\right) \\
& \times\left[1-\mathrm{e}^{-\left(1 / \mu_{0}+1 / \mu\right) \bar{\tau}^{\star}}\right],
\end{aligned}
$$

correspond to the "exact" and truncated phase functions, respectively. In (135), $P_{n}$ are the normalized Legendre polynomials, $\cos \Theta=-\mu \mu_{0}+\sqrt{1-\mu^{2}} \sqrt{1-\mu_{0}^{2}} \cos \left(\varphi-\varphi_{0}\right), N_{\max }>$ $2 N-1$ is the number of all expansion coefficients, and $\bar{\tau}^{\star}$ is the delta-M scaled optical thickness. The truncated phase function $p^{\star}(\Theta)$ in (136) can be computed in two different ways. If an azimuthal convergence test is performed $\left(M_{\max }<\right.$ $2 N-1), p^{\star}(\Theta)$ is computed as

$$
\begin{aligned}
p^{\star}\left(\mu,-\mu_{0}, \varphi-\varphi_{0}\right) & =\sum_{m=0}^{M_{\max }}\left(2-\delta_{m 0}\right) p_{m}^{\star}\left(\mu,-\mu_{0}\right) \cos \left[m\left(\varphi-\varphi_{0}\right)\right] \\
p_{m}^{\star}\left(\mu,-\mu_{0}\right) & =\sum_{n=m}^{2 N-1} \chi_{n}^{\star} P_{n}^{m}(\mu) P_{n}^{m}\left(-\mu_{0}\right),
\end{aligned}
$$

where $P_{n}^{m}$ are the normalized Legendre functions, and otherwise, as

$$
p^{\star}(\Theta)=\sum_{n=0}^{2 N-1} \sqrt{\frac{2 n+1}{2}} \chi_{n}^{\star} P_{n}(\cos \Theta) .
$$

To analyze the accuracy of the TMS method in computing the reflection and transmission functions we perform numerical simulations of the Earth Polychromatic Imaging Camera (EPIC) measurements in the oxygen A-band absorption channel at $764 \mathrm{~nm}$ (Channel 9). Note that this channel is used for cloud parameters retrieval, and that the EPIC instrument, onboard the Deep Space Climate Observatory (DSCOVR), measures from the L1 Lagrangian point, at which the satellite remains near the Sun-Earth line. For an atmospheric layer consisting of oxygen molecules and a water cloud, we assume that the radiative transfer in the layer involves, in addition to cloud scattering and absorption, oxygen absorption. More precisely, we neglect the molecular Rayleigh scattering, that is, for the homogenized layer, we use the approximations $\sigma_{\mathrm{ext}} \approx \sigma_{\mathrm{ext}}^{\mathrm{c}}+\sigma_{\mathrm{abs}}^{\mathrm{g}}, \sigma_{\mathrm{sct}} \approx \sigma_{\mathrm{sct}}^{\mathrm{c}}, \chi_{n} \approx \chi_{n}^{\mathrm{c}}$, where $\sigma_{\mathrm{ext}}^{\mathrm{c}}, \sigma_{\mathrm{sct}}^{\mathrm{c}}$ and $\chi_{n}^{\mathrm{c}}$ are the extinction coefficient, scattering coefficient and the phase function expansion coefficients of the cloud, while $\sigma_{\text {abs }}^{\mathrm{g}}$ is the absorption coefficient of the oxygen gas. Perhaps it should be pointed out that the high oxygen absorption (computed by line-by-line calculations), yields a large interval of variation of the single scattering albedo of the homogenized layer (between 0.2 and 0.999) As the Sun is in the back of the instrument, the observed scattering angle is close to the backscattering direction. To model the scattering in the backward region, we take $\mu=\mu_{0}$, and as in [36], we choose $\varphi-\varphi_{0}=176^{\circ}$. In Table 1 we show the relative errors in $R$ and $T_{\mathrm{d}}$, defined by

$\varepsilon_{\mathrm{R}}=\sqrt{\frac{\int_{0}^{1}\left[R\left(\mu, \mu, \varphi-\varphi_{0} ; \bar{\tau}\right)-R_{\mathrm{ref}}\left(\mu, \mu, \varphi-\varphi_{0} ; \bar{\tau}\right)\right]^{2} \mathrm{~d} \mu}{\int_{0}^{1} R_{\mathrm{ref}}^{2}\left(\mu, \mu, \varphi-\varphi_{0} ; \bar{\tau}\right) \mathrm{d} \mu}}$

and similarly for $\varepsilon_{\mathrm{T}}$, for the delta-M method and the delta-M method with TMS correction. The optical thickness is $\bar{\tau}=5$, the single scattering albedo is $\omega=0.85$, and the phase function corresponds to a water-cloud model with a Gamma size distribution

$P(a) \propto a^{\alpha} \exp \left[-\alpha\left(\frac{a}{a_{\mathrm{mod}}}\right)\right]$

of parameters $a_{\mathrm{mod}}=10 \mu \mathrm{m}$ and $\alpha=6$. The droplet size ranges between 0.02 and $50.0 \mu \mathrm{m}$, and the reference values $R_{\text {ref }}$ and $T_{\text {dref }}$ correspond to $N_{\text {rank }}=565$. The results demonstrate that the TMS method requires less discrete ordinates $N$ as the standard delta-M method. This observation implies that the TMS method improves the accuracy of reflection and transmission functions calculations.

This model based on an eigendecomposition method for computing the matrix exponential is used as a reference for testing the approximate representations of the reflection and transmission matrices in the cases of thin and thick layers. In particular, for the EPIC instrument, we compute the relative errors

$$
\varepsilon_{\mathrm{X}}=\sqrt{\frac{\sum_{k=1}^{N}\left([\mathbb{X}]_{k k}-\left[\mathbb{X}_{\mathrm{ref}}\right]_{k k}\right)^{2}}{\sum_{k=1}^{N}\left[\mathbb{X}_{\mathrm{ref}}\right]_{k k}^{2}}},
$$

where as before, $\mathbb{X}$ stands for $\mathbb{R}$ and $\mathbb{T}$, and $\varphi-\varphi_{0}=176^{\circ}$. 
Table 1: Relative errors $\varepsilon_{\mathrm{R}}$ and $\varepsilon_{\mathrm{T}}$ for the delta-M method and the delta-M method with TMS correction, and for different values of the number of discrete ordinates per hemisphere $N$.

\begin{tabular}{clllll}
\hline \multirow{2}{*}{$N$} & \multicolumn{2}{c}{ Delta-M } & & \multicolumn{2}{c}{ Delta-M and TMS } \\
\cline { 2 - 3 } \cline { 5 - 6 } & $\varepsilon_{\mathrm{R}}$ & $\varepsilon_{\mathrm{T}}$ & & $\varepsilon_{\mathrm{R}}$ & $\varepsilon_{\mathrm{T}}$ \\
\hline 128 & $3.80 \mathrm{e}-4$ & $4.44 \mathrm{e}-4$ & & $3.53 \mathrm{e}-4$ & $4.44 \mathrm{e}-4$ \\
64 & $1.64 \mathrm{e}-2$ & $2.06 \mathrm{e}-3$ & & $1.41 \mathrm{e}-3$ & $1.91 \mathrm{e}-3$ \\
56 & $1.97 \mathrm{e}-2$ & $2.81 \mathrm{e}-3$ & & $1.61 \mathrm{e}-3$ & $2.56 \mathrm{e}-3$ \\
48 & $2.28 \mathrm{e}-2$ & $4.24 \mathrm{e}-3$ & & $2.21 \mathrm{e}-3$ & $3.70 \mathrm{e}-3$ \\
32 & $4.34 \mathrm{e}-2$ & $8.63 \mathrm{e}-3$ & & $3.76 \mathrm{e}-3$ & $7.73 \mathrm{e}-3$ \\
16 & $6.12 \mathrm{e}-2$ & $2.85 \mathrm{e}-2$ & & $2.42 \mathrm{e}-2$ & $2.66 \mathrm{e}-2$ \\
8 & $1.95 \mathrm{e}-1$ & $1.05 \mathrm{e}-1$ & & $6.71 \mathrm{e}-2$ & $8.97 \mathrm{e}-2$ \\
\hline
\end{tabular}

\subsection{Reflection and transmission matrices of a homogeneous thin layer}

For optically thin layers, the Padé and Taylor series approximations to the matrix exponential can be used for computing the reflection and transmission matrices and so, to avoid the solution of an eigenvalue problem. The interest in such small values of $\bar{\tau}$ stems from the possibility of using these approximations to generate starting values for the doubling method. Parenthetically, we note that the basic recursion relations of the doubling method can be obtained by using the matrix exponential formalism. Indeed, setting $\widehat{\mathbf{R}}_{1}=\widehat{\mathbf{R}}(\bar{\tau})$ and $\widehat{\mathbf{T}}_{1}=\widehat{\mathbf{T}}(\bar{\tau})$ for the layer of optical thickness $\bar{\tau}$, and $\widehat{\mathbf{R}}_{2}=\widehat{\mathbf{R}}(2 \bar{\tau})$ and $\widehat{\mathbf{T}}_{2}=\widehat{\mathbf{T}}(2 \bar{\tau})$ for the layer of optical thickness $2 \bar{\tau}$, we express (102) for the layers $\bar{\tau}$ and $2 \bar{\tau}$, as

$\mathrm{e}^{-\hat{\mathbf{A}} \bar{\tau}}=\left[\begin{array}{cc}\widehat{\mathbf{T}}_{1}^{-1} & -\widehat{\mathbf{T}}_{1}^{-1} \widehat{\mathbf{R}}_{1} \\ \widehat{\mathbf{R}}_{1} \widehat{\mathbf{T}}_{1}^{-1} & \widehat{\mathbf{T}}_{1}-\widehat{\mathbf{R}}_{1} \widehat{\mathbf{T}}_{1}^{-1} \widehat{\mathbf{R}}_{1}\end{array}\right]$

and

$\mathrm{e}^{-2 \hat{\mathbf{A}} \bar{\tau}}=\left[\begin{array}{cc}\widehat{\mathbf{T}}_{2}^{-1} & -\widehat{\mathbf{T}}_{2}^{-1} \widehat{\mathbf{R}}_{2} \\ \widehat{\mathbf{R}}_{2} \widehat{\mathbf{T}}_{2}^{-1} & \widehat{\mathbf{T}}_{2}-\widehat{\mathbf{R}}_{2} \widehat{\mathbf{T}}_{2}^{-1} \widehat{\mathbf{R}}_{2}\end{array}\right]$,

respectively, and use the identity $\exp (-2 \hat{\mathbf{A}} \bar{\tau})=[\exp (-\hat{\mathbf{A}} \bar{\tau})]^{2}$, to obtain

$$
\left[\begin{array}{cc}
\widehat{\mathbf{T}}_{2}^{-1} & -\widehat{\mathbf{T}}_{2}^{-1} \widehat{\mathbf{R}}_{2} \\
\widehat{\mathbf{R}}_{2} \widehat{\mathbf{T}}_{2}^{-1} & \widehat{\mathbf{T}}_{2}-\widehat{\mathbf{R}}_{2} \widehat{\mathbf{T}}_{2}^{-1} \widehat{\mathbf{R}}_{2}
\end{array}\right]=\left[\begin{array}{cc}
\widehat{\mathbf{T}}_{1}^{-1} & -\widehat{\mathbf{T}}_{1}^{-1} \widehat{\mathbf{R}}_{1} \\
\widehat{\mathbf{R}}_{1} \widehat{\mathbf{T}}_{1}^{-1} & \widehat{\mathbf{T}}_{1}-\widehat{\mathbf{R}}_{1} \widehat{\mathbf{T}}_{1}^{-1} \widehat{\mathbf{R}}_{1}
\end{array}\right]^{2} .
$$

Solving for $\widehat{\mathbf{R}}_{2}$ and $\widehat{\mathbf{T}}_{2}$, we get

$$
\begin{aligned}
\widehat{\mathbf{R}}_{2} & =\widehat{\mathbf{R}}_{1}+\widehat{\mathbf{T}}_{1} \widehat{\mathbf{R}}_{1}\left(\mathbf{I}-\widehat{\mathbf{R}}_{1}^{2}\right)^{-1} \widehat{\mathbf{T}}_{1}, \\
\widehat{\mathbf{T}}_{2} & =\widehat{\mathbf{T}}_{2}\left(\mathbf{I}-\widehat{\mathbf{R}}_{1}^{2}\right)^{-1} \widehat{\mathbf{T}}_{1},
\end{aligned}
$$

which are the recursion relations of the doubling method.

\subsubsection{Padé approximation}

In radiative transfer, the Padé approximation has been suggested by Flatau and Stephens [22] for computing the extinction matrix and the source function integral of a layer. More recently, McGararagh and Gabriel [37] used this approximation in connection with the matrix operator method. Essentially, the $n$th diagonal Padé approximation to the exponential of the matrix $\widehat{\mathbf{A}} \tau$ is defined as [38]

$\mathrm{e}^{-\widehat{\mathbf{A}} \tau} \approx\left[\mathbf{D}_{n}(\widehat{\mathbf{A}} \tau)\right]^{-1} \mathbf{N}_{n}(\widehat{\mathbf{A}} \tau)$,

where $\mathbf{D}_{n}(\widehat{\mathbf{A}} \tau)$ and $\mathbf{N}_{n}(\widehat{\mathbf{A}} \tau)$ are polynomials in $\widehat{\mathbf{A}} \tau$ of degree $n$, given by

$\mathbf{D}_{n}(\widehat{\mathbf{A}} \tau)=\sum_{k=0}^{n} c_{k} \tau^{k} \widehat{\mathbf{A}}^{k}$,

$\mathbf{N}_{n}(\widehat{\mathbf{A}} \tau)=\sum_{k=0}^{n}(-1)^{k} c_{k} \tau^{k} \widehat{\mathbf{A}}^{k}$,

and

$c_{k}=\frac{(2 n-k) ! n !}{(2 n) ! k !(n-k) !}$.

From (148) and (149) it is readily seen that $\mathbf{N}_{n}(\widehat{\mathbf{A}} \tau)=\mathbf{D}_{n}(-\widehat{\mathbf{A}} \tau)$. To compute $\mathbf{D}_{n}(\widehat{\mathbf{A}} \tau)$ and $\mathbf{N}_{n}(\widehat{\mathbf{A}} \tau)$ we have to compute powers of $\widehat{\mathbf{A}}$. By taking advantage of the block symmetries within $\widehat{\mathbf{A}}$ (cf. (6)), we find

$\widehat{\mathbf{A}}^{k}=\left[\begin{array}{cc}\mathbf{X}_{k} & \mathbf{Y}_{k} \\ (-1)^{k} \mathbf{Y}_{k} & (-1)^{k} \mathbf{X}_{k}\end{array}\right]$,

where for $k \geq 2$, the matrices $\mathbf{X}_{k}$ and $\mathbf{Y}_{k}$ are computed recursively as

$\mathbf{X}_{k}=\mathbf{X}_{k-1} \widehat{\mathbf{A}}_{11}-\mathbf{Y}_{k-1} \widehat{\mathbf{A}}_{12}$,

$\mathbf{Y}_{k}=\mathbf{X}_{k-1} \widehat{\mathbf{A}}_{12}-\mathbf{Y}_{k-1} \widehat{\mathbf{A}}_{11}$,

with the initial values

$\mathbf{X}_{1}=\widehat{\mathbf{A}}_{11}$,

$\mathbf{Y}_{1}=\widehat{\mathbf{A}}_{12}$.

The coefficients $c_{k}$ are also computed recursively by means of

$c_{k}=\frac{n-k+1}{k(2 n-k+1)} c_{k-1}$,

with the initial value $c_{1}=1 / 2$. Accounting of (151), the matrices $\mathbf{D}_{n}(\widehat{\mathbf{A}} \tau)$ and $\mathbf{N}_{n}(\widehat{\mathbf{A}} \tau)$ of (148) and (149), respectively, can be written as

$\mathbf{D}_{n}(\widehat{\mathbf{A}} \tau)=\left[\begin{array}{cc}\mathbf{I}+\mathbf{X}_{n}^{+} & \mathbf{Y}_{n}^{+} \\ \mathbf{Y}_{n}^{-} & \mathbf{I}+\mathbf{X}_{n}^{-}\end{array}\right]$,
$\mathbf{N}_{n}(\widehat{\mathbf{A}} \tau)=\left[\begin{array}{cc}\mathbf{I}+\mathbf{X}_{n}^{-} & \mathbf{Y}_{n}^{-} \\ \mathbf{Y}_{n}^{+} & \mathbf{I}+\mathbf{X}_{n}^{+}\end{array}\right]$,

where

$$
\begin{aligned}
& \mathbf{X}_{n}^{ \pm}=\sum_{k=1}^{n}( \pm 1)^{k} c_{k} \tau^{k} \mathbf{X}_{k} \\
& \mathbf{Y}_{n}^{ \pm}=\sum_{k=1}^{n}( \pm 1)^{k} c_{k} \tau^{k} \mathbf{Y}_{k} .
\end{aligned}
$$


Inserting (147) together with (157) and (158) into the homogeneous solution representation (26) yields

$\widehat{\mathbf{R}}_{n}=\mathbf{H}_{n}\left(\mathbf{I}+\mathbf{X}_{n}^{+}\right)-\mathbf{G}_{n} \mathbf{Y}_{n}^{-}$,

$\widehat{\mathbf{T}}_{n}=\mathbf{G}_{n}\left(\mathbf{I}+\mathbf{X}_{n}^{+}\right)-\mathbf{H}_{n} \mathbf{Y}_{n}^{-}$,

with

$\mathbf{G}_{n}=\left(\mathbf{E}_{n}-\mathbf{F}_{n} \mathbf{E}_{n}^{-1} \mathbf{F}_{n}\right)^{-1}$,

$\mathbf{H}_{n}=\mathbf{E}_{n}^{-1} \mathbf{F}_{n} \mathbf{G}_{n}$,

and

$\mathbf{E}_{n}=\mathbf{I}+\mathbf{X}_{n}^{-}$,

$\mathbf{F}_{n}=\mathbf{Y}_{n}^{+}$.

Equations (161) and (162) give the $n$ th-order Padé approximations to the reflection and transmission matrices. In the case $n=1$, we find $\mathbf{X}_{1}^{ \pm}= \pm(\bar{\tau} / 2) \widehat{\mathbf{A}}_{11}$ and $\mathbf{Y}_{1}^{ \pm}= \pm(\bar{\tau} / 2) \widehat{\mathbf{A}}_{12}$. As a result, we obtain $\mathbf{E}_{1}=\mathbf{I}-(\bar{\tau} / 2) \widehat{\mathbf{A}}_{11}$ and $\mathbf{F}_{1}=(\bar{\tau} / 2) \widehat{\mathbf{A}}_{12}$, and so,

$\widehat{\mathbf{R}}_{1}=\mathbf{H}_{1}\left(\mathbf{I}+\frac{\bar{\tau}}{2} \widehat{\mathbf{A}}_{11}\right)+\frac{\bar{\tau}}{2} \mathbf{G}_{1} \widehat{\mathbf{A}}_{12}$,

$\widehat{\mathbf{T}}_{1}=\mathbf{G}_{1}\left(\mathbf{I}+\frac{\overline{\boldsymbol{\tau}}}{2} \widehat{\mathbf{A}}_{11}\right)+\frac{\bar{\tau}}{2} \mathbf{H}_{1} \widehat{\mathbf{A}}_{12}$.

Further, approximating $\mathbf{E}_{1}^{-1} \approx \mathbf{I}+(\bar{\tau} / 2) \widehat{\mathbf{A}}_{11}$, and retaining only the first-order terms in the Neumann series of the inverse in (163), gives $\mathbf{G}_{1}=\mathbf{I}+(\bar{\tau} / 2) \widehat{\mathbf{A}}_{11}$ and $\mathbf{H}_{1}=(\bar{\tau} / 2) \widehat{\mathbf{A}}_{12}$, and ultimately

$\widehat{\mathbf{R}}_{1}=\bar{\tau} \widehat{\mathbf{A}}_{12}$ and $\widehat{\mathbf{T}}_{1}=\mathbf{I}+\bar{\tau} \widehat{\mathbf{A}}_{11}$.

This is the infinitesimal generator initialization scheme of Grant and Hunt [39] (see (181) below).

\subsubsection{Taylor series approximation}

The Taylor series approximation uses the definition of the matrix exponential, namely

$\mathrm{e}^{-\widehat{\mathbf{A}} \tau} \approx \mathbf{I}+\sum_{k=1}^{n}(-1)^{k} \frac{1}{k !} \tau^{k} \widehat{\mathbf{A}}^{k}$

Accounting of (151), we obtain

$\mathrm{e}^{-\widehat{\mathbf{A}} \bar{\tau}} \approx \mathbf{I}+\sum_{k=1}^{n}(-1)^{k} \frac{1}{k !} \bar{\tau}^{k}\left[\begin{array}{cc}\mathbf{X}_{k} & \mathbf{Y}_{k} \\ (-1)^{k} \mathbf{Y}_{k} & (-1)^{k} \mathbf{X}_{k}\end{array}\right]$

while from (142) we get

$$
\begin{aligned}
\widehat{\mathbf{T}}^{-1} & \approx \widehat{\mathbf{T}}_{n}^{-1}=\mathbf{I}+\sum_{k=1}^{n} \bar{\tau}^{k} \mathbf{E}_{k}, \\
\widehat{\mathbf{R}} \widehat{\mathbf{T}}^{-1} & \approx \widehat{\mathbf{R}}_{n} \widehat{\mathbf{T}}_{n}^{-1}=\sum_{k=1}^{n} \bar{\tau}^{k} \mathbf{F}_{k},
\end{aligned}
$$

where the matrices $\mathbf{E}_{k}$ and $\mathbf{F}_{k}$ are now given by

$$
\mathbf{E}_{k}=\frac{(-1)^{k}}{k !} \mathbf{X}_{k} \text { and } \mathbf{F}_{k}=\frac{1}{k !} \mathbf{Y}_{k}
$$

respectively. Using (172) and (173), and seeking for expansions of the form

$\widehat{\mathbf{T}}_{n}=\mathbf{I}+\sum_{k=1}^{n} \bar{\tau}^{k} \mathbf{G}_{k}$

$\widehat{\mathbf{R}}_{n}=\sum_{k=1}^{n} \bar{\tau}^{k} \mathbf{H}_{k}$,

we find that the matrices $\mathbf{G}_{n}$ and $\mathbf{H}_{n}$ in (175) and (176) can be computed recursively as

$\mathbf{G}_{1}=-\mathbf{E}_{1}$,

$\mathbf{G}_{n}=-\mathbf{E}_{n}-\sum_{k=1}^{n-1} \mathbf{E}_{n-k} \mathbf{G}_{k}, n \geq 2$,

and

$\mathbf{H}_{1}=\mathbf{F}_{1}$,

$\mathbf{H}_{n}=\mathbf{F}_{n}+\sum_{k=1}^{n-1} \mathbf{F}_{n-k} \mathbf{G}_{k}, n \geq 2$,

respectively. Equations (175) and (176) give the $n$ th-order Taylor series approximations to the transmission and reflection matrices. In the case $n=1$, we obtain the infinitesimal generator initialization scheme of Grant and Hunt [39],

$\widehat{\mathbf{T}}_{1}=\mathbf{I}+\bar{\tau} \widehat{\mathbf{A}}_{11}, \widehat{\mathbf{R}}_{1}=\bar{\tau} \widehat{\mathbf{A}}_{12}$,

in the case $n=2$, we obtain the expanded diamond initialization scheme of Wiscombe [40],

$\widehat{\mathbf{T}}_{2}=\widehat{\mathbf{T}}_{1}+\frac{1}{2} \bar{\tau}^{2}\left(\widehat{\mathbf{A}}_{11}^{2}+\widehat{\mathbf{A}}_{12}^{2}\right)$,

$\widehat{\mathbf{R}}_{2}=\widehat{\mathbf{R}}_{1}+\frac{1}{2} \bar{\tau}^{2}\left(\widehat{\mathbf{A}}_{11} \widehat{\mathbf{A}}_{12}+\widehat{\mathbf{A}}_{12} \widehat{\mathbf{A}}_{11}\right)$,

and finally, in the case $n=3$, we obtain the scheme of Waterman [21],

$\widehat{\mathbf{T}}_{3}=\widehat{\mathbf{T}}_{2}+\frac{1}{6} \bar{\tau}^{3}\left(\widehat{\mathbf{A}}_{11}^{3}+2 \widehat{\mathbf{A}}_{12}^{2} \widehat{\mathbf{A}}_{11}+2 \widehat{\mathbf{A}}_{11} \widehat{\mathbf{A}}_{12}^{2}+\widehat{\mathbf{A}}_{12} \widehat{\mathbf{A}}_{11} \widehat{\mathbf{A}}_{12}\right)$,

$\widehat{\mathbf{R}}_{3}=\widehat{\mathbf{R}}_{2}+\frac{1}{6} \bar{\tau}^{3}\left(2 \widehat{\mathbf{A}}_{12}^{3}+\widehat{\mathbf{A}}_{11}^{2} \widehat{\mathbf{A}}_{12}+\widehat{\mathbf{A}}_{12} \widehat{\mathbf{A}}_{11}^{2}+2 \widehat{\mathbf{A}}_{11} \widehat{\mathbf{A}}_{12} \widehat{\mathbf{A}}_{11}\right)$.

The Padé and Taylor series approximations are based on the computation of powers of $\widehat{\mathbf{A}}$, for which we used (151)(155). An alternative approach for computing $\widehat{\mathbf{A}}^{k}$, which exploits more efficiently the symmetries of the matrix $\widehat{\mathbf{A}}$, has been proposed by Waterman [21], and can also be found in Flatau and Stephens [22], and McGarragh and Gabriel [37]. The idea is to consider the similarity transformation $\widetilde{\mathbf{A}}=$ $\mathbf{L}^{-1} \widehat{\mathbf{A}} \mathbf{L}$, with

$\mathbf{L}=\sqrt{\frac{1}{2}}\left[\begin{array}{cc}\mathbf{I} & \mathbf{I} \\ \mathbf{I} & -\mathbf{I}\end{array}\right]$ and $\mathbf{L}^{-1}=\sqrt{\frac{1}{2}}\left[\begin{array}{cc}\mathbf{I} & \mathbf{I} \\ \mathbf{I} & -\mathbf{I}\end{array}\right]$,

yielding $\exp (-\widehat{\mathbf{A}} \tau)=\mathbf{L} \exp (-\widetilde{\mathbf{A}} \tau) \mathbf{L}^{-1}, \mathbf{D}_{n}(\widehat{\mathbf{A}} \tau)=\mathbf{L D}_{n}(\widetilde{\mathbf{A}} \tau) \mathbf{L}^{-1}$, and $\mathbf{N}_{n}(\widehat{\mathbf{A}} \tau)=\mathbf{L} \mathbf{N}_{n}(\widetilde{\mathbf{A}} \tau) \mathbf{L}^{-1}$. Thus, by this similarity transformation, we have to compute powers of $\widetilde{\mathbf{A}}$, for which we find

$$
\begin{aligned}
\widetilde{\mathbf{A}}^{2 k} & =\left[\begin{array}{cc}
\mathbf{U} & 0 \\
0 & \mathbf{U}^{T}
\end{array}\right]^{k}=\left[\begin{array}{cc}
\mathbf{U}^{k} & 0 \\
0 & \left(\mathbf{U}^{T}\right)^{k}
\end{array}\right], \\
\widetilde{\mathbf{A}}^{2 k+1} & =\widetilde{\mathbf{A}}^{2 k} \widetilde{\mathbf{A}}=\left[\begin{array}{cc}
0 & \mathbf{U}^{k} \widehat{\mathbf{A}}_{-} \\
\left(\mathbf{U}^{T}\right)^{k} \widehat{\mathbf{A}}_{+} & 0
\end{array}\right],
\end{aligned}
$$


Table 2: Relative errors $\varepsilon_{\mathrm{R}}$ and $\varepsilon_{\mathrm{T}}$ for the series approximations (Padé and Taylor approximations with $n=5$ ) and the exponential infinitesimal generator initialization (EIGI).

\begin{tabular}{|c|c|c|c|c|c|}
\hline \multirow[b]{2}{*}{$\bar{\tau}$} & \multirow[b]{2}{*}{$\|\widehat{\mathbf{A}} \bar{\tau}\|_{2}$} & \multicolumn{2}{|c|}{ Series approximations } & \multicolumn{2}{|c|}{ EIGI } \\
\hline & & $\varepsilon_{\mathrm{R}}$ & $\varepsilon_{\mathrm{T}}$ & $\varepsilon_{\mathrm{R}}$ & $\varepsilon_{\mathrm{T}}$ \\
\hline $5.0 \mathrm{e}-4$ & 0.30 & $2.12 \mathrm{e}-3$ & $1.85 \mathrm{e}-3$ & $1.16 \mathrm{e}-2$ & $1.18 \mathrm{e}-2$ \\
\hline $1.0 \mathrm{e}-3$ & 0.66 & $4.38 \mathrm{e}-3$ & $3.87 \mathrm{e}-3$ & $2.15 \mathrm{e}-2$ & $2.29 \mathrm{e}-2$ \\
\hline $2.0 \mathrm{e}-3$ & 1.22 & $9.47 \mathrm{e}-3$ & $1.14 \mathrm{e}-2$ & $3.65 \mathrm{e}-2$ & $4.48 \mathrm{e}-2$ \\
\hline $3.0 \mathrm{e}-3$ & 1.83 & $1.54 \mathrm{e}-2$ & $2.85 \mathrm{e}-2$ & $4.58 \mathrm{e}-2$ & $6.91 \mathrm{e}-2$ \\
\hline $4.0 \mathrm{e}-3$ & 2.44 & $2.23 \mathrm{e}-2$ & $6.15 \mathrm{e}-2$ & $5.12 \mathrm{e}-2$ & $9.79 \mathrm{e}-2$ \\
\hline $5.0 \mathrm{e}-3$ & 3.05 & $2.98 \mathrm{e}-2$ & $9.18 \mathrm{e}-2$ & $5.45 e-2$ & $1.31 \mathrm{e}-1$ \\
\hline
\end{tabular}

where $\mathbf{U}=\widehat{\mathbf{A}}_{-} \widehat{\mathbf{A}}_{+}$, and $\widehat{\mathbf{A}}_{ \pm}=\widehat{\mathbf{A}}_{11} \pm \widehat{\mathbf{A}}_{12}$.

The accuracy of the reflection and transmission matrices computed by the Padé and Taylor series approximations is high if $\|\widehat{\mathbf{A}} \bar{\tau}\| \leq 1$; thus, for small values of $\bar{\tau}$. Therefore, the series approximations can be used for the initialization of the doubling method in radiative transfer. The doubling method is equivalent to the so-called scaling and squaring technique for reducing the norm of a matrix and exploits a fundamental property unique to the exponential function, namely

$\mathrm{e}^{-\widehat{\mathbf{A}} \bar{\tau}}=\left(\mathrm{e}^{-\widehat{\mathbf{A}} \bar{\tau} / 2^{s}}\right)^{2^{s}}$.

In practice, we choose $s$ as the smallest power of 2 for which $\|\widehat{\mathbf{A}} \bar{\tau}\| / 2^{s} \leq 1$, evaluate the reflection and transmission matrices of the layer of optical thickness $\bar{\tau} / 2^{s}$ by means of series approximations, and use the doubling equations to build up the reflection and transmission matrices of the layer of optical thickness $\bar{\tau}$. As compared to other initialization methods based on the single scattering approximation, e.g., the exponential infinitesimal generator initialization (EIGI) given by [41]

$$
\begin{aligned}
& {\left[\widehat{\mathbf{R}}_{\mathrm{EIGI}}\right]_{k l}=\mu_{k}\left(1-\mathrm{e}^{-\frac{1}{\mu_{k}} \bar{\tau}}\right)\left[\widehat{\mathbf{A}}_{12}\right]_{k l},} \\
& {\left[\widehat{\mathbf{T}}_{\mathrm{EIGI}}\right]_{k l}=\mu_{k}\left(1-\mathrm{e}^{-\frac{1}{\mu_{k}} \bar{\tau}}\right)\left[\widehat{\mathbf{A}}_{11}^{0}\right]_{k l}+\delta_{k l} \mathrm{e}^{-\frac{1}{\mu_{k}} \bar{\tau}},}
\end{aligned}
$$

with $\widehat{\mathbf{A}}_{11}^{0}=\widehat{\mathbf{A}}_{11}+\mathbf{M}$ (see (292) below), the series approximations are more accurate and so, reduce the number of doubling needed. This result can be inferred from Table 2, in which the relative errors in the discrete approximations to the reflection and transmission functions for different values of $\|\widehat{\mathbf{A}} \bar{\tau}\|_{2}$ are illustrated. Note that the Padé and Taylor approximations yield the same relative errors, and that the relative errors are of about $1 \%$ for $\|\widehat{\mathbf{A}} \bar{\tau}\|_{2} \leq 1$.

It is a simple exercise to combine the Padé approximation with the Taylor series approximation to derive an approximation of the layer equation in the discrete ordinate method with matrix exponential. Starting from the solution representation (18) and using the Padé approximation for $\exp (-\widehat{\mathbf{A}} \bar{\tau})$ and the Taylor series approximation for $\exp [-\widehat{\mathbf{A}}(\bar{\tau}-\tau)]$, gives the $n$ th-order approximation of the layer equation (compare to (56))

$\mathbf{D}_{n}(\widehat{\mathbf{A}} \bar{\tau}) \widehat{\mathbf{i}}_{\mathrm{d}}(\bar{\tau})=\mathbf{N}_{n}(\widehat{\mathbf{A}} \bar{\tau}) \widehat{\mathbf{i}}_{\mathrm{d}}(0)-\mathbf{B}_{n}(\bar{\tau}) \widehat{\mathbf{b}}$, where

$$
\begin{aligned}
\mathbf{B}_{n}(\bar{\tau}) & =\mathbf{D}_{n}(\widehat{\mathbf{A}} \bar{\tau}) \int_{0}^{\bar{\tau}} \mathrm{e}^{-\widehat{\mathbf{A}}(\bar{\tau}-\tau)} \mathrm{e}^{-\tau / \mu_{0}} \mathrm{~d} \tau \\
& =I_{0}(\bar{\tau}) \mathbf{I}+\sum_{k=1}^{n}\left[c_{k} \bar{\tau}^{k} I_{0}(\bar{\tau})+\frac{(-1)^{k}}{k !} I_{k}(\bar{\tau})\right. \\
& \left.+\sum_{l=1}^{\min (k-1, n)}\left(1-\delta_{k 1}\right) \frac{(-1)^{k-l}}{(k-l) !} c_{l} \bar{\tau}^{l} I_{k-l}(\bar{\tau})\right] \widehat{\mathbf{A}}^{k},
\end{aligned}
$$

and

$$
\begin{aligned}
& I_{0}(\bar{\tau})=\int_{0}^{\bar{\tau}} \mathrm{e}^{-\tau / \mu_{0}} \mathrm{~d} \tau=\frac{1-\mathrm{e}^{-\bar{\tau} / \mu_{0}}}{\left(1 / \mu_{0}\right)} \\
& I_{k}(\bar{\tau})=\int_{0}^{\bar{\tau}}(\bar{\tau}-\tau)^{k} \mathrm{e}^{-\tau / \mu_{0}} \mathrm{~d} \tau^{\prime}=\frac{\bar{\tau}^{k}}{\left(1 / \mu_{0}\right)}-\frac{k \mathrm{e}^{-\bar{\tau} / \mu_{0}}}{\left(1 / \mu_{0}\right)} I_{k-1}(\bar{\tau}), k \geq 1 .
\end{aligned}
$$

Note that because of

$I_{k}(\bar{\tau})=O\left(\frac{\bar{\tau}^{k+1}}{k+1}\right), \bar{\tau}<1$,

each term in the sum (190) behaves as $O\left(\bar{\tau}^{k+1} \widehat{\mathbf{A}}^{k}\right)$. The first-order approximation of (189) is equivalent to the finitedifference method described by Lenoble [5], and used in atmospheric remote sensing by Rozanov et al. [42].

\subsection{Reflection and transmission matrices of a homogeneous thick layer}

When the optical thickness is sufficiently large, the reflection and transmission matrices can be expressed by simple analytical expressions known as the asymptotic theory of thick layers. This analytical model is much faster and more convenient for theoretical considerations than numerical models based on discrete ordinate schemes.

\subsubsection{Asymptotic theory}

In the classical asymptotic theory, the reflection and transmission functions for optically thick atmospheres are given by [32]:

$$
\begin{aligned}
& R_{m}\left(\mu, \mu^{\prime} ; \bar{\tau}\right)=R_{\infty m}\left(\mu, \mu^{\prime}\right)-\delta_{m 0} \frac{\mathrm{ml} \mathrm{e}^{-2 \mathrm{k} \bar{\tau}}}{1-1^{2} \mathrm{e}^{-2 \mathrm{k} \bar{\tau}}} K(\mu) K\left(\mu^{\prime}\right), \\
& T_{m}\left(\mu, \mu^{\prime} ; \bar{\tau}\right)=\delta_{m 0} \frac{\mathrm{me}^{-\mathrm{k} \bar{\tau}}}{1-1^{2} \mathrm{e}^{-2 \mathrm{k} \bar{\tau}}} K(\mu) K\left(\mu^{\prime}\right) .
\end{aligned}
$$

Here, $\mathrm{k}$ is the diffusion exponent describing the attenuation of the radiation in the diffusion domain and being defined as the smallest positive eigenvalue of the equation

$(1-\mathrm{k} \mu) i(\mu)=\frac{\omega}{2} \int_{-1}^{1} p_{0}\left(\mu, \mu^{\prime}\right) i\left(\mu^{\prime}\right) \mathrm{d} \mu^{\prime}$,

while $i(\mu)$ is the corresponding eigenfunction, or the diffusion pattern, satisfying the Sobolev-van de Hulst relation

$i(-\mu)=2 \int_{0}^{1} R_{\infty 0}\left(\mu, \mu^{\prime}\right) i\left(\mu^{\prime}\right) \mu^{\prime} \mathrm{d} \mu^{\prime}$ 
and the normalization condition

$\frac{1}{2} \int_{-1}^{1} i(\mu) \mathrm{d} \mu=1$

From (195), (197), and the normalization condition of the phase functions $\int_{-1}^{1} p_{0}\left(\mu, \mu^{\prime}\right) \mathrm{d} \mu^{\prime}=2$, we find the following expression for the diffusion exponent,

$\mathrm{k}=\frac{2(1-\omega)}{\int_{-1}^{1} i(\mu) \mu \mathrm{d} \mu}$

The escape function $K(\mu)$ is given by the relation

$\mathrm{m} K(\mu)=i(\mu)-2 \int_{0}^{1} R_{\infty 0}\left(\mu, \mu^{\prime}\right) i\left(-\mu^{\prime}\right) \mu^{\prime} \mathrm{d} \mu^{\prime}$

and satisfies the normalization condition

$2 \int_{0}^{1} K(\mu) i(\mu) \mu \mathrm{d} \mu=1$

where the constant $m$ is defined by

$\mathrm{m}=2 \int_{-1}^{1} i^{2}(\mu) \mu \mathrm{d} \mu$

Finally, the constant 1 , also known as the negative internal reflection coefficient, is computed as

$1=2 \int_{0}^{1} K(\mu) i(-\mu) \mu \mathrm{d} \mu$.

Relations (193) and (194) show that $R$ depends on the azimuthal angle through the reflection function of a semiinfinite atmosphere $R_{\infty}$, and that $T$ is azimuthally independent. For a layer with an underlying Lambertian surface of albedo $A$, the reflection and transmission functions $R_{A m}\left(\mu, \mu^{\prime} ; \bar{\tau}\right)$ and $T_{A m}\left(\mu, \mu^{\prime} ; \bar{\tau}\right)$ are given by (82) and (83), respectively, with

$$
\begin{aligned}
& r(\mu, \bar{\tau})=r_{\infty}(\mu)-\frac{\mathrm{mnl}}{1-1^{2} \mathrm{e}^{-2 \mathrm{k} \bar{\tau}}} \mathrm{e}^{-2 \mathrm{k} \bar{\tau}} K(\mu), \\
& t(\mu, \bar{\tau})=\frac{\mathrm{mn}}{1-1^{2} \mathrm{e}^{-2 \mathrm{k} \bar{\tau}}} \mathrm{e}^{-\mathrm{k} \bar{\tau}} K(\mu), \\
& r_{s}(\bar{\tau})=r_{s \infty}-\frac{\mathrm{mn}^{2} 1}{1-1^{2} \mathrm{e}^{-2 \mathrm{k} \bar{\tau}}} \mathrm{e}^{-2 \mathrm{k} \bar{\tau}} .
\end{aligned}
$$

Here, $r_{\infty}$ and $r_{s \infty}$ are the plane albedo and spherical albedo of a semi-infinite atmosphere, respectively, and $\mathrm{n}$ is the $\mu$ weighted mean of the escape function

$\mathrm{n}=2 \int_{0}^{1} K(\mu) \mu \mathrm{d} \mu$.

In a discrete ordinate setting, the reflection matrix of a semi-infinite atmosphere can be obtained by solving the Ambartsumian nonlinear integral equation by simple iteration [35], while the diffusion pattern and the diffusion exponent can be obtained by solving the integral equation (195) in conjunction with (197) and (198) [43]. The constant $m$ is then computed from (201), while $K(\mu), 1$, and $\mathrm{n}$ follow from (199), (202), and (206), respectively. A different discrete ordinate approach, which is based on an eigendecomposition method for computing the reflection and transmission matrices has been proposed by Nakajima and King [27]. Here, we apply this approach to estimate $\widehat{\mathbf{R}}$ and $\widehat{\mathbf{T}}$, given respectively by (97) and (98), in the limit of large $\bar{\tau}$. Choosing the squareroot method for computing the spectral decomposition of the layer matrix $\widehat{\mathbf{A}}$, we begin by estimating the matrix product $\left(\widehat{\mathbf{V}}_{+}+\widehat{\mathbf{V}}_{-} \boldsymbol{\Gamma}\right)\left(\widehat{\mathbf{V}}_{-}+\widehat{\mathbf{V}}_{+} \boldsymbol{\Gamma}\right)^{-1}$ for large values of the optical thickness. Using the identity (cf. (344)) $\widehat{\mathbf{Q}}_{-}=-\widehat{\mathbf{Q}}_{+}^{-T} \boldsymbol{\Lambda}$, where $\widehat{\mathbf{Q}}_{+}=\widehat{\mathbf{V}}_{+}+\widehat{\mathbf{V}}_{-}, \widehat{\mathbf{Q}}_{-}=\widehat{\mathbf{V}}_{+}-\widehat{\mathbf{V}}_{-}$, and $\boldsymbol{\Lambda}=\left[\lambda_{k}\right]$, and setting

$\widehat{\mathbf{Q}}_{+}^{-T} \stackrel{\text { not }}{=} \mathbf{Q}=\left[\mathbf{q}_{1}, \ldots, \mathbf{q}_{N}\right]$

we obtain

$\left(\widehat{\mathbf{V}}_{+}+\widehat{\mathbf{V}}_{-} \boldsymbol{\Gamma}\right)\left(\widehat{\mathbf{V}}_{-}+\widehat{\mathbf{V}}_{+} \boldsymbol{\Gamma}\right)^{-1}$

$=\left(\mathbb{A}^{-1}-\mathbf{I}\right)+\mathbb{A}^{-1} \mathbf{Q}\left[\boldsymbol{\Lambda}\left(\mathbf{I}-\boldsymbol{\Pi}_{+} \mathbb{B} \boldsymbol{\Lambda}\right)^{-1} \boldsymbol{\Pi}_{+}\right] \mathbf{Q}^{T} \mathbb{A}^{-1}$,

where

$\mathbb{A}=\frac{1}{2}\left(\mathbf{I}+\mathbf{Q} \mathbf{\Lambda} \mathbf{Q}^{T}\right)$,

$\mathbb{B}=\mathbf{Q}^{T} \mathbb{A}^{-1} \mathbf{Q}$

and

$\mathbf{\Pi}_{+}=\frac{1}{2}\left[\mathbf{I}-(\mathbf{I}-\boldsymbol{\Gamma})(\mathbf{I}+\mathbf{\Gamma})^{-1}\right]=\left[\pi_{k}^{+}\right]$,

$\pi_{k}^{+}=\frac{\mathrm{e}^{-\lambda_{k} \bar{\tau}}}{1+\mathrm{e}^{-\lambda_{k} \bar{\tau}}}, k=1, \ldots, N$.

Note that all information about the eigenvectors of $\widehat{\mathbf{A}}$ are encapsulated in the matrix $\mathbf{Q}$. Two comments are in order:

1. As $\mathbb{A}$ and $\mathbb{B}$ are symmetric, we see that

$\boldsymbol{\Lambda}\left(\mathbf{I}-\boldsymbol{\Pi}_{+} \mathbb{B} \boldsymbol{\Lambda}\right)^{-1} \boldsymbol{\Pi}_{+}=\left[\left(\boldsymbol{\Lambda} \boldsymbol{\Pi}_{+}\right)^{-1}-\mathbb{B}\right]^{-1}$

and so, that $\left(\widehat{\mathbf{V}}_{+}+\widehat{\mathbf{V}}_{-} \boldsymbol{\Gamma}\right)\left(\widehat{\mathbf{V}}_{-}+\widehat{\mathbf{V}}_{+} \boldsymbol{\Gamma}\right)^{-1}$ are symmetric.

2. If the eigendecomposition of $\widehat{\mathbf{A}}$ is computed by the Cholesky method, the key quantity is the matrix $\widehat{\mathbf{Q}}_{-}$; we use the identity (cf. (351)) $\widehat{\mathbf{Q}}_{+}^{-T}=-\widehat{\mathbf{Q}}_{-} \boldsymbol{\Lambda}$, set

$\widehat{\mathbf{Q}}_{-} \stackrel{\text { not }}{=} \mathbf{Q}=\left[\mathbf{q}_{1}, \ldots, \mathbf{q}_{N}\right]$

and obtain

$$
\begin{aligned}
& \left(\widehat{\mathbf{V}}_{+}+\widehat{\mathbf{V}}_{-} \boldsymbol{\Gamma}\right)\left(\widehat{\mathbf{V}}_{-}+\widehat{\mathbf{V}}_{+} \boldsymbol{\Gamma}\right)^{-1} \\
& =\left(\mathbb{A}^{-1}-\mathbf{I}\right)+\mathbb{A}^{-1} \mathbf{Q}\left[\left(\mathbf{I}-\boldsymbol{\Pi}_{+} \boldsymbol{\Lambda} \mathbb{B}\right)^{-1} \boldsymbol{\Pi}_{+} \boldsymbol{\Lambda}\right] \mathbf{Q}^{T} \mathbb{A}^{-1}
\end{aligned}
$$

Then, from (213) and the relation

$\left(\mathbf{I}-\boldsymbol{\Pi}_{+} \boldsymbol{\Lambda} \mathbb{B}\right)^{-1} \boldsymbol{\Pi}_{+} \boldsymbol{\Lambda}=\left[\left(\boldsymbol{\Lambda} \boldsymbol{\Pi}_{+}\right)^{-1}-\mathbb{B}\right]^{-1}$,

we deduce that both methods are equivalent. 
Considering (211) and (212), we assume that for large $\bar{\tau}$, we may approximate

$\boldsymbol{\Pi}_{+} \approx \pi_{N}^{+}\left[\begin{array}{ccc}0 & \cdots & 0 \\ 0 & \cdots & 1\end{array}\right]$,

where $\pi_{N}^{+}$corresponds to the smallest eigenvalue $\lambda_{N}$ in the set $\left\{\lambda_{1}, \lambda_{2}, \ldots, \lambda_{N}\right\}$. As a result, we obtain

$\left(\widehat{\mathbf{V}}_{+}+\widehat{\mathbf{V}}_{-} \boldsymbol{\Gamma}\right)\left(\widehat{\mathbf{V}}_{-}+\widehat{\mathbf{V}}_{+} \boldsymbol{\Gamma}\right)^{-1}$

$\approx\left(\mathbb{A}^{-1}-\mathbf{I}\right)+\frac{\mathrm{ke}^{-\mathrm{k} \bar{\tau}}}{1+1 \mathrm{e}^{-\mathrm{k} \bar{\tau}}} \widehat{\mathbf{k}}_{N} \widehat{\mathbf{k}}_{N}^{T}$ as $\tau \rightarrow \infty$,

where $\mathrm{k}=\lambda_{N}$ is the diffusion exponent,

$1=1-\lambda_{N} b_{N N}=1-\mathrm{k} q$,

is the negative internal reflection coefficient, $q=b_{N N}=$ $[\mathbb{B}]_{N N}$ is the extrapolation length, and

$\widehat{\mathbf{k}}_{N}=\mathbb{A}^{-1} \mathbf{q}_{N}$,

is a discrete approximation to the scaled escape function. The matrix product $\left(\widehat{\mathbf{V}}_{+}-\widehat{\mathbf{V}}_{-} \boldsymbol{\Gamma}\right)\left(\widehat{\mathbf{V}}_{-}-\widehat{\mathbf{V}}_{+} \boldsymbol{\Gamma}\right)^{-1}$ is estimated in a similar manner. We get

$\left(\widehat{\mathbf{V}}_{+}-\widehat{\mathbf{V}}_{-} \boldsymbol{\Gamma}\right)\left(\widehat{\mathbf{V}}_{-}-\widehat{\mathbf{V}}_{+} \boldsymbol{\Gamma}\right)^{-1}$

$=\left(\mathbb{A}^{-1}-\mathbf{I}\right)+\mathbb{A}^{-1} \mathbf{Q}\left[\boldsymbol{\Lambda}\left(\mathbf{I}-\boldsymbol{\Pi}_{-} \mathbb{B} \boldsymbol{\Lambda}\right)^{-1} \boldsymbol{\Pi}_{-}\right] \mathbf{Q}^{T} \mathbb{A}^{-1}$,

with

$\mathbf{\Pi}_{-}=\frac{1}{2}\left[\mathbf{I}-(\mathbf{I}+\boldsymbol{\Gamma})(\mathbf{I}-\boldsymbol{\Gamma})^{-1}\right]=\left[\pi_{k}^{-}\right]$,

$\pi_{k}^{-}=-\frac{\mathrm{e}^{-\lambda_{k} \bar{\tau}}}{1-\mathrm{e}^{-\lambda_{k} \bar{\tau}}}, k=1, \ldots, N$,

whence, under the assumption

$\boldsymbol{\Pi}_{-} \approx \pi_{N}^{-}\left[\begin{array}{ccc}0 & \cdots & 0 \\ 0 & \cdots & 1\end{array}\right]$ as $\tau \rightarrow \infty$,

we end up with

$\left(\widehat{\mathbf{V}}_{+}-\widehat{\mathbf{V}}_{-} \boldsymbol{\Gamma}\right)\left(\widehat{\mathbf{V}}_{-}-\widehat{\mathbf{V}}_{+} \boldsymbol{\Gamma}\right)^{-1}$

$\approx\left(\mathbb{A}^{-1}-\mathbf{I}\right)-\frac{\mathrm{ke}^{-\mathrm{k} \bar{\tau}}}{1-1 \mathrm{e}^{-\mathrm{k} \bar{\tau}}} \widehat{\mathbf{k}}_{N} \widehat{\mathbf{k}}_{N}^{T}$ as $\tau \rightarrow \infty$.

Inserting (218) and (225) in (97) and (98), we obtain

$\widehat{\mathbf{R}}=\widehat{\mathbf{R}}_{\infty}-\widehat{\mathbf{r}} \widehat{\mathbf{k}}_{N} \widehat{\mathbf{k}}_{N}^{T}$,

$\widehat{\mathbf{T}}=\widehat{\mathrm{t}} \widehat{\mathbf{k}}_{N} \widehat{\mathbf{k}}_{N}^{T}$,

where

$\widehat{\mathbf{R}}_{\infty}=\mathbb{A}^{-1}-\mathbf{I}$,

is the scaled reflection matrix of a semi-infinite atmosphere, and

$\widehat{\mathrm{r}}=\frac{\mathrm{k} l \mathrm{e}^{-2 \mathrm{k} \bar{\tau}}}{1-\mathrm{l}^{2} \mathrm{e}^{-2 \mathrm{k} \bar{\tau}}}$

$\widehat{\mathrm{t}}=\frac{\mathrm{ke}^{-\mathrm{k} \bar{\tau}}}{1-1^{2} \mathrm{e}^{-2 \mathrm{k} \bar{\tau}}}$.

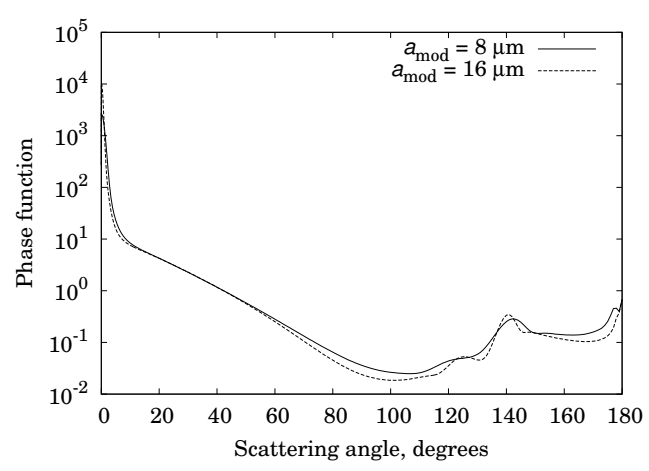

Figure 1: The phase functions for $a_{\mathrm{mod}}=8 \mu \mathrm{m}$ and $a_{\mathrm{mod}}=16 \mu \mathrm{m}$.

By numerical simulation it can be shown that the diffusion exponent $\mathrm{k}=\lambda_{N}$ increases as the azimuthal mode $m$ increases. In this limit, the scalars $\widehat{r}$ and $\widehat{t}$ become very small, and the contributions of the terms $\widehat{\mathbf{r}} \widehat{\mathbf{k}}_{N} \widehat{\mathbf{k}}_{N}^{T}$ and $\widehat{\mathrm{t}} \widehat{\mathbf{k}}_{N} \widehat{\mathbf{k}}_{N}^{T}$ in the expressions of $\widehat{\mathbf{R}}$ and $\widehat{\mathbf{T}}$, respectively, are damped. In the asymptotic theory, these terms are neglected for $m>0$, in which case, (226) and (227) simplifies to

$\widehat{\mathbf{R}}=\widehat{\mathbf{R}}_{\infty}-\delta_{m 0} \widehat{\mathbf{r}} \widehat{\mathbf{k}}_{N} \widehat{\mathbf{k}}_{N}^{T}$,

$\widehat{\mathbf{T}}=\delta_{m 0} \widehat{\mathrm{t}} \widehat{\mathbf{k}}_{N} \widehat{\mathbf{k}}_{N}^{T}$.

Thus, the diffusion exponent $\mathrm{k}$ and the constant 1 in (193) and (194) correspond to the azimuthal mode $m=0$. As a final step, we compute the discrete approximations of the reflection and transmission functions given by (193) and (194), respectively, by means of (117) and (118), respectively. The remainder functions and constants that occur in the asymptotic theory can be obtained by following strictly the derivation of Nakajima and King [27]. Their expressions are given in Appendix 1 .

As an application of the discrete ordinate model of the asymptotic theory we derive parameterizations of the escape function $K$ and the asymptotic constants $\mathrm{m}, 1, \mathrm{n}, r_{s \infty}$ and $\mathrm{k}$. Such kind of parameterizations, which speed up the computations, are used in the MODIS algorithm for the retrieval of cloud optical thickness and the droplet/crystal size [44], and in the SemiAnalytical CloUd Retrieval Algorithm (SACURA) for the retrieval of cloud top height and cloud geometrical thickness from measurements in the oxygen A band [45, 46]. For the EPIC instrument, we consider a discrete set of watercloud models characterized by a Gamma size distribution of parameter $\alpha=6$ and different modal radii $a_{\text {mod. }}$. The phase functions for $a_{\mathrm{mod}}=8 \mu \mathrm{m}$ and $a_{\mathrm{mod}}=16 \mu \mathrm{m}$ are illustrated in Figure 1.

For the escape function, the parameterization parameters are $\omega$ and $a_{\text {mod. }}$. We look for polynomial parameterizations of the form $K\left(\mu, \omega, a_{\text {mod }}\right)=P_{\mathrm{K}}\left(\mu, \omega, a_{\text {mod }}\right) K_{0}(\mu)$ with $P_{\mathrm{K}}\left(\mu, \omega, a_{\text {mod }}\right)=\sum_{k=0}^{N_{\mathrm{K}}} H_{k}\left(\omega, a_{\mathrm{mod}}\right) \mu^{k}$, and $K_{0}(\mu)=\sum_{k=0}^{N_{\mathrm{K}}} H_{k}^{0} \mu^{k}$, where $K_{0}$ is the escape function for conservative scattering ( $\omega=1)$, and $N_{\mathrm{K}}$ is the order of the approximation polynomial. The parameterizations of $K\left(\mu, \omega, a_{\bmod }\right)$ do not change significantly with $a_{\text {mod }}$; the coefficients $H_{k}\left(\omega, a_{\text {mod }}\right)$, which are stored in a look-up table, are illustrated in Figure 2 for 
Table 3: Coefficients $H_{k}^{0}$

\begin{tabular}{llllll}
\hline$k$ & 0 & 1 & 2 & 3 & 4 \\
\hline$H_{k}^{0}$ & 0.362 & 1.196 & -0.5352 & 0.349 & -0.09368 \\
\hline
\end{tabular}

$a_{\text {mod }}=8 \mu \mathrm{m}$, and $a_{\text {mod }}=16 \mu \mathrm{m}$, while the coefficients $H_{k}^{0}$ are given in Table 3. The approximations error is smaller than $10^{-3}$ for $K\left(\mu, \omega, a_{\text {mod }}\right)$, and smaller than $10^{-5}$ for $K_{0}(\mu)$. Coming to the asymptotic constants $\mathrm{m}, 1, \mathrm{n}, r_{s \infty}$ and $\mathrm{k}$, we note that for a Henyey-Greenstein phase function, King [47] derived parameterizations of these quantities in terms of the similarity parameter

$s=\sqrt{\frac{1-\omega}{1-\omega g}}$

where $g$ is the asymmetry parameter. In this regard, we take $s$ and $a_{\text {mod }}$ as parameterization parameters ( $s$ reproduces the variability in $\omega$ for a given $a_{\text {mod }}$ ). As for the escape function, we found that the parameterizations of the asymptotic constant are almost insensitive to $a_{\text {mod }}$, but are slightly different from those of King [47], especially for $\mathrm{m}, \mathrm{n}$, and $\mathrm{k}$. The results in Figure 3, corresponding to $a_{\mathrm{mod}}=8 \mu \mathrm{m}(g=0.853)$ and $a_{\mathrm{mod}}=16 \mu \mathrm{m}(\mathrm{g}=0.867)$, certify this statement. For $a_{\mathrm{mod}}=8$ $\mu \mathrm{m}$, the parameterizations read as

$\mathrm{m}(s)=(1+1.268 s) \ln \left[\frac{1+1.31 s-5.694 s^{2}+3.73 s^{3}}{(1-1.070 s)(1-s)^{2}}\right]$

$1(s)=\frac{(1-0.7616 s)(1-s)}{1+0.5897 s}$

$\mathrm{n}(s)=\sqrt{\frac{(1+0.0031 s)(1-s)}{1+1.4267 s}}$,

$r_{s \infty}(s)=\frac{(1-0.1239 s)(1-s)}{1+1.1867 s}$

$\frac{\mathrm{k}(s)}{1-g}=(1+0.3977 s) \ln \left[\frac{1+8.4924 s}{(1+7.2543 s)(1-1.0265 s)}\right]$.

\subsubsection{Higher-order corrections}

The azimuthal independent parts of $\widehat{\mathbf{R}}$ and $\widehat{\mathbf{T}}$ have been derived by neglecting the azimuthal modes $m>0$, and the contributions of the terms corresponding to the eigenvalues larger than $\lambda_{N}$. Higher-order corrections can be obtained by considering all azimuthal modes, and by approximating

$\Pi_{ \pm} \approx\left[\begin{array}{cccccc}0 & \cdots & 0 & \cdots & 0 & 0 \\ 0 & \cdots & \pi_{N-K}^{ \pm} & \cdots & 0 & 0 \\ 0 & \cdots & 0 & \cdots & \pi_{N-1}^{ \pm} & 0 \\ 0 & \cdots & 0 & \cdots & 0 & \pi_{N}^{ \pm}\end{array}\right]$

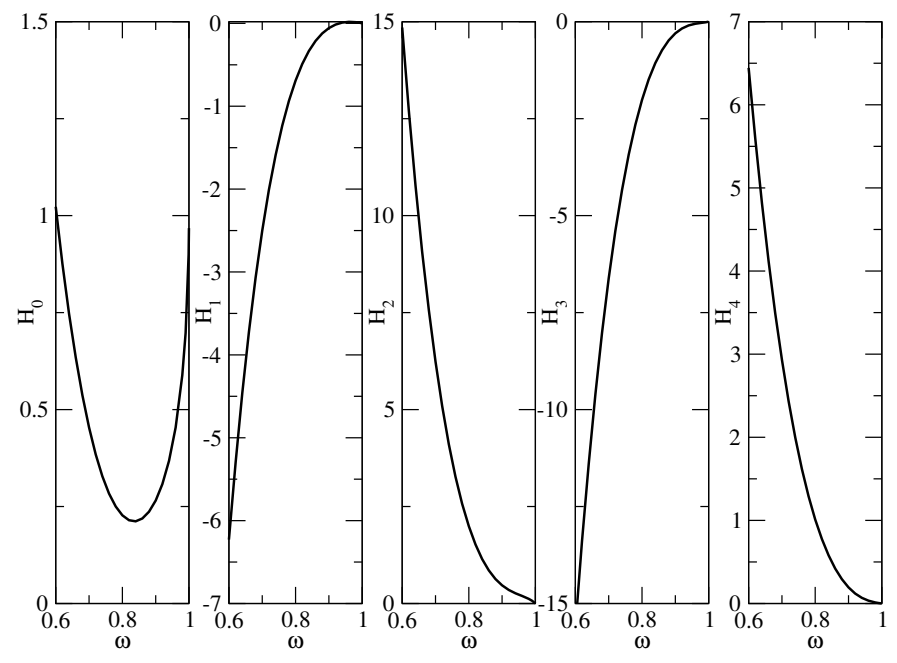

Figure 2: Coefficients $H_{k}\left(\omega, a_{\mathrm{mod}}\right)$ for $a_{\mathrm{mod}}=8 \mu \mathrm{m}$ and $a_{\mathrm{mod}}=16 \mu \mathrm{m}$.

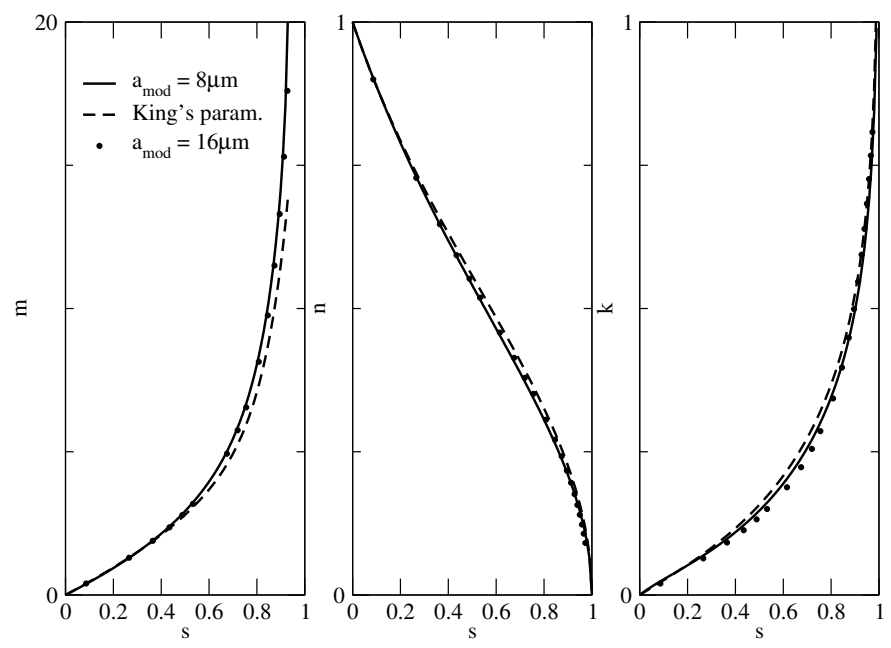

Figure 3: Constants $\mathrm{m}$ and $\mathrm{n}$, and the diffusion exponent $\mathrm{k}$. The curves correspond to $a_{\mathrm{mod}}=8 \mu \mathrm{m}, a_{\mathrm{mod}}=16 \mu \mathrm{m}$, and King's parametrization.

where $\pi_{k}^{+}$and $\pi_{k}^{-}$are given by (212) and (223), respectively, and the integer $K \geq 0$ (not to be confused with the escape function $K(\mu)$ ) gives the order of the approximation. For $\boldsymbol{\Pi}_{ \pm}$ of (233), the inverse $\left(\mathbf{I}-\boldsymbol{\Pi}_{ \pm} \mathbb{B} \boldsymbol{\Lambda}\right)^{-1}$ cannot be computed analytically, and an additional assumption has to be met. Setting

$\mathbf{I}-\boldsymbol{\Pi}_{ \pm} \mathbb{B} \boldsymbol{\Lambda}=\mathbb{C}_{ \pm}^{0}-\mathbb{C}_{ \pm}$,

where $\mathbb{C}_{ \pm}^{0}$ are the matrices $\mathbf{I}-\boldsymbol{\Pi}_{ \pm} \mathbb{B} \boldsymbol{\Lambda}$ of the asymptotic model (with $\boldsymbol{\Pi}_{+}$and $\boldsymbol{\Pi}_{-}$given by (217) and (224), respectively), we approximate the Neumann series of the inverse up to the first order as follows:

$\left(\mathbf{I}-\boldsymbol{\Pi}_{ \pm} \mathbb{B} \boldsymbol{\Lambda}\right)^{-1}=\left(\mathbb{C}_{ \pm}^{0}-\mathbb{C}_{ \pm}\right)^{-1} \approx\left(\mathbb{C}_{ \pm}^{0}\right)^{-1}\left[\mathbf{I}+\mathbb{C}_{ \pm}\left(\mathbb{C}_{ \pm}^{0}\right)^{-1}\right]$.

In this context, the reflection and transmission matrices can be written as

$\mathbb{R}=\mathbb{R}_{0}+\mathbb{R}_{K}$

$\mathbb{T}=\mathbb{T}_{0}+\mathbb{T}_{K}$, 
where $\mathbb{R}_{0}$ and $\mathbb{t}_{0}$ correspond to $K=0$ and are the reflection and transmission matrices of the asymptotic model as given by (231) and (232), respectively. The higher-order correction matrices $\mathbb{R}_{K}$ and $\mathbb{\mathbb { T }}_{K}$ correspond to $K \geq 1$, and are expressed in terms of the scaled matrices

$$
\begin{aligned}
\widehat{\mathbf{R}}_{K} & =\frac{1}{2} \lambda_{N} \mathrm{e}^{-\lambda_{N} \bar{\tau}}\left(\frac{1}{1+1 \mathrm{e}^{-\lambda_{N} \bar{\tau}}} \mathbb{E}_{N-K}^{+}-\frac{1}{1-1 \mathrm{e}^{-\lambda_{N} \bar{\tau}}} \mathbb{E}_{N-K}^{-}\right) \\
& +\frac{1}{2} \lambda_{N} \sum_{k=1}^{K} \mathrm{e}^{-\lambda_{N-k} \bar{\tau}}\left(\frac{1}{1+\mathrm{e}^{-\lambda_{N-k} \bar{\tau}}} \mathbb{F}_{N-k}^{+}-\frac{1}{1-\mathrm{e}^{-\lambda_{N-k} \bar{\tau}}} \mathbb{F}_{N-k}^{-}\right),
\end{aligned}
$$

$$
\begin{aligned}
\widehat{\mathbf{T}}_{K} & =\frac{1}{2} \lambda_{N} \mathrm{e}^{-\lambda_{N} \bar{\tau}}\left(\frac{1}{1+1 \mathrm{e}^{-\lambda_{N} \bar{\tau}}} \mathbb{E}_{N-K}^{+}+\frac{1}{1-1 \mathrm{e}^{-\lambda_{N} \bar{\tau}}} \mathbb{E}_{N-K}^{-}\right) \\
& +\frac{1}{2} \lambda_{N} \sum_{k=1}^{K} \mathrm{e}^{-\lambda_{N-k} \bar{\tau}}\left(\frac{1}{1+\mathrm{e}^{-\lambda_{N-k} \bar{\tau}}} \mathbb{F}_{N-k}^{+}+\frac{1}{1-\mathrm{e}^{-\lambda_{N-k} \bar{\tau}}} \mathbb{F}_{N-k}^{-}\right),
\end{aligned}
$$

as follows:

$$
\begin{aligned}
& \mathbb{R}_{K}=\frac{1}{2} \mathbf{M}^{\frac{1}{2}} \mathbf{W}^{-\frac{1}{2}} \widehat{\mathbf{R}}_{K} \mathbf{W}^{-\frac{1}{2}} \mathbf{M}^{\frac{1}{2}}, \\
& \mathbb{T}_{K}=\frac{1}{2} \mathbf{M}^{\frac{1}{2}} \mathbf{W}^{-\frac{1}{2}} \widehat{\mathbf{T}}_{K} \mathbf{W}^{-\frac{1}{2}} \mathbf{M}^{\frac{1}{2}} .
\end{aligned}
$$

The derivation of (236) and (237) is lengthy but straightforward. Here, we give only the final expressions. The matrices $\mathbb{E}_{N-K}^{+}$and $\mathbb{F}_{N-k}^{+}$, corresponding to $\Pi_{+}$, are computed as

$\mathbb{E}_{N-K}^{+}=E_{N N}^{+} \widehat{\mathbf{k}}_{N} \widehat{\mathbf{k}}_{N}^{T}+\sum_{k=1}^{K} E_{N-k N}^{+} \widehat{\mathbf{k}}_{N-k} \widehat{\mathbf{k}}_{N}^{T}$

and

$\mathbb{F}_{N-k}^{+}=F_{N N-k}^{+} \widehat{\mathbf{k}}_{N} \widehat{\mathbf{k}}_{N-k}^{T}+\sum_{l=1}^{K} F_{N-l N-k}^{+} \widehat{\mathbf{k}}_{N-l} \widehat{\mathbf{k}}_{N-k}^{T}$,

respectively, with

$\widehat{\mathbf{k}}_{N-k}=\mathrm{A}^{-1} \mathbf{q}_{N-k}$,

for $k=1, \ldots, K$. The scalars $E_{N-k N}^{+}, E_{N N}^{+}, F_{N-l N-k}^{+}$, and $F_{N N-k}^{+}$are

$$
\begin{aligned}
E_{N-k N}^{+} & =f_{N}^{+} g_{N-k} X_{N-k N}^{+}, \\
E_{N N}^{+} & =f_{N}^{+} \sum_{k=1}^{K} x_{N-k}^{+} X_{N-k N}^{+},
\end{aligned}
$$

and

$$
\begin{aligned}
F_{N-l N-k}^{+} & =g_{N-l} X_{N-l N-k}^{+}, \\
F_{N N-k}^{+} & =\sum_{l=1}^{K} x_{N-l}^{+} X_{N-l N-k}^{+},
\end{aligned}
$$

respectively, where

$$
\begin{aligned}
X_{N-k N-l}^{+} & =\delta_{N-k N-l}+Y_{N-k N-l}^{+}, \\
X_{N-k N}^{+} & =\delta_{N-k N}+Y_{N-k N}^{+},
\end{aligned}
$$

and

$$
\begin{aligned}
Y_{N-k N-l}^{+} & =\pi_{N-k}^{+} b_{N-k N-l} \lambda_{N-l}+x_{N-l}^{+} \pi_{N-k}^{+} b_{N-k N} \lambda_{N}, \\
Y_{N-k N}^{+} & =\frac{\pi_{N-k}^{+} b_{N-k N} \lambda_{N}}{f_{N}^{+}},
\end{aligned}
$$

for $k, l=1, \ldots, K$. In (243)-(246), $\pi_{N-k}^{+}$are given by (212), and

$$
\begin{aligned}
x_{N-k}^{+} & =\frac{\pi_{N}^{+} b_{N N-k} \lambda_{N-k}}{f_{N}^{+}}, \\
f_{N}^{+} & =1-\pi_{N}^{+} b_{N N} \lambda_{N}, \\
g_{N-k} & =\frac{\lambda_{N-k}}{\lambda_{N}},
\end{aligned}
$$

for $k=1, \ldots, K$. The matrices $\mathbb{E}_{N-K}^{-}$and $\mathbb{F}_{N-k}^{-}$, corresponding to $\boldsymbol{\Pi}_{-}$, are computed by using (240)-(247), but with $\pi_{N-k}^{-}$of (223) in place of $\pi_{N-k}^{+}$.

Although the discrete ordinate model of the asymptotic theory is still based on an eigendecomposition method, the computation of the reflection and transmission matrices by means of (226), (227), (236), and (237) avoids the matrix inversions and matrix multiplications of (97) and (98).

\subsubsection{Waterman's approximation}

In [21], Waterman derived analytical solutions for the transmission and reflection matrices in the limiting case $\bar{\tau} \gg$ 1. The starting point of Waterman's derivation are (108) and (109), which we will write as

$\widehat{\mathbf{T}}=\widehat{\mathbf{T}}_{0}\left(\mathbf{I}-\widehat{\mathbf{V}}_{+} \boldsymbol{\Gamma} \overline{\mathbf{V}}_{+}^{T} \overline{\mathbf{V}}_{-}^{-T} \boldsymbol{\Gamma} \widehat{\mathbf{V}}_{-}^{-1}\right)^{-1}$,

$\widehat{\mathbf{R}}=\left(\widehat{\mathbf{V}}_{+} \boldsymbol{\Gamma}^{-1} \overline{\mathbf{V}}_{-}^{T}-\widehat{\mathbf{V}}_{-} \boldsymbol{\Gamma} \overline{\mathbf{V}}_{+}^{T}\right) \widehat{\mathbf{T}}$,

with

$\widehat{\mathbf{T}}_{0}=\overline{\mathbf{V}}_{-}^{-T} \boldsymbol{\Gamma} \widehat{\mathbf{V}}_{-}^{-1}$.

Considering the Neuman series for the inverse in (248), we obtain

$\widehat{\mathbf{T}}=\widehat{\mathbf{T}}_{0}\left[\mathbf{I}+\widehat{\mathbf{V}}_{+} \boldsymbol{\Gamma} \overline{\mathbf{V}}_{+}^{T} \overline{\mathbf{V}}_{-}^{-T} \boldsymbol{\Gamma} \widehat{\mathbf{V}}_{-}^{-1}+\ldots\right]$,

$\widehat{\mathbf{R}}=\left(\widehat{\mathbf{V}}_{+} \boldsymbol{\Gamma}^{-1} \overline{\mathbf{V}}_{-}^{T}-\widehat{\mathbf{V}}_{-} \boldsymbol{\Gamma} \overline{\mathbf{V}}_{+}^{T}\right) \widehat{\mathbf{T}}$.

Accounting of (50), we set (not to be confused with $\mathbb{A}$ of (209))

$\mathbb{A}=\overline{\mathbf{V}}_{+}^{T} \overline{\mathbf{V}}_{-}^{-T}=\widehat{\mathbf{V}}_{-}^{-1} \widehat{\mathbf{V}}_{+}$

and use the identity (cf. (50))

$\widehat{\mathbf{V}}_{+} \widehat{\mathbf{V}}_{-}^{-1} \widehat{\mathbf{V}}_{+}=\widehat{\mathbf{V}}_{-}-\overline{\mathbf{V}}_{-}^{-T}$

to obtain the zeroth- and first-order approximations, namely

$\widehat{\mathbf{T}}_{0}=\overline{\mathbf{V}}_{-}^{-T} \boldsymbol{\Gamma} \widehat{\mathbf{V}}_{-}^{-1}$,

$\widehat{\mathbf{R}}_{0}=\widehat{\mathbf{V}}_{+} \widehat{\mathbf{V}}_{-}^{-1}$, 

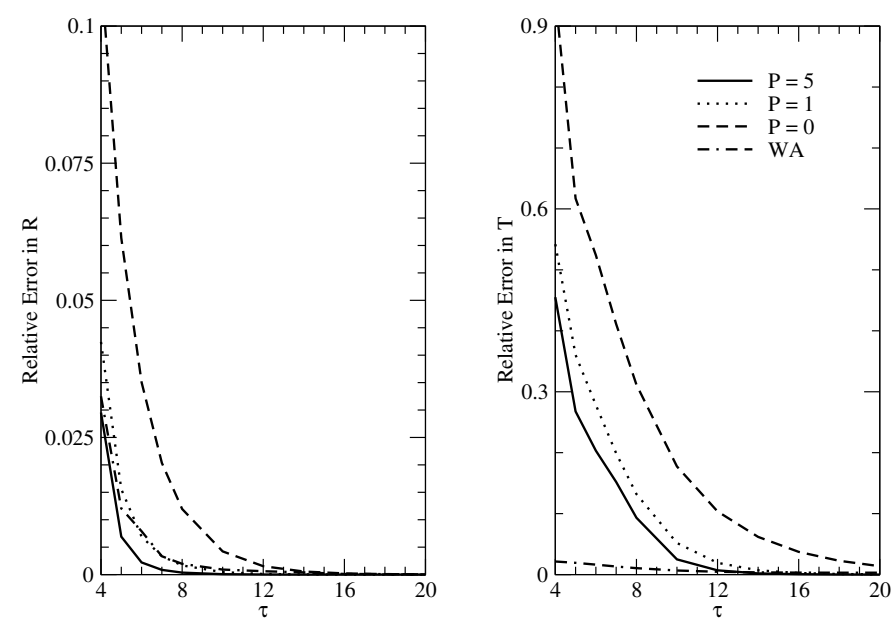

Figure 4: Relative errors in the discrete approximations to the reflection and transmission functions computed by using (234)-(237) with $K=5, K=1$, and $K=0$ (asymptotic model), and the first-order Waterman's approximation (WA) given by (257)-(258).

and

$$
\begin{aligned}
& \widehat{\mathbf{T}}_{1}=\overline{\mathbf{V}}_{-}^{-T} \boldsymbol{\Gamma} \widehat{\mathbf{V}}_{-}^{-1}+\overline{\mathbf{V}}_{-}^{-T}(\boldsymbol{\Gamma} \mathbb{\Gamma} \boldsymbol{\Gamma} \mathbb{\Gamma} \boldsymbol{\Gamma}) \widehat{\mathbf{V}}_{-}^{-1} \\
& \widehat{\mathbf{R}}_{1}=\widehat{\mathbf{V}}_{+} \widehat{\mathbf{V}}_{-}^{-1}-\overline{\mathbf{V}}_{-}^{-T}(\boldsymbol{\Gamma} \mathbb{\Gamma} \boldsymbol{\Gamma}) \widehat{\mathbf{V}}_{-}^{-1}-\widehat{\mathbf{V}}_{-}(\boldsymbol{\Gamma} \mathbb{A} \boldsymbol{\Gamma} \mathbb{\Gamma} \boldsymbol{\Gamma} \boldsymbol{\Gamma}) \widehat{\mathbf{V}}_{-}^{-1}
\end{aligned}
$$

respectively. Note that in Waterman's derivation, the last term in the expression of $\widehat{\mathbf{R}}_{1}$ is not present. In (255)-(258), $\Gamma$ includes all exponential terms $\exp \left(-\lambda_{k} \bar{\tau}\right)$ and not only the dominant one $\exp \left(-\lambda_{N} \bar{\tau}\right)$. For large $\bar{\tau}$, relations (257) and (258) are similar to (227) and (226), respectively. To show this, we use the relation $\overline{\mathbf{V}}_{-}=\widehat{\mathbf{V}}_{-} \mathbb{S}$, where $\mathbb{S}$ is a diagonal matrix of plus and minus ones, set $\widehat{\mathbf{V}}_{-}^{-T}=\left[\widetilde{\mathbf{v}}_{1}, \ldots, \widetilde{\mathbf{v}}_{N}\right]$, approximate $\Gamma \approx \operatorname{diag}\left[0, \ldots, 0, \exp \left(-\lambda_{N} \bar{\tau}\right)\right]$, and neglect the second term in the expression of $\widehat{\mathbf{T}}_{1}$ as well as the third term in the expression of $\widehat{\mathbf{R}}_{1}$. We obtain

$$
\begin{aligned}
& \widehat{\mathbf{T}}_{1}=s_{N} \mathrm{e}^{-\lambda_{N} \bar{\tau}} \widetilde{\mathbf{v}}_{N} \widetilde{\mathbf{v}}_{N}^{T}, \\
& \widehat{\mathbf{R}}_{1}=\widehat{\mathbf{R}}_{\infty}-s_{N} a_{N N} \mathrm{e}^{-2 \lambda_{N} \bar{\tau}} \widetilde{\mathbf{v}}_{N} \widetilde{\mathbf{v}}_{N}^{T},
\end{aligned}
$$

where $s_{N}=[\mathbb{S}]_{N N}, a_{N N}=[\mathbb{A}]_{N N}$, and $\widehat{\mathbf{R}}_{\infty}=\widehat{\mathbf{V}}_{+} \widehat{\mathbf{V}}_{-}^{-1}$. Comparing (259) and (260) with (227) and (226) under the assumptions $\widehat{\mathrm{r}} \approx \mathrm{k} \operatorname{lexp}(-2 \mathrm{k} \bar{\tau})$ and $\widehat{\mathrm{t}} \approx \operatorname{kexp}(-\mathrm{k} \bar{\tau})$ for $\bar{\tau} \gg 1$ (the Taylor expansions in $x=\exp (-\mathrm{k} \bar{\tau})$ of (229) and (230) for small $x$ ), we see that for the azimuthal mode $m=0$, we must have that $s_{N}=1, \lambda_{N}=\mathrm{k}, \widetilde{\mathbf{v}}_{N}=\sqrt{\mathbf{k}} \widehat{\mathbf{k}}_{N}$, and $a_{N N}=1$. Thus, by using Waterman's technique we were able to derive new representations for $\widehat{\mathbf{R}}_{\infty}, \widehat{\mathbf{k}}_{N}$ and 1 .

In Figure 4 we show the relative errors in the discrete approximations to the reflection and transmission functions computed by using (234)-(237) with $K=5, K=1$, and $K=0$ (asymptotic model), and the first-order Waterman's approximation (257)-(258). Obviously, Waterman's approximation yields the smallest errors in the transmission matrix, and sufficiently small errors in the reflection matrix.

\section{Matrix Riccati equations}

Bellman et al. [20] formulated the radiative transfer problem as an initial value problem via a pair of nonlinear matrix differential equations (matrix Riccati equations) which describe the reflection and transmission matrices in a planeparallel geometry. The derivation of Bellman et al. [20] is based on the invariant imbedding technique, and for this reason, the invariant imbedding is usually a synonym for the matrix Riccati equations method.

Flatau and Stephens [22], showed that for an inhomogeneous atmosphere, the matrix Riccati equations can be derived by means of an approach based on the propagator operator, that is, by an approach which is close related to the matrix exponential method. The main problem which arises in the case of an inhomogeneous atmosphere is that in view of

$\frac{\mathrm{d}}{\mathrm{d} \tau}\left(\mathrm{e}^{\mathbf{X}(\tau)}\right) \neq \mathbf{X}^{\prime}(\tau) \mathrm{e}^{\mathbf{X}(\tau)}$

the homogeneous solution of radiative transfer equation

$\frac{\mathrm{d} \widehat{\mathbf{i}}_{\mathrm{h}}}{\mathrm{d} \tau}(\tau)=-\widehat{\mathbf{A}}(\tau) \widehat{\mathbf{i}}_{\mathrm{h}}(\tau)$,

with the initial condition $\widehat{\mathbf{i}}_{\mathrm{h}}(0)$ and a continuous matrix $\widehat{\mathbf{A}}(\tau)$ on $0 \leq \tau \leq \bar{\tau}$, cannot be expressed in terms of the matrix exponential, i.e.,

$\widehat{\mathbf{i}}_{\mathrm{h}}(\tau) \neq \mathrm{e}^{-\int_{0}^{\tau} \widehat{\mathbf{A}}\left(\tau^{\prime}\right) \mathrm{d} \tau^{\prime} \widehat{\mathbf{i}}_{\mathrm{h}}(0) .}$

This fact should not be discouraged because in the matrix Riccati equation method we need only a formal solution representation of (262). The formal solution is [22]

$\widehat{\mathbf{i}}_{\mathrm{h}}(\tau)=\mathbf{P}_{-}(\tau, 0) \widehat{\mathbf{i}}_{\mathrm{h}}(0)$,

where the downward propagator $\mathbf{P}_{-}(\tau, 0)$ is a generalization of the propagator $\mathbf{P}(\tau)=\exp (-\widehat{\mathbf{A}} \tau)$ in the case of an inhomogeneous atmosphere. Inserting (264) in (262), we find that the downward propagator solves the differential equation

$\frac{\mathrm{d} \mathbf{P}_{-}}{\mathrm{d} \tau}(\tau, 0)=-\widehat{\mathbf{A}}(\tau) \mathbf{P}_{-}(\tau, 0)$,

with the initial condition $\mathbf{P}_{-}(0,0)=\mathbf{I}_{2 N}$. The subscript means that the propagation of the solution occurs from the level 0 (second argument of $\mathbf{P}_{-}$) downward to the level $\tau$ (first argument of $\mathbf{P}_{-}$). The propagation of the solution from the bottom to the top of the atmosphere is described by the upward propagator $\mathbf{P}_{+}(\tau, \bar{\tau})$ which is defined by $\hat{\mathbf{i}}_{\mathrm{h}}(\tau)=\mathbf{P}_{+}(\tau, \bar{\tau}) \hat{\mathbf{i}}_{\mathrm{h}}(\bar{\tau})$, and solve (265) with the initial condition $\mathbf{P}_{+}(\bar{\tau}, \bar{\tau})=\mathbf{I}_{2 N}$.

In an inhomogeneous atmosphere, the radiation coming from above will be reflected and transmitted differently 
than the radiation incident from below, so that in the downward scheme, the interaction principle for an inhomogeneous layer extending from the level 0 downward to the level $\tau$, reads as

$$
\left[\begin{array}{c}
\widehat{\mathbf{i}}_{\mathrm{h}}^{+}(0) \\
\widehat{\mathbf{i}}_{\mathrm{h}}^{-}(\tau)
\end{array}\right]=\left[\begin{array}{cc}
\widehat{\mathbf{R}}_{-}(\tau, 0) & \widehat{\mathbf{T}}_{+}(\tau, 0) \\
\widehat{\mathbf{T}}_{-}(\tau, 0) & \widehat{\mathbf{R}}_{+}(\tau, 0)
\end{array}\right]\left[\begin{array}{c}
\widehat{\mathbf{i}}_{\mathrm{h}}^{-}(0) \\
\widehat{\mathbf{i}}_{\mathrm{h}}^{+}(\tau)
\end{array}\right] .
$$

The notation $\widehat{\mathbf{R}}_{-}\left(\tau, \tau_{0}\right)$ stands for the reflection matrix of a layer of optical thickness $\tau$ illuminated from above, and whose top is placed at $\tau_{0}$. Note that in the upward scheme, the interaction principle equation relates the outcoming radiances $\widehat{\mathbf{i}}_{\mathrm{h}}^{+}(\tau)$ and $\widehat{\mathbf{i}}_{\mathrm{h}}^{-}(\bar{\tau})$ to the incoming radiances $\widehat{\mathbf{i}}_{\mathrm{h}}^{-}(\tau)$ and $\widehat{\mathbf{i}}_{\mathrm{h}}^{+}(\bar{\tau})$ through the reflection and transmission matrices $\widehat{\mathbf{R}}_{ \pm}(\bar{\tau}-$ $\tau, \tau)$ and $\widehat{\mathbf{T}}_{ \pm}(\bar{\tau}-\tau, \tau)$, respectively. From (264) and (266), we find

$$
\begin{aligned}
& \mathbf{P}_{-}(\tau, 0) \\
& =\left[\begin{array}{cc}
\widehat{\mathbf{T}}_{+}^{-1}(\tau, 0) & -\widehat{\mathbf{T}}_{+}^{-1}(\tau, 0) \widehat{\mathbf{R}}_{-}(\tau, 0) \\
\widehat{\mathbf{R}}_{+}(\tau, 0) \widehat{\mathbf{T}}_{+}^{-1}(\tau, 0) & \widehat{\mathbf{T}}_{-}(\tau, 0)-\widehat{\mathbf{R}}_{+}(\tau, 0) \widehat{\mathbf{T}}_{+}^{-1}(\tau, 0) \widehat{\mathbf{R}}_{-}(\tau, 0)
\end{array}\right],
\end{aligned}
$$

which is an extension of the matrix exponential representation (102) to the inhomogeneous case.

Taking the derivative of (267) and accounting of (265), that is,

$$
\begin{aligned}
& \frac{\mathrm{d}}{\mathrm{d} \tau}\left[\begin{array}{cc}
\widehat{\mathbf{T}}_{+}^{-1} & -\widehat{\mathbf{T}}_{+}^{-1} \widehat{\mathbf{R}}_{-} \\
\widehat{\mathbf{R}}_{+} \widehat{\mathbf{T}}_{+}^{-1} & \widehat{\mathbf{T}}_{-}-\widehat{\mathbf{R}}_{+} \widehat{\mathbf{T}}_{+}^{-1} \widehat{\mathbf{R}}_{-}
\end{array}\right] \\
& =-\left[\begin{array}{cc}
\widehat{\mathbf{A}}_{11} & \widehat{\mathbf{A}}_{12} \\
-\widehat{\mathbf{A}}_{12} & -\widehat{\mathbf{A}}_{11}
\end{array}\right]\left[\begin{array}{cc}
\widehat{\mathbf{T}}_{+}^{-1} & -\widehat{\mathbf{T}}_{+}^{-1} \widehat{\mathbf{R}}_{-} \\
\widehat{\mathbf{R}}_{+} \widehat{\mathbf{T}}_{+}^{-1} & \widehat{\mathbf{T}}_{-}-\widehat{\mathbf{R}}_{+} \widehat{\mathbf{T}}_{+}^{-1} \widehat{\mathbf{R}}_{-}
\end{array}\right],
\end{aligned}
$$

gives

$\frac{\mathrm{d}}{\mathrm{d} \tau} \widehat{\mathbf{R}}_{+}=\widehat{\mathbf{A}}_{12}+\widehat{\mathbf{A}}_{11} \widehat{\mathbf{R}}_{+}+\widehat{\mathbf{R}}_{+} \widehat{\mathbf{A}}_{11}+\widehat{\mathbf{R}}_{+} \widehat{\mathbf{A}}_{12} \widehat{\mathbf{R}}_{+}$,

$\frac{\mathrm{d}}{\mathrm{d} \tau} \widehat{\mathbf{T}}_{-}=\widehat{\mathbf{A}}_{11} \widehat{\mathbf{T}}_{-}+\widehat{\mathbf{R}}_{+} \widehat{\mathbf{A}}_{12} \widehat{\mathbf{T}}_{-}$,

and

$\frac{\mathrm{d}}{\mathrm{d} \tau} \widehat{\mathbf{R}}_{-}=\widehat{\mathbf{T}}_{+} \widehat{\mathbf{A}}^{12} \widehat{\mathbf{T}}_{-}$,

$\frac{\mathrm{d}}{\mathrm{d} \tau} \widehat{\mathbf{T}}_{+}=\widehat{\mathbf{T}}_{+} \widehat{\mathbf{A}}^{11}+\widehat{\mathbf{T}}_{+} \widehat{\mathbf{A}}^{12} \widehat{\mathbf{R}}_{+}$,

From $\mathbf{P}_{-}(\mathbf{0}, \mathbf{0})=\mathbf{I}_{2 N}$ and (267), we find the initial conditions

$$
\begin{aligned}
& \widehat{\mathbf{R}}_{+}(0,0)=\widehat{\mathbf{R}}_{-}(0,0)=\mathbf{0}, \\
& \widehat{\mathbf{T}}_{-}(0,0)=\widehat{\mathbf{T}}_{+}(0,0)=\mathbf{I}_{N} .
\end{aligned}
$$

By inspection of (269)-(272), it is apparent that equation (269) can be solved independently for $\widehat{\mathbf{R}}_{+}$, equation (270) as well as equation (272), must be solved along with (269) to find $\widehat{\mathbf{T}}_{-}$ and $\widehat{\mathbf{T}}_{+}$, respectively, while equation (271) must be solved together with (269), (270) and (272) to find $\widehat{\mathbf{R}}_{-}$. In summary, for an inhomogeneous atmosphere we started with the interaction principle equation (266), used relation (267) connecting the downward propagator and the extinction matrix, and then, by making use on the differential equation solved by the downward propagator (265), derived the matrix Riccati equations (269)-(272). Essentially, we replaced the problem of computing the downward propagator by the problem of computing the reflection and transmission matrices.

\subsection{Reflection and transmission matrices of a homogeneous layer}

For a homogeneous layer, the propagator $\mathbf{P}_{-}(\tau, 0)=\mathbf{P}(\tau)=$ $\exp (-\widehat{\mathbf{A}} \tau)$ solves the differential equation

$\frac{\mathrm{d}\left(\mathrm{e}^{-\widehat{\mathbf{A}} \tau}\right)}{\mathrm{d} \tau}=-\widehat{\mathbf{A}} \mathrm{e}^{-\widehat{\mathbf{A}} \tau}$,

and (269)-(272) simplify to

$\frac{\mathrm{d}}{\mathrm{d} \tau} \widehat{\mathbf{R}}=\widehat{\mathbf{A}}_{12}+\widehat{\mathbf{A}}_{11} \widehat{\mathbf{R}}+\widehat{\mathbf{R}} \widehat{\mathbf{A}}_{11}+\widehat{\mathbf{R}} \widehat{\mathbf{A}}_{12} \widehat{\mathbf{R}}$,

$\frac{\mathrm{d}}{\mathrm{d} \tau} \widehat{\mathbf{T}}=\widehat{\mathbf{A}}_{11} \widehat{\mathbf{T}}+\widehat{\mathbf{R}} \widehat{\mathbf{A}}_{12} \widehat{\mathbf{T}}$,

and

$\frac{\mathrm{d}}{\mathrm{d} \tau} \widehat{\mathbf{R}}=\widehat{\mathbf{T}} \widehat{\mathbf{A}}_{12} \widehat{\mathbf{T}}$,

$\frac{\mathrm{d}}{\mathrm{d} \tau} \widehat{\mathbf{T}}=\widehat{\mathbf{T}} \widehat{\mathbf{A}}_{11}+\widehat{\mathbf{T}} \widehat{\mathbf{A}}_{12} \widehat{\mathbf{R}}$.

where $\widehat{\mathbf{R}}_{+}(\tau, 0)=\widehat{\mathbf{R}}_{-}(\tau, 0)=\widehat{\mathbf{R}}(\tau)$ and $\widehat{\mathbf{T}}_{+}(\tau, 0)=\widehat{\mathbf{T}}_{-}(\tau, 0)=\widehat{\mathbf{T}}(\tau)$ . From (276)-(279), we see that the following identities must hold

$$
\widehat{\mathbf{T}} \widehat{\mathbf{A}}_{12} \widehat{\mathbf{T}}=\widehat{\mathbf{A}}_{12}+\widehat{\mathbf{A}}_{11} \widehat{\mathbf{R}}+\widehat{\mathbf{R}} \widehat{\mathbf{A}}_{11}+\widehat{\mathbf{R}} \widehat{\mathbf{A}}_{12} \widehat{\mathbf{R}},
$$

$\widehat{\mathbf{T}} \widehat{\mathbf{A}}_{11}+\widehat{\mathbf{T}} \widehat{\mathbf{A}}_{12} \widehat{\mathbf{R}}=\widehat{\mathbf{A}}_{11} \widehat{\mathbf{T}}+\widehat{\mathbf{R}} \widehat{\mathbf{A}}_{12} \widehat{\mathbf{T}}$.

It should perhaps be pointed out that (276)-(279) may follow directly from (102) and (275).

The solutions of the matrix Riccati equations for a homogeneous layer must be identical with the representations of the reflection and transmission matrices derived by the matrix exponential method of Section 5. Let us prove this result. The initial value problem for the reflection matrix (276) and (273) is equivalent with the following $2 n$th order linear initial value problem

$$
\begin{aligned}
\frac{\mathrm{d}}{\mathrm{d} \tau}\left[\begin{array}{c}
\mathscr{P}(\tau) \\
\mathscr{Q}(\tau)
\end{array}\right] & =-\left[\begin{array}{cc}
\widehat{\mathbf{A}}_{11} & \widehat{\mathbf{A}}_{12} \\
-\widehat{\mathbf{A}}_{12} & -\widehat{\mathbf{A}}_{11}
\end{array}\right]\left[\begin{array}{c}
\mathscr{P}(\tau) \\
\mathscr{Q}(\tau)
\end{array}\right], \\
{\left[\begin{array}{c}
\mathscr{P}(0) \\
\mathscr{Q}(0)
\end{array}\right] } & =\left[\begin{array}{c}
\mathbf{I}_{N} \\
0
\end{array}\right],
\end{aligned}
$$

where

$\widehat{\mathbf{R}}(\tau)=\mathscr{Q}(\tau) \mathscr{P}^{-1}(\tau)$.

Making the change of variable

$$
\left[\begin{array}{c}
\widehat{\mathscr{P}}(\tau) \\
\widehat{\mathscr{Q}}(\tau)
\end{array}\right]=\left[\begin{array}{cc}
-\overline{\mathbf{V}}_{+}^{T} & \overline{\mathbf{V}}_{-}^{T} \\
\overline{\mathbf{V}}_{-}^{T} & -\overline{\mathbf{V}}_{+}^{T}
\end{array}\right]\left[\begin{array}{c}
\mathscr{P}(\tau) \\
\mathscr{Q}(\tau)
\end{array}\right],
$$


the linear differential equation (282) becomes

$\frac{\mathrm{d}}{\mathrm{d} \tau}\left[\begin{array}{c}\widehat{\mathscr{P}}(\tau) \\ \widehat{\mathscr{Q}}(\tau)\end{array}\right]=-\left[\begin{array}{cc}\boldsymbol{\Lambda} & \mathbf{0} \\ \mathbf{0} & -\boldsymbol{\Lambda}\end{array}\right]\left[\begin{array}{c}\widehat{\mathscr{P}}(\tau) \\ \widehat{\mathscr{Q}}(\tau)\end{array}\right]$,

with the initial condition

$$
\left[\begin{array}{c}
\widehat{\mathscr{P}}(0) \\
\widehat{\mathscr{Q}}(0)
\end{array}\right]=\left[\begin{array}{c}
-\overline{\mathbf{V}}_{+}^{T} \\
\overline{\mathbf{V}}_{-}^{T}
\end{array}\right] .
$$

In terms of $\widehat{\mathscr{P}}$ and $\widehat{\mathscr{Q}}$, the expression of the reflection matrix reads as

$\widehat{\mathbf{R}}(\tau)=\left[\widehat{\mathbf{V}}_{-} \widehat{\mathscr{P}}(\tau) \widehat{\mathscr{Q}}^{-1}(\tau)+\widehat{\mathbf{V}}_{+}\right]\left[\widehat{\mathbf{V}}_{+} \widehat{\mathscr{P}}(\tau) \widehat{\mathscr{Q}}^{-1}(\tau)+\widehat{\mathbf{V}}_{-}\right]^{-1}$

The solution of the differential equation (285) is given by $\widehat{\mathscr{P}}(\tau)=\left[\mathrm{e}^{-\lambda_{k} \tau}\right] \widehat{\mathscr{P}}(0)$ and $\widehat{\mathscr{Q}}(\tau)=\left[\mathrm{e}^{\lambda_{k} \tau}\right] \widehat{\mathscr{Q}}(0)$, so that the matrix product $\widehat{\mathscr{P}}(\tau) \widehat{\mathscr{Q}}^{-1}(\tau)$ in (287) can be expressed as

$\widehat{\mathscr{P}}(\tau) \widehat{\mathscr{Q}}^{-1}(\tau)=\boldsymbol{\Gamma}(\tau) \widehat{\mathscr{P}}(0) \widehat{\mathscr{Q}}^{-1}(0) \boldsymbol{\Gamma}(\tau)=-\boldsymbol{\Gamma}(\tau) \overline{\mathbf{V}}_{+}^{T} \overline{\mathbf{V}}_{-}^{-T} \boldsymbol{\Gamma}(\tau)$

Then, employing the identity (cf. (48)) $\overline{\mathbf{V}}_{+}^{T} \overline{\mathbf{V}}_{-}^{-T}=\widehat{\mathbf{V}}_{-}^{-1} \widehat{\mathbf{V}}_{+}$, in (288), and inserting the resulting expression in (287), we obtain

$\widehat{\mathbf{R}}(\tau)=\left[\widehat{\mathbf{V}}_{+}-\widehat{\mathbf{V}}_{-} \boldsymbol{\Gamma}(\tau) \widehat{\mathbf{V}}_{-}^{-1} \widehat{\mathbf{V}}_{+} \boldsymbol{\Gamma}(\tau)\right]\left[\widehat{\mathbf{V}}_{-}-\widehat{\mathbf{V}}_{+} \boldsymbol{\Gamma}(\tau) \widehat{\mathbf{V}}_{-}^{-1} \widehat{\mathbf{V}}_{+} \boldsymbol{\Gamma}(\tau)\right]^{-1}$,

which is exactly the reflection matrix representation (94) for $\tau=\bar{\tau}$.

\subsection{Approximations based on matrix Riccati equations}

A benefit of the matrix Riccati equation formalism is that for small values of the optical thickness and/or single scattering albedo, several approximation methods can be derived. These methods are summarized below.

\subsubsection{Successive orders of scattering}

In the matrix Riccati equations for a homogeneous layer

$\frac{\mathrm{d}}{\mathrm{d} \tau} \widehat{\mathbf{R}}=\widehat{\mathbf{A}}_{12}+\widehat{\mathbf{A}}_{11} \widehat{\mathbf{R}}+\widehat{\mathbf{R}} \widehat{\mathbf{A}}_{11}+\widehat{\mathbf{R}} \widehat{\mathbf{A}}_{12} \widehat{\mathbf{R}}$,

$\frac{\mathrm{d}}{\mathrm{d} \tau} \widehat{\mathbf{T}}=\widehat{\mathbf{A}}_{11} \widehat{\mathbf{T}}+\widehat{\mathbf{R}} \widehat{\mathbf{A}}_{12} \widehat{\mathbf{T}}$,

we separate the attenuation terms from the multiplescattering terms, by defining the matrix $\widehat{\mathbf{A}}_{11}^{0}$ through the relation

$\widehat{\mathbf{A}}_{11}^{0}=\widehat{\mathbf{A}}_{11}+\mathbf{M}$

and indicate explicitly the dependency of the matrices $\widehat{\mathbf{A}}_{11}^{0}$ and $\widehat{\mathbf{A}}_{12}$ on the single scattering albedo $\omega$ by writing

$\widehat{\mathbf{A}}_{11}^{0}=\omega \widetilde{\mathbf{A}}_{11}^{0}$ and $\widehat{\mathbf{A}}_{12}=\omega \widetilde{\mathbf{A}}_{12}$.
The resulting matrix Riccati equations, namely

$$
\begin{aligned}
& \frac{\mathrm{d}}{\mathrm{d} \tau} \widehat{\mathbf{R}}+\mathbf{M} \widehat{\mathbf{R}}+\widehat{\mathbf{R}} \mathbf{M}=\omega \widetilde{\mathbf{A}}_{12}+\omega \widetilde{\mathbf{A}}_{11}^{0} \widehat{\mathbf{R}}+\omega \widehat{\mathbf{R}} \widetilde{\mathbf{A}}_{11}^{0}+\omega \widehat{\mathbf{R}} \widetilde{\mathbf{A}}_{12} \widehat{\mathbf{R}}, \\
& \frac{\mathrm{d}}{\mathrm{d} \tau} \widehat{\mathbf{T}}+\mathbf{M} \widehat{\mathbf{T}}=\omega \widetilde{\mathbf{A}}_{11}^{0} \widehat{\mathbf{T}}+\omega \widehat{\mathbf{R}} \widetilde{\mathbf{A}} 12 \widehat{\mathbf{T}},
\end{aligned}
$$

endowed with the initial conditions $\widehat{\mathbf{R}}(0)=0$ and $\widehat{\mathbf{T}}(0)=\mathbf{I}$, are usually referred to as the discrete invariant imbedding equations. As pointed out by Waterman, the "Padé and Taylor series expansions are fundamentally different from those involving successive orders of scattering; in the later, $\omega$ is employed as the expansion parameter". Along this line, we assume the $n$ th-order approximations

$\widehat{\mathbf{R}}_{n}(\tau, \omega)=\sum_{k=0}^{n} \omega^{k} \widehat{\mathbf{H}}_{k}(\tau)$,

$\widehat{\mathbf{T}}_{n}(\tau, \omega)=\sum_{k=0}^{n} \omega^{k} \widehat{\mathbf{G}}_{k}(\tau)$.

Inserting (296) and (297) into (294) and (295) and equating the coefficients of $\omega^{n}$, yields the iterative schemes

$\frac{\mathrm{d}}{\mathrm{d} \tau} \widehat{\mathbf{H}}_{0}(\tau)+\mathbf{M} \widehat{\mathbf{H}}_{0}(\tau)+\widehat{\mathbf{H}}_{0}(\tau) \mathbf{M}=0, \widehat{\mathbf{H}}_{0}(0)=0$,

$\frac{\mathrm{d}}{\mathrm{d} \tau} \widehat{\mathbf{H}}_{n}(\tau)+\mathbf{M} \widehat{\mathbf{H}}_{n}(\tau)+\widehat{\mathbf{H}}_{n}(\tau) \mathbf{M}=\delta_{n 1} \widetilde{\mathbf{A}}_{12}+\widetilde{\mathbf{A}}_{11}^{0} \widehat{\mathbf{H}}_{n-1}(\tau)$

$+\widehat{\mathbf{H}}_{n-1}(\tau) \widetilde{\mathbf{A}}_{11}^{0}+\sum_{k=0}^{n-1} \widehat{\mathbf{H}}_{k}(\tau) \widetilde{\mathbf{A}}_{12} \widehat{\mathbf{H}}_{n-k-1}(\tau), \widehat{\mathbf{H}}_{n}(0)=0, n \geq 1$,

and

$\frac{\mathrm{d}}{\mathrm{d} \tau} \widehat{\mathbf{G}}_{0}(\tau)+\mathbf{M} \widehat{\mathbf{G}}_{0}(\tau)=0 \widehat{\mathbf{G}}_{0}(0)=\mathbf{I}$,

$\frac{\mathrm{d}}{\mathrm{d} \tau} \widehat{\mathbf{G}}_{n}(\tau)+\mathbf{M} \widehat{\mathbf{G}}_{n}(\tau)=\widetilde{\mathbf{A}}_{11}^{0} \widehat{\mathbf{G}}_{n-1}(\tau)$

$+\sum_{k=0}^{n-1} \widehat{\mathbf{H}}_{k}(\tau) \widetilde{\mathbf{A}}_{12} \widehat{\mathbf{G}}_{n-k-1}(\tau), \widehat{\mathbf{G}}_{n}(0)=0, n \geq 1$.

For $n \geq 1$, the solutions of the initial value problems (299) and (301) are

$$
\begin{aligned}
{\left[\widehat{\mathbf{H}}_{n}(\tau)\right]_{k l} } & =\int_{0}^{\tau} \mathrm{e}^{-\left(\frac{1}{\mu_{k}}+\frac{1}{\mu_{l}}\right)\left(\tau-\tau^{\prime}\right)}\left[\mathbb{H}_{n}\left(\tau^{\prime}\right)\right]_{k l} \mathrm{~d} \tau^{\prime}, \\
\mathbb{H}_{n}(\tau) & =\delta_{n 1} \widetilde{\mathbf{A}}_{12}+\widetilde{\mathbf{A}}_{11}^{0} \widehat{\mathbf{H}}_{n-1}(\tau)+\widehat{\mathbf{H}}_{n-1}(\tau) \widetilde{\mathbf{A}}_{11}^{0} \\
& +\sum_{k=0}^{n-1} \widehat{\mathbf{H}}_{k}(\tau) \widetilde{\mathbf{A}}_{12} \widehat{\mathbf{H}}_{n-k-1}(\tau),
\end{aligned}
$$

and

$$
\begin{aligned}
{\left[\widehat{\mathbf{G}}_{n}(\tau)\right]_{k l} } & =\int_{0}^{\tau} \mathrm{e}^{-\frac{1}{\mu_{k}}\left(\tau-\tau^{\prime}\right)}\left[\mathbb{G}_{n}\left(\tau^{\prime}\right)\right]_{k l} \mathrm{~d} \tau^{\prime}, \\
\mathbb{G}_{n}(\tau) & =\widetilde{\mathbf{A}}_{11}^{0} \widehat{\mathbf{G}}_{n-1}(\tau)+\sum_{k=0}^{n-1} \widehat{\mathbf{H}}_{k}(\tau) \widetilde{\mathbf{A}}_{12} \widehat{\mathbf{G}}_{n-k-1}(\tau),
\end{aligned}
$$

respectively, with $\widehat{\mathbf{H}}_{0}(\tau)=0$ and $\left[\widehat{\mathbf{G}}_{0}(\tau)\right]_{k l}=\delta_{k l} \exp \left(-\tau / \mu_{k}\right)$. Accordingly, the zeroth- and first-order scattering solutions are

$\widehat{\mathbf{R}}_{0}(\bar{\tau})=0,\left[\widehat{\mathbf{T}}_{0}(\bar{\tau})\right]_{k l}=\delta_{k l} \mathrm{e}^{-\frac{1}{\mu_{k}} \bar{\tau}}$, 
and

$$
\begin{aligned}
& {\left[\widehat{\mathbf{R}}_{1}(\bar{\tau})\right]_{k l}=\left(\frac{1}{\mu_{k}}+\frac{1}{\mu_{l}}\right)^{-1}\left[1-\mathrm{e}^{-\left(\frac{1}{\mu_{k}}+\frac{1}{\mu_{l}}\right) \bar{\tau}}\right]\left[\widehat{\mathbf{A}}_{12}\right]_{k l},} \\
& {\left[\widehat{\mathbf{T}}_{1}(\bar{\tau})\right]_{k l}=\delta_{k l} \mathrm{e}^{-\frac{1}{\mu_{k}} \bar{\tau}}+\left(\frac{1}{\mu_{k}}-\frac{1}{\mu_{l}}\right)^{-1}\left(\mathrm{e}^{-\frac{1}{\mu_{l}} \bar{\tau}}-\mathrm{e}^{-\frac{1}{\mu_{k}} \bar{\tau}}\right)\left[\widehat{\mathbf{A}}_{11}^{0}\right]_{k l},}
\end{aligned}
$$

respectively. Analytical formulas for the first three orders of scattering, which for most applications are sufficient, have been derived by Kawabata and Ueno [48]. However, the resulting expressions are too complex, so that even in the case $n=2$, it is more efficient to use the Gaussian quadrature method to compute $\widehat{\mathbf{H}}_{2}$ and $\widehat{\mathbf{G}}_{2}$ by means of (302) and (304), respectively. It should be pointed out that the errors in $\widehat{\mathbf{R}}_{2}$ are considerably smaller than the errors in $\widehat{\mathbf{T}}_{2}$. For example, we found that for $\bar{\tau}=20$ and $\omega=0.2$, the relative errors in the second-order reflection and transmission functions are $8.14 \cdot 10^{-3}$ and $6.07 \cdot 10^{-2}$, respectively. This can be explained by the fact that in the case of strong absorption, the refection is determined by the (relatively thin) skin layer, while transmission is determined by the whole layer and requires more scattering.

Alternative recurrence relations for $\widehat{\mathbf{H}}_{n}(\tau)$ and $\widehat{\mathbf{G}}_{n}(\tau)$, which do not involve an integration over the optical depth, can be obtained by considering the identities (280) and (281) which, by means of (292) and (293), can be written as

$$
\begin{aligned}
\mathbf{M} \widehat{\mathbf{R}}+\widehat{\mathbf{R}} \mathbf{M} & =\omega \widetilde{\mathbf{A}}_{12}+\omega \widetilde{\mathbf{A}}_{11}^{0} \widehat{\mathbf{R}}+\omega \widehat{\mathbf{R}} \widetilde{\mathbf{A}}_{11}^{0} \\
& +\omega \widehat{\mathbf{R}} \widetilde{\mathbf{A}}_{12} \widehat{\mathbf{R}}-\omega \widehat{\mathbf{T}} \widetilde{\mathbf{A}}_{12} \widehat{\mathbf{T}} \\
\mathbf{M} \widehat{\mathbf{T}}-\widehat{\mathbf{T}} \mathbf{M} & =\omega \widetilde{\mathbf{A}}_{11}^{0} \widehat{\mathbf{T}}-\omega \widehat{\mathbf{T}} \widetilde{\mathbf{A}}_{11}^{0}+\omega \widehat{\mathbf{R}} \widetilde{\mathbf{A}}_{12} \widehat{\mathbf{T}}-\omega \widehat{\mathbf{T}} \widetilde{\mathbf{A}}_{12} \widehat{\mathbf{R}} .
\end{aligned}
$$

Substituting (296) and (297) into (309) and (310), and equating the coefficients of $\omega^{n}$ gives for $n \geq 1$,

$$
\begin{aligned}
& \mathbf{M} \widehat{\mathbf{H}}_{n}(\tau)+\widehat{\mathbf{H}}_{n}(\tau) \mathbf{M}=\delta_{n 1} \widetilde{\mathbf{A}}_{12}+\widetilde{\mathbf{A}}_{11}^{0} \widehat{\mathbf{H}}_{n-1}(\tau)+\widehat{\mathbf{H}}_{n-1}(\tau) \widetilde{\mathbf{A}}_{11}^{0} \\
& +\sum_{k=0}^{n-1} \widehat{\mathbf{H}}_{k}(\tau) \widetilde{\mathbf{A}}_{12} \widehat{\mathbf{H}}_{n-k-1}(\tau)-\sum_{k=0}^{n-1} \widehat{\mathbf{G}}_{k}(\tau) \widetilde{\mathbf{A}}_{12} \widehat{\mathbf{G}}_{n-k-1}(\tau)
\end{aligned}
$$

and

$$
\begin{aligned}
& \mathbf{M} \widehat{\mathbf{G}}_{n}(\tau)-\widehat{\mathbf{G}}_{n}(\tau) \mathbf{M}=\widetilde{\mathbf{A}}_{11}^{0} \widehat{\mathbf{G}}_{n-1}(\tau)+\widehat{\mathbf{G}}_{n-1}(\tau) \widetilde{\mathbf{A}}_{11}^{0} \\
& +\sum_{k=0}^{n-1} \widehat{\mathbf{H}}_{k}(\tau) \widetilde{\mathbf{A}}_{12} \widehat{\mathbf{G}}_{n-k-1}(\tau)-\sum_{k=0}^{n-1} \widehat{\mathbf{G}}_{k}(\tau) \widetilde{\mathbf{A}}_{12} \widehat{\mathbf{H}}_{n-k-1}(\tau),
\end{aligned}
$$

with $\widehat{\mathbf{H}}_{0}(\tau)=0$ and $\left[\widehat{\mathbf{G}}_{0}(\tau)\right]_{k l}=\delta_{k l} \exp \left(-\tau / \mu_{k}\right)$. Equations (311) and (312) have been derived by Hansen and Travis [3] using the invariance principle. Note that (312) is indeterminate for $\mu_{k}=\mu_{l}$, and that approximate results for that special case can be obtained by interpolation.

\subsubsection{Iterative approximation}

For thin layers, the Taylor series approximations to the transmission and reflection matrices (175) and (176), can be rediscover by using the matrix Riccati equation method. In this regard, we mention that Chang and Wu [49] solved (290) and (291) with the initial conditions $\widehat{\mathbf{R}}(0)=0$ and $\widehat{\mathbf{T}}(0)=\mathbf{I}$ by means of the iterative scheme

$$
\begin{aligned}
\widehat{\mathbf{R}}_{1}(\tau) & =\int_{0}^{\tau} \widehat{\mathbf{A}}_{12} \mathrm{~d} \tau^{\prime}, \\
\widehat{\mathbf{R}}_{n}(\tau) & =\int_{0}^{\tau}\left[\widehat{\mathbf{A}}_{12}+\widehat{\mathbf{A}}_{11} \widehat{\mathbf{R}}_{n-1}\left(\tau^{\prime}\right)\right. \\
& \left.+\widehat{\mathbf{R}}_{n-1}\left(\tau^{\prime}\right) \widehat{\mathbf{A}}_{11}+\widehat{\mathbf{R}}_{n-1}\left(\tau^{\prime}\right) \widehat{\mathbf{A}}_{12} \widehat{\mathbf{R}}_{n-1}\left(\tau^{\prime}\right)\right] \mathrm{d} \tau^{\prime}, n \geq 2,
\end{aligned}
$$

and

$\widehat{\mathbf{T}}_{1}(\tau)=\mathbf{I}+\int_{0}^{\tau} \widehat{\mathbf{A}}_{11} \mathrm{~d} \tau^{\prime}$

$\widehat{\mathbf{T}}_{n}(\tau)=\mathbf{I}+\int_{0}^{\tau}\left[\widehat{\mathbf{A}}_{11} \widehat{\mathbf{T}}_{n-1}\left(\tau^{\prime}\right)+\widehat{\mathbf{R}}_{n-1}\left(\tau^{\prime}\right) \widehat{\mathbf{A}}_{12} \widehat{\mathbf{T}}_{n-1}\left(\tau^{\prime}\right)\right] \mathrm{d} \tau^{\prime}, n \geq 2$.

It is not hard to see that the first-order solutions $\widehat{\mathbf{T}}_{1}$ and $\widehat{\mathbf{R}}_{1}$ correspond to the infinitesimal generator initialization scheme of Grant and Hunt [39], while the second-order solutions $\widehat{\mathbf{T}}_{2}$ and $\widehat{\mathbf{R}}_{2}$ (excepting the terms in $\bar{\tau}^{3}$ ) correspond to the expanded diamond initialization scheme of Wiscombe [40]. To design a procedure for computing higher-order correction terms, we assume the finite Taylor expansions (175) and (176) for $\widehat{\mathbf{T}}_{n}$ and $\widehat{\mathbf{R}}_{n}$, respectively. Then, from (314) and (316) we obtain for $n \geq 2$,

$$
\begin{aligned}
\widehat{\mathbf{H}}_{n} & =\frac{1}{n}\left(\widehat{\mathbf{A}}_{11} \widehat{\mathbf{H}}_{n-1}+\widehat{\mathbf{H}}_{n-1} \widehat{\mathbf{A}}_{11}\right)+\frac{1}{n} \sum_{k=0}^{n-1} \widehat{\mathbf{H}}_{k} \widehat{\mathbf{A}}_{12} \widehat{\mathbf{H}}_{n-k-1}, \\
\widehat{\mathbf{G}}_{n} & =\frac{1}{n} \widehat{\mathbf{A}}_{11} \widehat{\mathbf{G}}_{n-1}+\frac{1}{n} \sum_{k=0}^{n-1} \widehat{\mathbf{H}}_{k} \widehat{\mathbf{A}}_{12} \widehat{\mathbf{G}}_{n-k-1},
\end{aligned}
$$

with $\widehat{\mathbf{H}}_{0}=0, \widehat{\mathbf{H}}_{1}=\widehat{\mathbf{A}}_{12}, \widehat{\mathbf{G}}_{0}=\mathbf{I}$, and $\widehat{\mathbf{G}}_{1}=\widehat{\mathbf{A}}_{11}$. Equations (317) and (318) are the counterparts of (180) and (178) in the matrix Riccati equation formalism.

For optical depths much less than unity, (290) and (291) can be also solved numerically by using an $n$ th-order RungeKutta scheme. As shown in [49], the fourth-order RungeKutta scheme has a higher computational cost than the fourth-order iterative method but is more accurate.

\section{Conclusions}

This paper provides a description of the matrix exponential formalism in radiative transfer. The solution of the initial value problem of the discrete radiative transfer is expressed in terms of the matrix exponential. Although, the matrix exponential solution is computationally unstable it is used as a starting point in deriving a computationally stable equation, the layer equation, which is the heart of the discrete ordinate method, the interaction principle equation, which is the heart of the matrix operator method, and finally, the matrix Riccati equations. Thus, the matrix exponential formalism gives the framework for the unification of the discrete ordinate method, the matrix operator method, and the matrix Riccati equations method. In our analysis, 
1. we provided an interpretation of the matrix exponential solution in terms of homogeneous and particular solutions, as well as in terms of total and direct radiance vectors,

2. we used the right- and left eigenvector technique of Waterman [21] to compute the matrix exponential, and derived a set of matrix identities for proving the mathematical equivalence between the different solution methods,

3. we established the layer equation of the discrete ordinate method with matrix exponential, compared this method with the classical discrete ordinate method, derived equivalent solution representations, and established the link between the matrix exponential solution and the Chandrasekhar's discrete ordinate solution,

4. we derived equivalent expressions for the reflection and transmission matrices by converting the layer equation and the solution representations of the matrix exponential method into the interaction principle equation,

5. for optically thin layers, we used the $n$ th-order Padé and Taylor series approximations to the matrix exponential to compute the reflection and transmission matrices, and to derive an $n$ th-order approximation to the layer equation,

6. for optically thick layers, we derived the asymptotic expressions of the reflection and transmission matrices by adapting the discrete ordinate approach of Nakajima and King [27] to our framework, obtained higherorder corrections of the reflection and transmission matrices for moderate values of the optical thickness, reconsidered Waterman's approximation by including an additional term in the expression of the reflection matrix, and computed parametrizations of the asymptotic functions and constants for a water-cloud model with a Gamma size distribution,

7. we reviewed the approach of Flatau and Stephens [22] for obtaining the matrix Riccati equations in the case of an inhomogeneous atmosphere, proved the equivalence between the matrix Riccati equations method and the eigendecomposition method in computing the reflection matrix of a homogeneous layer, and discussed the successive order of scattering approximation for small values of the single-scattering albedo, as well as, an $n$ thorder iterative approximation for small values of the optical thickness.

Some additional results are given in appendices. In Appendix 1 , we justify the choice of Gaussian quadrature in the discrete ordinate method. In Appendix 2 we review several eigendecomposition methods for computing the matrix exponential in a common framework. In this context it should be pointed out that the direct decomposition method is preferable for numerical implementations, while the square-root method is an important tool for theoretical studies, e.g., conservative scattering and asymptotic theory. In Appendix 3, we extend the analytical formulas for non-conservative scattering to conservative scattering, and prove that the system of char- acteristic solutions proposed by Nakajima and Tanaka [18] can be used for both non-conservative and conservative scattering.

The practical conclusion of our analysis is that the matrix exponential formalism enables the design of a radiative transfer code incorporating the discrete ordinate method, the matrix operator method and approximate models. For each homogeneous layer of a multi-layered atmosphere, a spectral decomposition of the layer matrix is performed, and then either the layer equation is derived and assembled into the system matrix of the entire atmosphere (discrete ordinate method), or the reflection and transmission matrices of the layer are computed and the adding formula is used to obtain the reflection matrix of the entire atmosphere (matrix operator method). Moreover, a combined model can be also designed.

To speed up the computational process, approximate models can be used. An efficient radiative transfer code should incorporate built-in routines that automatically work when the optical thickness of a homogeneous (sub)layer becomes too small or too large. On the other hand, the $n$ thorder Padé and Taylor series approximations and the $n$ thorder iterative approximation can be used for the initialization of the doubling method in radiative transfer, while the asymptotic form of the reflection function can be used in a cloud parameter retrieval algorithm. In the latter case, the computational process is organized as follows [50]: (I) replace the atmosphere below the cloud bottom by an equivalent Lambertian surface of albedo $A$, (II) compute the reflection function of a layer with an underlying Lambertian surface of albedo $A$ by means of the asymptotic theory, and (III) use the cloud reflection function as a bidirectional reflection function in a discrete ordinate model to compute the radiance field of the atmosphere above the cloud top.

\section{Appendix 1. Gauss quadrature}

In radiative transfer, the phase function $p$ is usually expressed through a finite series of normalized Legendre polynomials $P_{n}$, i.e.,

$p\left(\mu, \mu^{\prime}, \varphi-\varphi^{\prime}\right)=\sum_{n=0}^{N_{\max }} \sqrt{\frac{2 n+1}{2}} \chi_{n} P_{n}(\cos \Theta)$,

where $\cos \Theta=\mu \mu^{\prime}+\sqrt{1-\mu^{2}} \sqrt{1-\mu^{\prime 2}} \cos \left(\varphi-\varphi^{\prime}\right), \chi_{n}$ are the expansion coefficients, and $N_{\max }$ is the number of expansion coefficients. Making use on the addition theorem for the Legendre polynomials, we obtain an expansion in terms of spherical harmonics

$$
p\left(\mu, \mu^{\prime}, \varphi-\varphi^{\prime}\right)=\sum_{m=0}^{M_{\max }} \sum_{n=m}^{N_{\max }}\left(2-\delta_{m 0}\right) \chi_{n} P_{n}^{m}(\mu) P_{n}^{m}\left(\mu^{\prime}\right) \cos \left[m\left(\varphi-\varphi^{\prime}\right)\right],
$$

with $M_{\max } \leq N_{\max }$. We are now in the favorable situation that the kernel of the radiative transfer integral equation is 
expanded in an orthogonal and complete system of functions. Further on, we argue in connection with the spherical harmonics method. On the unit sphere, the diffuse radiance $I_{\mathrm{d}}\left(\tau, \mu^{\prime},-\mu_{0}, \varphi^{\prime}-\varphi_{0}\right)$ can be also expanded in terms of spherical harmonics, so that after integrating the multiple scattering term with respect to the azimuthal angle, we are led to an integral of the form $\int_{-1}^{1} P_{n}^{m}\left(\mu^{\prime}\right) P_{n^{\prime}}^{m}\left(\mu^{\prime}\right) \mathrm{d} \mu^{\prime}$, with $n, n^{\prime}=0, \ldots, N_{\max }$. For $n, n^{\prime} \leq N_{\max }, P_{n}^{m}\left(\mu^{\prime}\right) P_{n^{\prime}}^{n^{\prime}}\left(\mu^{\prime}\right)=P\left(\mu^{\prime}\right)$ is a polynomial of degree at most $2 N_{\max }$. This can be seen by making use on the explicit construction of the associated Legendre functions in terms of Jacobi polynomials. The integral then reduces to $\int_{-1}^{1} P\left(\mu^{\prime}\right) \mathrm{d} \mu^{\prime}$, and the task is to find an exact quadrature for this integral. If this is done, the mathematical equivalence between the spherical harmonics and the discrete ordinate method is established. In general, a Gauss quadrature using $N_{\mathrm{q}}$ nodes is an exact quadrature for polynomials of degree $2 N_{\mathrm{q}}-1$ or less [51]. In our case, this condition translates into $N_{\max }<N_{\mathrm{q}}-1 / 2$, and for the choice $N_{\mathrm{q}}=2 N$, it follows that $N_{\max }<2 N-1 / 2$. Thus, using $2 N$ Gaussian nodes and weights, we need $N_{\max }=2 N-1$ expansion terms in (319) and $M_{\max } \leq 2 N-1$ azimuthal modes. In [52] it is shown that

1. a Gauss quadrature guarantees that the phase function is correctly normalized, i.e., $\int_{0}^{2 \pi} \int_{-1}^{1} p\left(\mu, \mu^{\prime}, \varphi-\varphi^{\prime}\right) \mathrm{d} \mu^{\prime} \mathrm{d} \varphi^{\prime}=$ $4 \pi$, and so, that the energy is conserved in the computation,

2. a double Gauss quadrature, in which the Gaussian formula is applied separateley to the half ranges $(-1,0)$ and $(0,1)$, is preferable that a Gauss quadrature for the complete range $(-1,1)$.

It is a fact that Gauss quadrature has a factor-of-2 advantage in its efficiency as compared to equidistant quadrature methods. A method wich has almost the same performances and can be implemented effortlessly by the fast Fourier transform is the Clenshaw-Curtis scheme [53]. However, as it has been shown in [54], when the number of nodes $N_{\mathrm{q}}$ increases, the error of the Clenshaw-Curtis quadrature does not decay to zero evenly but in two distinct stages; for $N_{\mathrm{q}}$ smaller than a critical value, the error decreases by the rate $O\left(\rho^{-2 N_{\mathrm{q}}}\right)$, where $\rho>1$, and afterwards by the rate $O\left(\rho^{-N_{\mathrm{q}}}\right)$. This means that initially (for small $N_{\mathrm{q}}$ ), Clenshaw-Curtis quadrature converges about as fast as the Gauss quadrature. The outlook for a future work is to focus on the efficiency of the ClenshawCurtis quadrature in radiative transfer.

\section{Appendix 2. Spectral decomposition of the layer matrix}

Let us consider the layer matrix

$\widehat{\mathbf{A}}=\left[\begin{array}{cc}\widehat{\mathbf{A}}_{11} & \widehat{\mathbf{A}}_{12} \\ -\widehat{\mathbf{A}}_{12} & -\widehat{\mathbf{A}}_{11}\end{array}\right]$

with

$\widehat{\mathbf{A}}_{11}=\mathbf{M}^{\frac{1}{2}} \mathbf{W}^{\frac{1}{2}}\left(\mathbf{S}_{+}-\mathbf{W}^{-1}\right) \mathbf{M}^{\frac{1}{2}} \mathbf{W}^{\frac{1}{2}}$,

$\widehat{\mathbf{A}}_{12}=\mathbf{M}^{\frac{1}{2}} \mathbf{W}^{\frac{1}{2}} \mathbf{S}_{-} \mathbf{M}^{\frac{1}{2}} \mathbf{W}^{\frac{1}{2}}$, and let us introduce the symmetric matrices

$\widehat{\mathbf{A}}_{+}=\widehat{\mathbf{A}}_{11}+\widehat{\mathbf{A}}_{12}=\mathbf{M}^{\frac{1}{2}} \mathbf{W}^{\frac{1}{2}}\left(\mathbf{S}_{+}+\mathbf{S}_{-}\right) \mathbf{M}^{\frac{1}{2}} \mathbf{W}^{\frac{1}{2}}-\mathbf{M}$

$\widehat{\mathbf{A}}_{-}=\widehat{\mathbf{A}}_{11}-\widehat{\mathbf{A}}_{12}=\mathbf{M}^{\frac{1}{2}} \mathbf{W}^{\frac{1}{2}}\left(\mathbf{S}_{+}-\mathbf{S}_{-}\right) \mathbf{M}^{\frac{1}{2}} \mathbf{W}^{\frac{1}{2}}-\mathbf{M}$.

Further, let us define the matrices $\widehat{\mathbf{Q}}_{+}$and $\widehat{\mathbf{Q}}_{-}$through the factorizations

$\widehat{\mathbf{A}}_{-} \widehat{\mathbf{Q}}_{-}=\widehat{\mathbf{Q}}_{+} \Lambda$,

$\widehat{\mathbf{A}}_{+} \widehat{\mathbf{Q}}_{+}=\widehat{\mathbf{Q}}_{-} \Lambda$,

yielding

$\left(\widehat{\mathbf{A}}_{-} \widehat{\mathbf{A}}_{+}\right) \widehat{\mathbf{Q}}_{+}=\widehat{\mathbf{Q}}_{+} \Lambda^{2}$,

and

$\left(\widehat{\mathbf{A}}_{+} \widehat{\mathbf{A}}_{-}\right) \widehat{\mathbf{Q}}_{-}=\widehat{\mathbf{Q}}_{-} \Lambda^{2}$,

for $\Lambda=\left[\lambda_{k}\right]$. If $\widehat{\mathbf{Q}}_{+}, \widehat{\mathbf{Q}}_{-}$and $\Lambda$ are known, the desired eigendecomposition is

$\widehat{\mathbf{A}}=\left[\begin{array}{ll}\widehat{\mathbf{V}}_{+} & \widehat{\mathbf{V}}_{-} \\ \widehat{\mathbf{V}}_{-} & \widehat{\mathbf{V}}_{+}\end{array}\right]\left[\begin{array}{cc}\Lambda & \mathbf{0} \\ \mathbf{0} & -\Lambda\end{array}\right]\left[\begin{array}{cc}\widehat{\mathbf{V}}_{+} & \widehat{\mathbf{V}}_{-} \\ \widehat{\mathbf{V}}_{-} & \widehat{\mathbf{V}}_{+}\end{array}\right]^{-1}$,

where

$\widehat{\mathbf{V}}_{+}=\frac{1}{2}\left(\widehat{\mathbf{Q}}_{+}+\widehat{\mathbf{Q}}_{-}\right)$,

$\widehat{\mathbf{V}}_{-}=\frac{1}{2}\left(\widehat{\mathbf{Q}}_{+}-\widehat{\mathbf{Q}}_{-}\right)$.

If the systems of normalized right and left eigenvectors are required, we first compute $a_{k}=\left\|\widehat{\mathbf{v}}_{k}^{-}\right\|^{2}-\left\|\widehat{\mathbf{v}}_{k}^{+}\right\|^{2}$, and then apply the transformation rules

$\frac{1}{\sqrt{\left|a_{k}\right|}} \widehat{\mathbf{v}}_{k}^{ \pm} \rightarrow \widehat{\mathbf{v}}_{k}^{ \pm}$and $\overline{\mathbf{v}}_{k}^{ \pm}=\operatorname{sign}\left(a_{k}\right) \widehat{\mathbf{v}}_{k}^{ \pm}$

to construct the matrices $\widehat{\mathbf{V}}_{ \pm}$and $\overline{\mathbf{V}}_{ \pm}$.

The spectral decomposition of $\hat{\mathbf{A}}$ can be obtained by one of the following methods: direct decomposition of the asymmetric matrix $\widehat{\mathbf{A}}_{-} \widehat{\mathbf{A}}_{+}$[14], the square-root decomposition [18], and the Cholesky decomposition [30]. Before proceeding, we make the change of variables

$\mathbf{A}_{-}=-\widehat{\mathbf{A}}_{-}$,

$\mathbf{A}_{+}=-\widehat{\mathbf{A}}_{+}$,

and note that by virtue of (330) and (331), the matrix $\mathbf{A}_{-}$is symmetric and positive definite, while the matrix $\mathbf{A}_{+}$is symmetric and non-negative definite.

\section{Direct decomposition method}

The direct decomposition method involves the following steps:

1. Compute $\mathscr{A}_{+}=\mathbf{A}_{-} \mathbf{A}_{+}$, and determine the eigensystem $\left\{\mu_{k}, \widehat{\mathbf{q}}_{k}^{+}\right\}_{k=\overline{1, N}}$ of the matrix $\mathscr{A}_{+}$, i.e., $\mathscr{A}_{+} \widehat{\mathbf{q}}_{k}^{+}=\mu_{k} \widehat{\mathbf{q}}_{k}^{+}$. 
2. If a linearization of the radiative transfer model is required, normalize the vectors $\widehat{\mathbf{q}}_{k}^{+}$for $k=1, \ldots, N$.

3 . For the nonconservative case $\left(\mu_{N} \neq 0\right)$, compute the eigenvectors of the matrix $\mathscr{A}_{-}=\mathbf{A}_{+} \mathbf{A}_{-}$, i.e.,

$$
\widehat{\mathbf{q}}_{k}^{-}=-\frac{1}{\sqrt{\mu_{k}}} \mathbf{A}_{+} \widehat{\mathbf{q}}_{k}^{+}, k=1, \ldots, N
$$

4. Set

$$
\Lambda=\left[\lambda_{k}\right], \lambda_{k}=\sqrt{\mu_{k}},
$$

and

$$
\begin{aligned}
& \widehat{\mathbf{Q}}_{+}=\left[\widehat{\mathbf{q}}_{k}^{+}\right], \\
& \widehat{\mathbf{Q}}_{-}=\left[\widehat{\mathbf{q}}_{k}^{-}\right] .
\end{aligned}
$$

\section{Square-root method}

The square-root method involves the following steps:

1. Compute a singular value decomposition of the symmetric and positive definite matrix $\mathbf{A}_{-}$, i.e.,

$\mathbf{A}_{-}=\mathbf{U} \Sigma_{\mathrm{a}} \mathbf{U}^{T}$,

and the square root matrices

$$
\begin{aligned}
\mathbf{A}_{-}^{\frac{1}{2}} & =\mathbf{U} \Sigma_{\mathrm{a}}^{\frac{1}{2}} \mathbf{U}^{T}, \\
\mathbf{A}_{-}^{-\frac{1}{2}} & =\mathbf{U} \Sigma_{\mathrm{a}^{-\frac{1}{2}}} \mathbf{U}^{T} .
\end{aligned}
$$

2. Construct the matrix

$\mathbf{Z}=\mathbf{A}_{-}^{\frac{1}{2}} \mathbf{A}_{+} \mathbf{A}_{-}^{\frac{1}{2}}$,

and compute a singular value decomposition of the symmetric and non-negative definite matrix $\mathbf{Z}$, i.e.,

$\mathbf{Z}=\mathbf{V} \Sigma_{\mathbf{z}} \mathbf{V}^{T}$

3. Set

$$
\Lambda=\Sigma_{\mathrm{z}}^{\frac{1}{2}}
$$

and compute

$$
\begin{aligned}
\widehat{\mathbf{Q}}_{+} & =\mathbf{A}_{-}^{\frac{1}{2}} \mathbf{V}, \\
\widehat{\mathbf{Q}}_{+}^{-T} & =\mathbf{A}_{-}^{-\frac{1}{2}} \mathbf{V}, \\
\widehat{\mathbf{Q}}_{-} & =-\widehat{\mathbf{Q}}_{+}^{-T} \boldsymbol{\Lambda} .
\end{aligned}
$$

To justify this algorithm we note that

$$
\mathbf{A}_{-} \mathbf{A}_{+}=\mathbf{A}_{-}^{\frac{1}{2}}\left(\mathbf{A}_{-}^{\frac{1}{2}} \mathbf{A}_{+} \mathbf{A}_{-}^{\frac{1}{2}}\right) \mathbf{A}_{-}^{-\frac{1}{2}}=\mathbf{A}_{-}^{\frac{1}{2}} \mathbf{V} \Sigma_{\mathbf{z}} \mathbf{V}^{T} \mathbf{A}_{-}^{-\frac{1}{2}}=\widehat{\mathbf{Q}}_{+} \Lambda^{2} \widehat{\mathbf{Q}}_{+}^{-1},
$$

which is equivalent to (326).

\section{Cholesky method}

The Cholesky method involves the following steps:

1. Compute the Cholesky factorization of the symmetric and non-negative definite matrix $\mathbf{A}_{+}$, i.e.,

$$
\mathbf{A}_{+}=\mathbf{R}^{T} \mathbf{R} \text {. }
$$

2. Construct the matrix

$$
\mathbf{Z}=\mathbf{R A}_{-} \mathbf{R}^{T},
$$

and compute a singular value decomposition of the symmetric and non-negative definite matrix $\mathbf{Z}$, i.e.,

$\mathbf{Z}=\mathbf{V} \Sigma_{\mathbf{z}} \mathbf{V}^{T}$

3. Set

$\Lambda=\Sigma_{\mathrm{z}}^{\frac{1}{2}}$

and for the nonconservative case $\left(\Lambda^{-1}\right.$ and $\mathbf{R}^{-1}$ exist), compute

$\widehat{\mathbf{Q}}_{-}=-\mathbf{R}^{T} \mathbf{V} \Lambda^{-1}$,

and

$$
\begin{aligned}
\widehat{\mathbf{Q}}_{+} & =\mathbf{R}^{-1} \mathbf{V}, \\
\widehat{\mathbf{Q}}_{+}^{-T} & =-\widehat{\mathbf{Q}}_{-} \Lambda .
\end{aligned}
$$

To justify the algorithm, we note that

$\widehat{\mathbf{Q}}_{+} \Lambda^{2}=\mathbf{R}^{-1} \mathbf{V} \Sigma_{z}=\mathbf{R}^{-1} \mathbf{Z V}=\mathbf{R}^{-1} \mathbf{R} \mathbf{A}_{-} \mathbf{R}^{T} \mathbf{R} \mathbf{R}^{-1} \mathbf{V}=\mathbf{A}_{-} \mathbf{A}_{+} \widehat{\mathbf{Q}}_{+}$,

which is equivalent to (326).

In summary,

1. in the direct decomposition method, only one eigenvalue problem for the asymmetric matrix $\widehat{\mathbf{A}}_{-} \widehat{\mathbf{A}}_{+}$is solved,

2 . in the square-root method, two eigenvalue problems for the symmetric and positive definite matrix $\mathbf{A}_{-}$and the symmetric and non-negative definite matrix $\mathbf{A}_{-}^{1 / 2} \mathbf{A}_{+} \mathbf{A}_{-}^{1 / 2}$ are solved, and

3. in the Cholesky method, the matrix $\mathbf{A}_{+}$is first factorized as $\mathbf{A}_{+}=\mathbf{R}^{T} \mathbf{R}$, and then, an eigenvalue problem for the symmetric and non-negative definite matrix $\mathbf{R A}_{-} \mathbf{R}^{T}$ is solved.

Although the computation of the eigendecomposition of $\widehat{\mathbf{A}}$ by the square-root method is more time consuming than the other two methods, it is computationally stable in the limit $\omega \rightarrow 1$, and even in the conservative case $\omega=1$.

\section{Appendix 3. Conservative scattering}

In the conservative case and the azimuthal mode $m=0$, the smallest eigenvalues $\pm \lambda_{N}$ vanish. The two corresponding eigenvectors merge into one, which is not normalizable. The idea is to introduce two additional terms to replace those 
that are lost in expansion (40) when $k=N$. In fact, the case of conservative scattering is only of pure theoretical interest. The reason is that in practical numerical simulations, a conservative scattering problem can be modeled by considering the limit $\omega \rightarrow 1$, i.e., by setting $\omega=1-\varepsilon$, with $\varepsilon$ sufficiently small. Even in the case $\omega=1$, the smallest computed eigenvalue is not exactly zero (due to rounding errors), and the computation of the spectral decomposition of the layer matrix be means of the square root method of Appendix 2 is a stable process. In spite of these practical arguments we decide to include this case in our analysis.

Before proceeding we make some general comments. Let A be a matrix with $n-2$ real eigenvalues $\lambda_{k}$ with the geometric multiplicities $m_{k}=1, k=1, \ldots, n-2$, and let $\lambda_{n}$ be an eigenvalue with the geometric multiplicity $m_{n}=2$. For the eigenvalue $\lambda_{n}$, the solution of the differential equation

$\frac{\mathrm{d} \mathbf{i}}{\mathrm{d} \tau}(\tau)=-\mathbf{A i}(\tau)$

is seek as a linear combination of characteristic solutions

$\mathbf{i}(\tau)=\alpha \mathbf{w}_{0} \mathrm{e}^{-\lambda_{n} \tau}+\beta\left(\mathbf{w}_{1}+\mathbf{w}_{0} \tau\right) \mathrm{e}^{-\lambda_{n} \tau}$,

where $\mathbf{w}_{0}$ and $\mathbf{w}_{1}$ are determined by inserting each characteristic solution into the differential equation. By doing this, we obtain

$$
\begin{aligned}
\left(\mathbf{A}-\lambda_{n} \mathbf{I}_{n}\right) \mathbf{w}_{0} & =0, \\
\left(\mathbf{A}-\lambda_{n} \mathbf{I}_{n}\right) \mathbf{w}_{1} & =-\mathbf{w}_{0}, \\
\left(\mathbf{A}-\lambda_{n} \mathbf{I}_{n}\right)^{2} \mathbf{w}_{1} & =0 .
\end{aligned}
$$

Defining the null spaces $\mathscr{N}_{\lambda_{n}}^{1}=\mathscr{N}\left(\mathbf{A}-\lambda_{n} \mathbf{I}_{n}\right)$ and $\mathscr{N}_{\lambda_{n}}^{2}=$ $\mathscr{N}\left(\left(\mathbf{A}-\lambda_{n} \mathbf{I}_{n}\right)^{2}\right)$, and noting that $\mathscr{N}_{\lambda_{n}}^{1} \subset \mathscr{N}_{\lambda_{n}}^{2}=\mathscr{M}_{\lambda_{n}}$, where $\mathscr{M}_{\lambda_{n}}$ is the main space of the eigenvalue $\lambda_{n}$ so that $\mathbb{R}^{n}=$

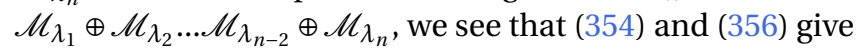

$\mathbf{w}_{0} \in \mathscr{N}\left(\mathbf{A}-\lambda_{n} \mathbf{I}_{n}\right)$,

$\mathbf{w}_{1} \in \mathscr{N}\left(\left(\mathbf{A}-\lambda_{n} \mathbf{I}_{n}\right)^{2}\right) \backslash \mathscr{N}\left(\mathbf{A}-\lambda_{n} \mathbf{I}_{n}\right)$,

and further, $\mathscr{N}_{\lambda_{n}}^{1}=\operatorname{span}\left\{\mathbf{w}_{0}\right\}$ and $\mathscr{N}_{\lambda_{n}}^{2}=\operatorname{span}\left\{\mathbf{w}_{0}, \mathbf{w}_{1}\right\}$.

\section{Basic results}

In the conservative case and the azimuthal mode $m=0$, we have $\lambda_{N}=0$, and so, $\lambda_{N}=0$ is an eigenvalue with the geometric multiplicity $m_{N}=2$. The right and left eigenvectors of the matrix $\widehat{\mathbf{A}}$ are constructed as follows:

1. We assume that the null spaces $\mathscr{N}(\widehat{\mathbf{A}})$ and $\mathscr{N}\left(\widehat{\mathbf{A}}^{2}\right) \backslash$ $\mathscr{N}(\widehat{\mathbf{A}})$ are spanned by the right eigenvectors $\left[\begin{array}{l}\mathbf{w}_{0} \\ \mathbf{w}_{0}\end{array}\right]$ and $\left[\begin{array}{c}\mathbf{w}_{1} \\ -\mathbf{w}_{1}\end{array}\right]$, respectively, i.e.,

$$
\begin{array}{r}
\mathscr{N}(\widehat{\mathbf{A}})=\operatorname{span}\left\{\left[\begin{array}{c}
\mathbf{w}_{0} \\
\mathbf{w}_{0}
\end{array}\right]\right\}, \\
\mathscr{N}\left(\widehat{\mathbf{A}}^{2}\right) \backslash \mathscr{N}(\widehat{\mathbf{A}})=\operatorname{span}\left\{\left[\begin{array}{c}
\mathbf{w}_{1} \\
-\mathbf{w}_{1}
\end{array}\right]\right\},
\end{array}
$$

and that the null-spaces $\mathscr{N}\left(\widehat{\mathbf{A}}^{T}\right)$ and $\mathscr{N}\left(\left(\widehat{\mathbf{A}}^{2}\right)^{T}\right) \backslash \mathscr{N}\left(\widehat{\mathbf{A}}^{T}\right)$ are spanned by the left eigenvectors $\left[\begin{array}{c}\mathbf{w}_{0} \\ -\mathbf{w}_{0}\end{array}\right]$ and $\left[\begin{array}{l}\mathbf{w}_{1} \\ \mathbf{w}_{1}\end{array}\right]$, respectively, i.e.,

$$
\begin{aligned}
\mathscr{N}\left(\widehat{\mathbf{A}}^{T}\right) & =\operatorname{span}\left\{\left[\begin{array}{c}
\mathbf{w}_{0} \\
-\mathbf{w}_{0}
\end{array}\right]\right\}, \\
\mathscr{N}\left(\left(\widehat{\mathbf{A}}^{2}\right)^{T}\right) \backslash \mathscr{N}\left(\widehat{\mathbf{A}}^{T}\right) & =\operatorname{span}\left\{\left[\begin{array}{c}
\mathbf{w}_{1} \\
\mathbf{w}_{1}
\end{array}\right]\right\} .
\end{aligned}
$$

Thus, we have

$$
\begin{gathered}
\mathscr{N}\left(\widehat{\mathbf{A}}^{2}\right)=\operatorname{span}\left\{\left[\begin{array}{c}
\mathbf{w}_{0} \\
\mathbf{w}_{0}
\end{array}\right],\left[\begin{array}{c}
\mathbf{w}_{1} \\
-\mathbf{w}_{1}
\end{array}\right]\right\}, \\
\mathscr{N}\left(\left(\widehat{\mathbf{A}}^{2}\right)^{T}\right)=\operatorname{span}\left\{\left[\begin{array}{c}
\mathbf{w}_{0} \\
-\mathbf{w}_{0}
\end{array}\right],\left[\begin{array}{c}
\mathbf{w}_{1} \\
\mathbf{w}_{1}
\end{array}\right]\right\} .
\end{gathered}
$$

2. We impose that the vectors $\mathbf{w}_{0}$ and $\mathbf{w}_{1}$ are related through the relation (cf. (355))

$$
\widehat{\mathbf{A}}\left[\begin{array}{c}
\mathbf{w}_{1} \\
-\mathbf{w}_{1}
\end{array}\right]=-\left[\begin{array}{l}
\mathbf{w}_{0} \\
\mathbf{w}_{0}
\end{array}\right] .
$$

Let us normalize the vectors $\mathbf{w}_{0}$ and $\mathbf{w}_{1}$ according to the transformations

$\frac{1}{a_{0}} \mathbf{w}_{0} \rightarrow \mathbf{w}_{0}$ and $\frac{1}{a_{0}} \mathbf{w}_{1} \rightarrow \mathbf{w}_{1}$,

where the normalization constant $a_{0}$ is given by $a_{0}=$ $\sqrt{2 \mathbf{w}_{0}^{T} \mathbf{w}_{1}}$. Then, the following results hold:

1. The right eigenvector spanning $\mathscr{N}(\widehat{\mathbf{A}})$ is orthogonal to the right eigenvector spanning $\mathscr{N}\left(\widehat{\mathbf{A}}^{2}\right) \backslash \mathscr{N}(\widehat{\mathbf{A}})$. The same result is valid for the left eigenvectors spanning $\mathscr{N}\left(\widehat{\mathbf{A}}^{T}\right)$ and $\mathscr{N}\left(\left(\widehat{\mathbf{A}}^{2}\right)^{T}\right) \backslash \mathscr{N}\left(\widehat{\mathbf{A}}^{T}\right)$, i.e.,

$$
\left[\begin{array}{c}
\mathbf{w}_{0} \\
\mathbf{w}_{0}
\end{array}\right]^{T}\left[\begin{array}{c}
\mathbf{w}_{1} \\
-\mathbf{w}_{1}
\end{array}\right]=\left[\begin{array}{c}
\mathbf{w}_{0} \\
-\mathbf{w}_{0}
\end{array}\right]^{T}\left[\begin{array}{l}
\mathbf{w}_{1} \\
\mathbf{w}_{1}
\end{array}\right]=0 .
$$

2. The right eigenvector spanning $\mathscr{N}(\widehat{\mathbf{A}})$ is orthogonal to the left eigenvector spanning $\mathscr{N}\left(\widehat{\mathbf{A}}^{T}\right)$. The same result is valid for the right and left eigenvectors spanning $\mathscr{N}\left(\widehat{\mathbf{A}}^{2}\right) \backslash \mathscr{N}(\widehat{\mathbf{A}})$ and $\mathscr{N}\left(\left(\widehat{\mathbf{A}}^{2}\right)^{T}\right) \backslash \mathscr{N}\left(\widehat{\mathbf{A}}^{T}\right)$, respectively, i.e.,

$$
\left[\begin{array}{c}
\mathbf{w}_{0} \\
\mathbf{w}_{0}
\end{array}\right]^{T}\left[\begin{array}{c}
\mathbf{w}_{0} \\
-\mathbf{w}_{0}
\end{array}\right]=\left[\begin{array}{c}
\mathbf{w}_{1} \\
-\mathbf{w}_{1}
\end{array}\right]^{T}\left[\begin{array}{l}
\mathbf{w}_{1} \\
\mathbf{w}_{1}
\end{array}\right]=0 .
$$

3. The right eigenvector spanning $\mathscr{N}(\widehat{\mathbf{A}})$ is co-linear with the left eigenvector spanning $\mathscr{N}\left(\left(\widehat{\mathbf{A}}^{2}\right)^{T}\right) \backslash \mathscr{N}\left(\widehat{\mathbf{A}}^{T}\right)$. The same result is valid for the left and right eigenvectors spanning $\mathscr{N}\left(\widehat{\mathbf{A}}^{T}\right)$ and $\mathscr{N}\left(\widehat{\mathbf{A}}^{2}\right) \backslash \mathscr{N}(\widehat{\mathbf{A}})$, respectively, i.e.,

$$
\left[\begin{array}{l}
\mathbf{w}_{0} \\
\mathbf{w}_{0}
\end{array}\right]^{T}\left[\begin{array}{l}
\mathbf{w}_{1} \\
\mathbf{w}_{1}
\end{array}\right]=\left[\begin{array}{c}
\mathbf{w}_{0} \\
-\mathbf{w}_{0}
\end{array}\right]^{T}\left[\begin{array}{c}
\mathbf{w}_{1} \\
-\mathbf{w}_{1}
\end{array}\right]=1 .
$$


4. The left eigenvectors $\left[\begin{array}{c}\mathbf{w}_{0} \\ -\mathbf{w}_{0}\end{array}\right]$ and $\left[\begin{array}{l}\mathbf{w}_{1} \\ \mathbf{w}_{1}\end{array}\right]$ spanning $\mathscr{N}\left(\left(\widehat{\mathbf{A}}^{2}\right)^{T}\right)$ are orthogonal to the right eigenvectors $\left[\begin{array}{c}\widehat{\mathbf{v}}_{k}^{+} \\ \widehat{\mathbf{v}}_{k}^{-}\end{array}\right]$corresponding to $\lambda_{k}$, i.e.,

$\left[\begin{array}{c}\mathbf{w}_{0} \\ -\mathbf{w}_{0}\end{array}\right]^{T}\left[\begin{array}{c}\widehat{\mathbf{v}}_{k}^{+} \\ \widehat{\mathbf{v}}_{k}^{-}\end{array}\right]=\left[\begin{array}{c}\mathbf{w}_{1} \\ \mathbf{w}_{1}\end{array}\right]^{T}\left[\begin{array}{c}\widehat{\mathbf{v}}_{k}^{+} \\ \widehat{\mathbf{v}}_{k}^{-}\end{array}\right]=0$,

and to the right eigenvectors $\left[\begin{array}{c}\widehat{\mathbf{v}}_{k}^{-} \\ \widehat{\mathbf{v}}_{k}^{+}\end{array}\right]$corresponding to $-\lambda_{k}$, i.e.,

$\left[\begin{array}{c}\mathbf{w}_{0} \\ -\mathbf{w}_{0}\end{array}\right]^{T}\left[\begin{array}{c}\widehat{\mathbf{v}}_{k}^{-} \\ \widehat{\mathbf{v}}_{k}^{+}\end{array}\right]=\left[\begin{array}{c}\mathbf{w}_{1} \\ \mathbf{w}_{1}\end{array}\right]^{T}\left[\begin{array}{c}\widehat{\mathbf{v}}_{k}^{-} \\ \widehat{\mathbf{v}}_{k}^{+}\end{array}\right]=0$

\section{Computation of the vectors $\mathbf{w}_{0}$ nd $\mathbf{w}_{1}$}

A method for computing the vectors $\mathbf{w}_{0}$ and $\mathbf{w}_{1}$ uses the equations solved by the flux $H$ and the $K$ integral in the case of conservative scattering [32]:

$\frac{\mathrm{d} H}{\mathrm{~d} \tau}\left(\tau, \mu_{0}\right)=0$,
$\frac{\mathrm{d} K}{\mathrm{~d} \tau}\left(\tau, \mu_{0}\right)=\left(1-\frac{\chi_{1}}{2}\right) H\left(\tau, \mu_{0}\right)$,

where

$H\left(\tau, \mu_{0}\right)=2 \pi \int_{-1}^{1} I_{0}\left(\tau, \mu,-\mu_{0}\right) \mu \mathrm{d} \mu-F_{0} \mu_{0} \mathrm{e}^{-\tau / \mu_{0}}$,

$K\left(\tau, \mu_{0}\right)=2 \pi \int_{-1}^{1} I_{0}\left(\tau, \mu,-\mu_{0}\right) \mu^{2} \mathrm{~d} \mu+F_{0} \mu_{0}^{2} \mathrm{e}^{-\tau / \mu_{0}}$.

Another method is the square-root method of Appendix 2, which we will now describe. In the conservative case and the azimuthal mode $m=0$, the singular value $\sigma_{N}$ of the matrix $\mathbf{Z}=\mathbf{V} \Sigma_{\mathbf{z}} \mathbf{V}^{T}$ is zero. Thus, we have $\lambda_{N}=\sqrt{\sigma_{N}}=0$ and $\mathscr{N}(\mathbf{Z})=$ $\operatorname{span}\left\{\mathbf{v}_{N}\right\}$, where $\mathbf{v}_{N}$ is the $N$ th column vector of $\mathbf{V}$. From the relation $\mathbf{Z} \mathbf{v}_{N}=0$ and the definition $\mathbf{Z}=\mathbf{A}_{-}^{1 / 2} \mathbf{A}_{+} \mathbf{A}_{-}^{1 / 2}$, we obtain $\mathbf{A}_{-}^{1 / 2} \mathbf{A}_{+} \mathbf{A}_{-}^{1 / 2} \mathbf{v}_{N}=0$. Since $\mathbf{A}_{-}$is positive definite, it follows that $\mathbf{A}_{+} \mathbf{A}_{-}^{1 / 2} \mathbf{v}_{N}=0$. Further, setting $\widehat{\mathbf{q}}_{N}^{+}=\mathbf{A}_{-}^{1 / 2} \mathbf{v}_{N}$ (cf. (342)), we get $\mathbf{A}_{+} \widehat{\mathbf{q}}_{N}^{+}=0$, or equivalently, $\widehat{\mathbf{q}}_{N}^{+} \in \mathscr{N}\left(\mathbf{A}_{+}\right)$. On the other hand, from $\widehat{\mathbf{Q}}_{-}=-\mathbf{A}_{-}^{-1} \widehat{\mathbf{Q}}_{+} \Lambda$, we obtain $\widehat{\mathbf{q}}_{N}^{-}=-\lambda_{N} \mathbf{A}_{-}^{-1} \widehat{\mathbf{q}}_{N}^{+}=0$, yielding

$\widehat{\mathbf{v}}_{N}^{+}=\widehat{\mathbf{v}}_{N}^{-}=\frac{1}{2} \widehat{\mathbf{q}}_{N}^{+}$

Thus, the right eigenvectors of $\widehat{\mathbf{A}}$ corresponding to $\lambda_{N}$ and $-\lambda_{N}$ merge into one, i.e.,

$\left[\begin{array}{c}\widehat{\mathbf{v}}_{N}^{+} \\ \widehat{\mathbf{v}}_{N}^{-}\end{array}\right]=\left[\begin{array}{c}\widehat{\mathbf{v}}_{N}^{-} \\ \widehat{\mathbf{v}}_{N}^{+}\end{array}\right]=\frac{1}{2}\left[\begin{array}{c}\widehat{\mathbf{q}}_{N}^{+} \\ \widehat{\mathbf{q}}_{N}^{+}\end{array}\right]$

From $\widehat{\mathbf{q}}_{N}^{+} \in \mathscr{N}\left(\mathbf{A}_{+}\right)$, it follows that

$\widehat{\mathbf{A}}\left[\begin{array}{c}\widehat{\mathbf{q}}_{N}^{+} \\ \widehat{\mathbf{q}}_{N}^{+}\end{array}\right]=\left[\begin{array}{c}-\mathbf{A}_{+} \widehat{\mathbf{q}}_{N}^{+} \\ \mathbf{A}_{+} \widehat{\mathbf{q}}_{N}^{+}\end{array}\right]=0$,

and for the first eigenvector

$\left[\begin{array}{l}\mathbf{w}_{0} \\ \mathbf{w}_{0}\end{array}\right] \in \mathscr{N}(\widehat{\mathbf{A}})$,

we infer that $\mathbf{w}_{0}$ is given by

$\mathbf{w}_{0}=\widehat{\mathbf{q}}_{N}^{+}$.

For the second eigenvector

$\left[\begin{array}{c}\mathbf{w}_{1} \\ -\mathbf{w}_{1}\end{array}\right] \in \mathscr{N}\left(\widehat{\mathbf{A}}^{2}\right) \backslash \mathscr{N}(\widehat{\mathbf{A}})$,

we compute $\mathbf{w}_{1}$ such that the equation

$\widehat{\mathbf{A}}\left[\begin{array}{c}\mathbf{w}_{1} \\ -\mathbf{w}_{1}\end{array}\right]=-\left[\begin{array}{c}\mathbf{w}_{0} \\ \mathbf{w}_{0}\end{array}\right]$

is fullfield. Accounting of

$\widehat{\mathbf{A}}\left[\begin{array}{c}\mathbf{w}_{1} \\ -\mathbf{w}_{1}\end{array}\right]=\left[\begin{array}{c}-\mathbf{A}_{-} \mathbf{w}_{1} \\ -\mathbf{A}_{-} \mathbf{w}_{1}\end{array}\right]$,

and taking into account that $\mathbf{A}_{-}$is invertible, we deduce that for

$\mathbf{w}_{1}=\mathbf{A}_{-}^{-1} \mathbf{w}_{0}=\mathbf{A}_{-}^{-1} \widehat{\mathbf{q}}_{N}^{+}$,

equation (375) holds true. Thus, $\mathbf{w}_{0}$ and $\mathbf{w}_{1}$ are given by (374) and (377), respectively.

Analytical formulas for conservative scattering

Using (365)-(370), we find that the Jordan form representation of the layer matrix is

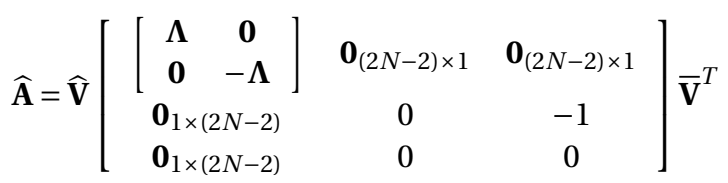

with

$\widehat{\mathbf{V}}=\left[\left[\begin{array}{cc}\widehat{\mathbf{V}}_{+} & \widehat{\mathbf{V}}_{-} \\ \widehat{\mathbf{V}}_{-} & \widehat{\mathbf{V}}_{+}\end{array}\right],\left[\begin{array}{c}\mathbf{w}_{0} \\ \mathbf{w}_{0}\end{array}\right],\left[\begin{array}{c}\mathbf{w}_{1} \\ -\mathbf{w}_{1}\end{array}\right]\right]$,

$\overline{\mathbf{V}}=\left[\left[\begin{array}{cc}-\overline{\mathbf{V}}_{+} & \overline{\mathbf{V}}_{-} \\ \overline{\mathbf{V}}_{-} & -\overline{\mathbf{V}}_{+}\end{array}\right],\left[\begin{array}{c}\mathbf{w}_{1} \\ \mathbf{w}_{1}\end{array}\right],\left[\begin{array}{c}\mathbf{w}_{0} \\ -\mathbf{w}_{0}\end{array}\right]\right]$,

$\boldsymbol{\Lambda}=\operatorname{diag}\left[\lambda_{1}, \ldots, \lambda_{N-1}\right] \in \mathbb{R}^{(N-1) \times(N-1)}, \widehat{\mathbf{V}}_{ \pm}=\left[\widehat{\mathbf{v}}_{1}^{ \pm}, \ldots, \widehat{\mathbf{v}}_{N-1}^{ \pm}\right] \in$ $\mathbb{R}^{N \times(N-1)}$, and $\overline{\mathbf{V}}_{ \pm}=\left[\overline{\mathbf{v}}_{1}^{ \pm}, \ldots, \overline{\mathbf{v}}_{N-1}^{ \pm}\right] \in \mathbb{R}^{N \times(N-1)}$, while by virtue of the matrix identity

$\mathrm{e}^{-\tau\left[\begin{array}{cc}0 & -1 \\ 0 & 0\end{array}\right]}=\left[\begin{array}{ll}1 & \tau \\ 0 & 1\end{array}\right]$,

the Jordan form representation of the matrix exponential is

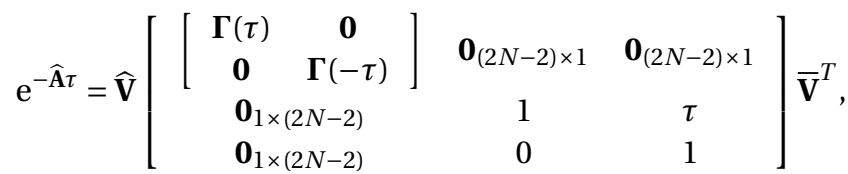


with $\boldsymbol{\Gamma}(\tau)=\operatorname{diag}\left[\mathrm{e}^{-\lambda_{1} \tau}, \ldots, \mathrm{e}^{-\lambda_{N-1} \tau}\right] \in \mathbb{R}^{(N-1) \times(N-1)}$.

Insertion of (379) and (380) in (378) and (382), gives a resolution of the layer matrix

$$
\begin{aligned}
\widehat{\mathbf{A}} & =-\left[\begin{array}{c}
\mathbf{w}_{0} \\
\mathbf{w}_{0}
\end{array}\right]\left[\begin{array}{c}
\mathbf{w}_{0} \\
-\mathbf{w}_{0}
\end{array}\right]^{T} \\
& +\sum_{k=1}^{N-1} \lambda_{k}\left[\begin{array}{c}
\widehat{\mathbf{v}}_{k}^{+} \\
\widehat{\mathbf{v}}_{k}^{-}
\end{array}\right]\left[\begin{array}{c}
-\overline{\mathbf{v}}_{k}^{+} \\
\overline{\mathbf{v}}_{k}^{-}
\end{array}\right]^{T}-\lambda_{k}\left[\begin{array}{c}
\widehat{\mathbf{v}}_{k}^{-} \\
\widehat{\mathbf{v}}_{k}^{+}
\end{array}\right]\left[\begin{array}{c}
\overline{\mathbf{v}}_{k}^{-} \\
-\overline{\mathbf{v}}_{k}^{+}
\end{array}\right]^{T},
\end{aligned}
$$

and of the matrix exponential

$$
\begin{aligned}
\mathrm{e}^{-\widehat{\mathbf{A}} \tau} & =\left[\begin{array}{l}
\mathbf{w}_{0} \\
\mathbf{w}_{0}
\end{array}\right]\left[\begin{array}{l}
\mathbf{w}_{1} \\
\mathbf{w}_{1}
\end{array}\right]^{T}+\left[\begin{array}{c}
\mathbf{w}_{1} \\
-\mathbf{w}_{1}
\end{array}\right]\left[\begin{array}{c}
\mathbf{w}_{0} \\
-\mathbf{w}_{0}
\end{array}\right]^{T}+\tau\left[\begin{array}{c}
\mathbf{w}_{0} \\
\mathbf{w}_{0}
\end{array}\right]\left[\begin{array}{c}
\mathbf{w}_{0} \\
-\mathbf{w}_{0}
\end{array}\right. \\
& +\sum_{k=1}^{N-1} \mathrm{e}^{-\lambda_{k} \tau}\left[\begin{array}{c}
\widehat{\mathbf{v}}_{k}^{+} \\
\widehat{\mathbf{v}}_{k}^{-}
\end{array}\right]\left[\begin{array}{c}
-\overline{\mathbf{v}}_{k}^{+} \\
\overline{\mathbf{v}}_{k}^{-}
\end{array}\right]^{T}+\mathrm{e}^{\lambda_{k} \tau}\left[\begin{array}{c}
\widehat{\mathbf{v}}_{k}^{-} \\
\widehat{\mathbf{v}}_{k}^{+}
\end{array}\right]\left[\begin{array}{c}
\overline{\mathbf{v}}_{k}^{-} \\
-\overline{\mathbf{v}}_{k}^{+}
\end{array}\right]^{T},
\end{aligned}
$$

respectively. Setting $\tau=0$ in (384) we obtain a resolution of the identity matrix

$$
\begin{aligned}
\mathbf{I}_{2 N} & =\left[\begin{array}{l}
\mathbf{w}_{0} \\
\mathbf{w}_{0}
\end{array}\right]\left[\begin{array}{l}
\mathbf{w}_{1} \\
\mathbf{w}_{1}
\end{array}\right]^{T}+\left[\begin{array}{c}
\mathbf{w}_{1} \\
-\mathbf{w}_{1}
\end{array}\right]\left[\begin{array}{c}
\mathbf{w}_{0} \\
-\mathbf{w}_{0}
\end{array}\right]^{T} \\
& +\sum_{k=1}^{N-1}\left[\begin{array}{c}
\widehat{\mathbf{v}}_{k}^{+} \\
\widehat{\mathbf{v}}_{k}^{-}
\end{array}\right]\left[\begin{array}{c}
-\overline{\mathbf{v}}_{k}^{+} \\
\overline{\mathbf{v}}_{k}^{-}
\end{array}\right]^{T}+\left[\begin{array}{c}
\widehat{\mathbf{v}}_{k}^{-} \\
\widehat{\mathbf{v}}_{k}^{+}
\end{array}\right]\left[\begin{array}{c}
\overline{\mathbf{v}}_{k}^{-} \\
-\overline{\mathbf{v}}_{k}^{+}
\end{array}\right]^{T},
\end{aligned}
$$

which further, gives

$$
\begin{aligned}
& \mathbf{w}_{0} \mathbf{w}_{1}^{T}+\mathbf{w}_{1} \mathbf{w}_{0}^{T}+\sum_{k=1}^{N-1} \widehat{\mathbf{v}}_{k}^{-} \overline{\mathbf{v}}_{k}^{-T}-\widehat{\mathbf{v}}_{k}^{+} \overline{\mathbf{v}}_{k}^{+T}=\mathbf{I}_{N}, \\
& \mathbf{w}_{0} \mathbf{w}_{1}^{T}-\mathbf{w}_{1} \mathbf{w}_{0}^{T}+\sum_{k=1}^{N-1} \widehat{\mathbf{v}}_{k}^{+} \overline{\mathbf{v}}_{k}^{-T}-\widehat{\mathbf{v}}_{k}^{-} \overline{\mathbf{v}}_{k}^{+T}=0 .
\end{aligned}
$$

Equations (378), (382), (383), and (384) are the counterparts of (27), (30), (40) and (42), respectively, for the conservative case. It should be pointed out that equations (383) and (384) have been derived by Waterman by employing different arguments.

By means of (382) and (384), the analytical formulas for nonconservative scattering can be extended to conservative scattering as follows:

1. The matrices $\mathbf{D}_{1}, \mathbf{D}_{0}$ and $\mathbf{D}_{\mathrm{b}}$ which enter in the layer equation (56) are given by

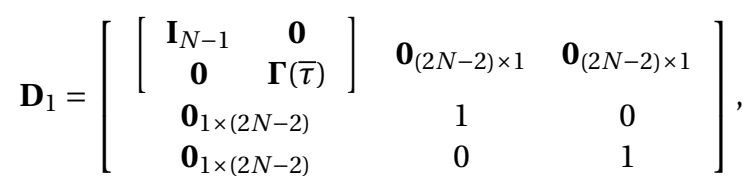

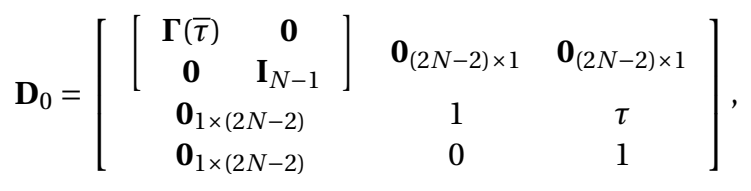

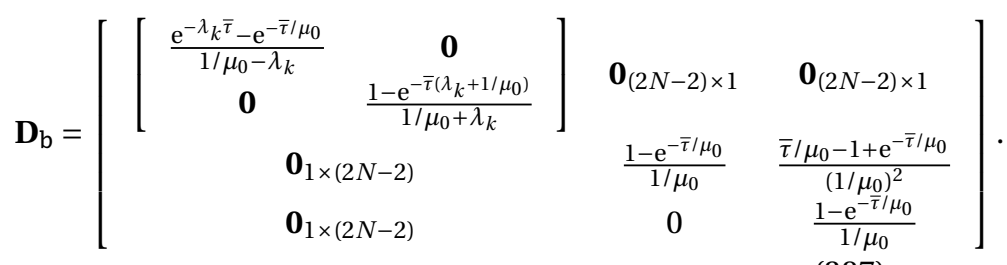

2. The solution representations (65) and (69) translate into

$$
\begin{aligned}
\widehat{\mathbf{i}}_{\mathrm{d}}(\tau) & =\alpha_{N}\left[\begin{array}{c}
\mathbf{w}_{0} \\
\mathbf{w}_{0}
\end{array}\right]+\beta_{N}\left(\left[\begin{array}{c}
\mathbf{w}_{1} \\
-\mathbf{w}_{1}
\end{array}\right]+\tau\left[\begin{array}{c}
\mathbf{w}_{0} \\
\mathbf{w}_{0}
\end{array}\right]\right) \\
& ]^{T}+\sum_{k=1}^{N-1} \alpha_{k} \mathrm{e}^{-\lambda_{k} \tau}\left[\begin{array}{c}
\widehat{\mathbf{v}}_{k}^{+} \\
\widehat{\mathbf{v}}_{k}^{-}
\end{array}\right]+\beta_{k} \mathrm{e}^{-\lambda_{k}(\bar{\tau}-\tau)}\left[\begin{array}{c}
\widehat{\mathbf{v}}_{k}^{-} \\
\widehat{\mathbf{v}}_{k}^{+}
\end{array}\right]+\hat{\mathbf{i}}_{\mathrm{p}}(\tau)
\end{aligned}
$$

and

$$
\begin{aligned}
\widehat{\mathbf{i}}_{\mathrm{d}}(\tau) & =\alpha_{N}\left[\begin{array}{c}
\mathbf{w}_{0} \\
\mathbf{w}_{0}
\end{array}\right]+\beta_{N}\left(\left[\begin{array}{c}
\mathbf{w}_{1} \\
-\mathbf{w}_{1}
\end{array}\right]+\tau\left[\begin{array}{c}
\mathbf{w}_{0} \\
\mathbf{w}_{0}
\end{array}\right]\right) \\
& +\sum_{k=1}^{N-1} \alpha_{k}\left(\mathrm{e}^{-\lambda_{k} \tau}\left[\begin{array}{c}
\widehat{\mathbf{v}}_{k}^{+} \\
\widehat{\mathbf{v}}_{k}^{-}
\end{array}\right]+\mathrm{e}^{-\lambda_{k}(\bar{\tau}-\tau)}\left[\begin{array}{c}
\widehat{\mathbf{v}}_{k}^{-} \\
\widehat{\mathbf{v}}_{k}^{+}
\end{array}\right]\right) \\
& +\beta_{k}\left(-\mathrm{e}^{-\lambda_{k} \tau}\left[\begin{array}{c}
\widehat{\mathbf{v}}_{k}^{+} \\
\widehat{\mathbf{v}}_{k}^{-}
\end{array}\right]+\mathrm{e}^{-\lambda_{k}(\bar{\tau}-\tau)}\left[\begin{array}{c}
\widehat{\mathbf{v}}_{k}^{-} \\
\widehat{\mathbf{v}}_{k}^{+}
\end{array}\right]\right)+\hat{\mathbf{i}}_{\mathrm{p}}(\tau),
\end{aligned}
$$

respectively.

3. The analogues of (108) and (109) which give the expressions of the transmission and reflection matrices, respectively, are

$\widehat{\mathbf{T}}=\overline{\mathbf{V}}_{-}^{-T} \boldsymbol{\Gamma}\left(\widehat{\mathbf{V}}_{-}^{0}-\widehat{\mathbf{V}}_{+}^{1} \boldsymbol{\Gamma} \overline{\mathbf{V}}_{+}^{T} \overline{\mathbf{V}}_{-}^{-T} \boldsymbol{\Gamma}\right)^{-1}$,

$\widehat{\mathbf{R}}=\left(\widehat{\mathbf{V}}_{+}^{0}-\widehat{\mathbf{V}}_{-}^{1} \boldsymbol{\Gamma} \overline{\mathbf{V}}_{+}^{T} \overline{\mathbf{V}}_{-}^{-T} \boldsymbol{\Gamma}\right)\left(\widehat{\mathbf{V}}_{-}^{0}-\widehat{\mathbf{V}}_{+}^{1} \boldsymbol{\Gamma} \overline{\mathbf{V}}_{+}^{T} \overline{\mathbf{V}}_{-}^{-T} \boldsymbol{\Gamma}\right)^{-1}$,

where now

$\boldsymbol{\Gamma}=\left[\begin{array}{cccc}\mathrm{e}^{-\lambda_{1} \bar{\tau}} & \ldots & 0 & 0 \\ & \cdots & & \\ 0 & \ldots & \mathrm{e}^{-\lambda_{N-1} \bar{\tau}} & 0 \\ 0 & \cdots & 0 & 1\end{array}\right]$

and

$\widehat{\mathbf{V}}_{ \pm}^{0}=\left[\widehat{\mathbf{v}}_{1}^{ \pm}, \ldots, \widehat{\mathbf{v}}_{N-1}^{ \pm}, \mathbf{w}_{0}\right]$,

$\widehat{\mathbf{V}}_{ \pm}^{1}=\left[\widehat{\mathbf{v}}_{1}^{ \pm}, \ldots, \widehat{\mathbf{v}}_{N-1}^{ \pm}, \pm \mathbf{w}_{1}\right]$,

$\overline{\mathbf{V}}_{+}=\left[\overline{\mathbf{v}}_{1}^{+}, \ldots, \overline{\mathbf{v}}_{N-1}^{+},-\mathbf{w}_{0}\right]$,

$\overline{\mathbf{V}}_{-}=\left[\overline{\mathbf{v}}_{1}^{-}, \ldots, \overline{\mathbf{v}}_{N-1}^{-}, \mathbf{w}_{1}+\tau \mathbf{w}_{0}\right]$.

\section{A special system of characteristic solutions}

We conclude this appendix by presenting a system of characteristic solutions which can be used for nonconservative and conservative scattering. It is of the form

$\widehat{\mathbf{i}}_{\mathrm{d}}(\tau)=\sum_{k=1}^{N} \alpha_{k} \mathbf{a}_{k}(\tau)+\beta_{k} \mathbf{b}_{k}(\tau)+\hat{\mathbf{i}}_{\mathrm{p}}(\tau)$, 
with

$$
\begin{aligned}
& \mathbf{a}_{k}(\tau)=\mathrm{e}^{-\lambda_{k} \tau}\left[\begin{array}{c}
\widehat{\mathbf{v}}_{k}^{+} \\
\widehat{\mathbf{v}}_{k}^{-}
\end{array}\right]+\mathrm{e}^{-\lambda_{k}(\bar{\tau}-\tau)}\left[\begin{array}{c}
\widehat{\mathbf{v}}_{k}^{-} \\
\widehat{\mathbf{v}}_{k}^{+}
\end{array}\right], \\
& \mathbf{b}_{k}(\tau)=\frac{1}{\lambda_{k}}\left(-\mathrm{e}^{-\lambda_{k} \tau}\left[\begin{array}{c}
\widehat{\mathbf{v}}_{k}^{+} \\
\widehat{\mathbf{v}}_{k}^{-}
\end{array}\right]+\mathrm{e}^{-\lambda_{k}(\bar{\tau}-\tau)}\left[\begin{array}{c}
\widehat{\mathbf{v}}_{k}^{-} \\
\widehat{\mathbf{v}}_{k}^{+}
\end{array}\right]\right) .
\end{aligned}
$$

Obviously, (394)-(396) are equivalent to (69) if we consider the transformation $\beta_{k} \rightarrow\left(1 / \lambda_{k}\right) \beta_{k}$. As a result, the reflection and transmission matrices $\widehat{\mathbf{R}}$ and $\widehat{\mathbf{T}}$ are given by (97) and (98), respectively. In the conservative case, $\mathbf{b}_{N}$ is singular and so is $\widehat{\mathbf{V}}_{-}-\widehat{\mathbf{V}}_{+} \boldsymbol{\Gamma}$ together with $\widehat{\mathbf{R}}$ and $\widehat{\mathbf{T}}$. However, these singularities are removable if we compute the eigenvectors $\widehat{\mathbf{v}}_{k}^{ \pm}$by the square-root method of Appendix 2. To show this, we consider (342)-(344), set $\mathbf{V}=\left[\mathbf{v}_{k}\right], \widehat{\mathbf{Q}}_{+}=\left[\widehat{\mathbf{q}}_{k}^{+}\right]$and $\mathbf{Q} \stackrel{\text { not }}{=} \widehat{\mathbf{Q}}_{+}^{-T}=\left[\mathbf{q}_{k}\right]$, and write in component form $\widehat{\mathbf{q}}_{k}^{+}=\mathbf{A}_{-}^{1 / 2} \mathbf{v}_{k}, \mathbf{q}_{k}=\mathbf{A}_{-}^{-1} \widehat{\mathbf{q}}_{k}^{+}=\mathbf{A}_{-}^{-1 / 2} \mathbf{v}_{k}$, and $\widehat{\mathbf{q}}_{k}^{-}=-\lambda_{k} \mathbf{q}_{k}$. As a result, (396) becomes

$$
\begin{aligned}
\mathbf{a}_{k} & =\frac{1}{2}\left[\mathrm{e}^{-\lambda_{k} \tau}+\mathrm{e}^{-\lambda_{k}(\bar{\tau}-\tau)}\right]\left[\begin{array}{c}
\widehat{\mathbf{q}}_{k}^{+} \\
\widehat{\mathbf{q}}_{k}^{+}
\end{array}\right] \\
& -\frac{1}{2} \lambda_{k}\left[\mathrm{e}^{-\lambda_{k} \tau}-\mathrm{e}^{-\lambda_{k}(\bar{\tau}-\tau)}\right]\left[\begin{array}{c}
\mathbf{q}_{k} \\
-\mathbf{q}_{k}
\end{array}\right], \\
\mathbf{b}_{k} & =-\frac{1}{2 \lambda_{k}}\left[\mathrm{e}^{-\lambda_{k} \tau}-\mathrm{e}^{-\lambda_{k}(\bar{\tau}-\tau)}\right]\left[\begin{array}{c}
\widehat{\mathbf{q}}_{k}^{+} \\
\widehat{\mathbf{q}}_{k}^{+}
\end{array}\right] \\
& +\frac{1}{2}\left[\mathrm{e}^{-\lambda_{k} \tau}+\mathrm{e}^{-\lambda_{k}(\bar{\tau}-\tau)}\right]\left[\begin{array}{c}
\mathbf{q}_{k} \\
-\mathbf{q}_{k}
\end{array}\right] .
\end{aligned}
$$

In the limit $\lambda_{N} \rightarrow 0$, we have $\widehat{\mathbf{q}}_{N}^{+}, \mathbf{q}_{N} \neq 0$, and $\widehat{\mathbf{q}}_{N}^{-}=0$. Accounting of $\mathbf{w}_{0}=\widehat{\mathbf{q}}_{N}^{+}, \mathbf{w}_{1}=\mathbf{A}_{-}^{-1} \widehat{\mathbf{q}}_{N}^{+}=\mathbf{q}_{N}$, and

$$
\lim _{\lambda_{N} \rightarrow 0}\left[-\frac{\mathrm{e}^{-\lambda_{N} \tau}-\mathrm{e}^{-\lambda_{N}(\bar{\tau}-\tau)}}{2 \lambda_{N}}\right]=\tau-\frac{\bar{\tau}}{2}
$$

we get

$$
\begin{aligned}
\lim _{\lambda_{N} \rightarrow 0}\left[\alpha_{N} \mathbf{a}_{N}(\tau)+\beta_{N} \mathbf{b}_{N}(\tau)\right] & =\left(\alpha_{N}-\frac{\bar{\tau}}{2} \beta_{N}\right)\left[\begin{array}{c}
\mathbf{w}_{0} \\
\mathbf{w}_{0}
\end{array}\right] \\
& +\beta_{N}\left(\tau\left[\begin{array}{l}
\mathbf{w}_{0} \\
\mathbf{w}_{0}
\end{array}\right]+\left[\begin{array}{c}
\mathbf{w}_{1} \\
-\mathbf{w}_{1}
\end{array}\right]\right),
\end{aligned}
$$

which is equivalent to the first two terms in (389). Coming to the reflection and transmission matrices, we use (329) and (344) with $\mathbf{Q}=\widehat{\mathbf{Q}}_{+}^{-T}$, to compute the matrix products which enter in (97) and (98) as

$\left(\widehat{\mathbf{V}}_{+}+\widehat{\mathbf{V}}_{-} \boldsymbol{\Gamma}\right)\left(\widehat{\mathbf{V}}_{-}+\widehat{\mathbf{V}}_{+} \boldsymbol{\Gamma}\right)^{-1}$

$=\left[\widehat{\mathbf{Q}}_{+}(\mathbf{I}+\boldsymbol{\Gamma})-\mathbf{Q} \Lambda(\mathbf{I}-\boldsymbol{\Gamma})\right]\left[\widehat{\mathbf{Q}}_{+}(\mathbf{I}+\boldsymbol{\Gamma})+\mathbf{Q} \Lambda(\mathbf{I}-\boldsymbol{\Gamma})\right]^{-1}$

and

$$
\begin{aligned}
& \left(\widehat{\mathbf{V}}_{+}-\widehat{\mathbf{V}}_{-} \boldsymbol{\Gamma}\right)\left(\widehat{\mathbf{V}}_{-}-\widehat{\mathbf{V}}_{+} \boldsymbol{\Gamma}\right)^{-1} \\
& =\left[\left(\widehat{\mathbf{V}}_{+}-\widehat{\mathbf{V}}_{-} \boldsymbol{\Gamma}\right) \boldsymbol{\Lambda}^{-1}\right]\left[\left(\widehat{\mathbf{V}}_{-}-\widehat{\mathbf{V}}_{+} \boldsymbol{\Gamma}\right) \boldsymbol{\Lambda}^{-1}\right]^{-1} \\
& =\left[\widehat{\mathbf{Q}}_{+}(\mathbf{I}-\boldsymbol{\Gamma}) \boldsymbol{\Lambda}^{-1}-\mathbf{Q}(\mathbf{I}+\boldsymbol{\Gamma})\right]\left[\widehat{\mathbf{Q}}_{+}(\mathbf{I}-\boldsymbol{\Gamma}) \boldsymbol{\Lambda}^{-1}+\mathbf{Q}(\mathbf{I}+\boldsymbol{\Gamma})\right]^{-1} .
\end{aligned}
$$

In the limit $\lambda_{N} \rightarrow 0$, the singularity in $(\mathbf{I}-\boldsymbol{\Gamma}) \boldsymbol{\Lambda}^{-1}$ is removable, i.e.,

$\lim _{\lambda_{N} \rightarrow 0} \frac{1-\mathrm{e}^{-\lambda_{N} \bar{\tau}}}{\lambda_{N}}=\bar{\tau}$, so that (97), (98), (400), and (401) give the expressions of $\widehat{\mathbf{R}}$ and $\widehat{\mathbf{T}}$ for nonconservative and conservative scattering. The system of characteristic solutions (394)-(396), as well as the reflection and transmission matrices of (97), (98), (400), and (401) have been used by Nakajima and Tanaka in their matrix formulation of the radiative transfer.

\section{Appendix 4. Asymptotic functions and constants}

We define the scaled diffusion pattern vectors by

$\widehat{\boldsymbol{\imath}}_{+}=\lambda_{N}\left(\mathbf{I}-\widehat{\mathbf{R}}_{\infty}^{2}\right)^{-1} \widehat{\mathbf{k}}_{N}$,

$\widehat{\boldsymbol{\imath}}_{-}=\widehat{\mathbf{R}}_{\infty} \widehat{\boldsymbol{\iota}}_{+}$,

and note that for

$\widehat{\mathbf{Q}}_{+}=\left[\widehat{\mathbf{q}}_{1}^{+}, \ldots, \widehat{\mathbf{q}}_{N}^{+}\right]$,

we have the representations

$\widehat{\boldsymbol{\imath}}_{+}=\frac{1}{2}\left(\widehat{\mathbf{q}}_{N}^{+}+\lambda_{N} \mathbf{q}_{N}^{+}\right)$,

$\widehat{\boldsymbol{\iota}}_{-}=\widehat{\boldsymbol{\iota}}_{+}-\lambda_{N} \mathbf{q}_{N}^{+}$,

and the orthogonality relations

$\widehat{\mathbf{k}}_{N}^{T} \widehat{\boldsymbol{\iota}}_{+}=1$,

$\widehat{\mathbf{k}}_{N}^{T} \widehat{\boldsymbol{\iota}}_{-}=1$.

To derive the expressions of the functions that occur in the asymptotic theory, we introduce the discrete approximation to the escape function $\mathbf{k}_{N}$ by

$\mathbf{k}_{N}=\sqrt{\frac{\mathrm{k}}{2 \mathrm{~m}}} \mathbf{M}^{\frac{1}{2}} \mathbf{W}^{-\frac{1}{2}} \widehat{\mathbf{k}}_{N}$,

and the diffusion pattern vectors $\boldsymbol{\iota}_{ \pm}$by

$\boldsymbol{\iota}_{ \pm}=\sqrt{\frac{\mathrm{m}}{2 \mathrm{k}}} \mathbf{M}^{\frac{1}{2}} \mathbf{W}^{-\frac{1}{2}} \widehat{\boldsymbol{\iota}}_{ \pm}$.

As a result, the orthogonality relations for $\widehat{\boldsymbol{t}}_{+}$and $\widehat{\boldsymbol{t}}_{-}$yield

$2 \mathbf{k}_{N}^{T} \mathbf{W M}^{-1} \boldsymbol{\iota}_{+}=1$,

$2 \mathbf{k}_{N}^{T} \mathbf{W M}^{-1} \boldsymbol{\iota}_{-}=1$.

Essentially, the vectors $\boldsymbol{\iota}_{+}$and $\boldsymbol{\iota}_{-}$are the discrete approximations of the diffusion patterns $i(\mu)$ and $i(-\mu), \mu>0$, respectively. The constant $m$ in (409) and (410) is obtained by normalizing the diffusion pattern (cf. (197))

$\frac{1}{2} \int_{-1}^{1} i(\mu) \mathrm{d} \mu=\frac{1}{2} \mathbf{1}^{T} \mathbf{W}\left(\boldsymbol{\iota}_{+}+\boldsymbol{\iota}_{-}\right)=\frac{1}{2} \sqrt{\frac{\mathrm{m}}{2 \mathrm{k}}} \mathbf{1}^{T} \mathbf{W}^{\frac{1}{2}} \mathbf{M}^{\frac{1}{2}} \widehat{\mathbf{q}}_{N}^{+}=1$,

that is,

$\mathrm{m}=\frac{8 \mathrm{k}}{\left(\mathbf{1}^{T} \mathbf{W}^{\frac{1}{2}} \mathbf{M}^{\frac{1}{2}} \widehat{\mathbf{q}}_{N}^{+}\right)^{2}}$, 
where $\mathbf{1}$ is the vector of all ones. Obviously, (411) and (412) are the discrete approximations of the normalization conditions (200) and (202), respectively.

In terms of the escape function $\mathbf{k}_{N}$, the reflection and transmission matrices are given by

$$
\begin{aligned}
\mathbb{R} & =\mathbb{R}_{\infty}-\delta_{m 0} \mathbf{r} \mathbf{k}_{N} \mathbf{k}_{N}^{T}, \\
\mathbb{T} & =\delta_{m 0} \mathbf{t} \mathbf{k}_{N} \mathbf{k}_{N}^{T}, \\
\mathbb{R}_{\infty} & =\frac{1}{2} \mathbf{M}^{\frac{1}{2}} \mathbf{W}^{-\frac{1}{2}} \widehat{\mathbf{R}}_{\infty} \mathbf{W}^{-\frac{1}{2}} \mathbf{M}^{\frac{1}{2}},
\end{aligned}
$$

where

$\mathrm{r}=\widehat{\mathrm{r}} \frac{\mathrm{m}}{\mathrm{k}}=\frac{\mathrm{ml} \mathrm{e}^{-2 \mathrm{k} \bar{\tau}}}{1-\mathrm{l}^{2} \mathrm{e}^{-2 \mathrm{k} \bar{\tau}}}$,
$\mathrm{t}=\widehat{\mathrm{t}} \frac{\mathrm{m}}{\mathrm{k}}=\frac{\mathrm{me}^{-\mathrm{k} \bar{\tau}}}{1-\mathrm{l}^{2} \mathrm{e}^{-2 \mathrm{k} \bar{\tau}}}$.

Moreover, for the azimuthal mode $m=0$, we have

$$
\begin{aligned}
\mathbf{r}_{\infty} & =2 \mathbb{R}_{\infty} \mathbf{v}, \\
r_{s \infty} & =4 \mathbf{v}^{T} \mathbb{R}_{\infty} \mathbf{v},
\end{aligned}
$$

and

$$
\begin{aligned}
\mathbf{r} & =\mathbf{r}_{\infty}-\mathrm{nr} \mathbf{k}_{N}, \\
\mathbf{t} & =\mathrm{nt} \mathbf{k}_{N}, \\
r_{s} & =4 \mathbf{v}^{T} \mathbb{R} \mathbf{v},
\end{aligned}
$$

where $\mathbf{r}_{\infty}$ and $r_{s \infty}$ are the plane albedo vector and the spherical albedo of the semi-infinite atmosphere, respectively, $\mathbf{r}$, $\mathbf{t}$ and $r_{s}$ are the plane albedo vector, the transmission vector, and the spherical albedo of the layer, respectively, and $\mathbf{v}=\mathbf{W M}^{-1} \mathbf{1}$. The $\mu$-weighted mean of the escape function is given by

$\mathrm{n}=2 \mathbf{k}_{N}^{T} \mathbf{v}$

which is the discrete approximation of (206).

The reflection and transmission matrices of the homogeneous layer with an underlying Lambertian surface $\mathbb{R}_{A}$ and $\mathbb{T}_{A}$, respectively, as well as the plane albedo $\mathbf{r}_{A}$ and the spherical albedo $r_{S A}$ are computed as

$$
\begin{aligned}
\mathbb{R}_{A} & =\mathbb{R}+\frac{A}{1-A r_{s}} \delta_{m 0} \mathbf{t t}^{T}, \\
\mathbb{T}_{A} & =\mathbb{T}+\frac{A}{1-A r_{s}} \delta_{m 0} \mathbf{r t}^{T}, \\
\mathbf{r}_{A} & =2 \mathbb{R}_{A} \mathbf{W} \mathbf{M}^{-1} \mathbf{1} \\
r_{s A} & =4 \mathbf{v}^{T} \mathbb{R}_{A} \mathbf{v} .
\end{aligned}
$$

where $A$ is the ground albedo.

\section{References}

[1] S. Twomey, H. Jacobowitz, H. B. Howell, Matrix methods for multiplescattering problems, J. Atmos. Sci. 23 (3) (1966) 289-298. doi:10. $1175 / 1520-0469(1966) 023<0289: \mathrm{mmfmsp}>2$. 0 . co; 2 .
[2] V. V. Sobolev, D. ter Haar, Light Scattering in Planetary Atmospheres, Pergamon Press, 1975.

[3] J. E. Hansen, L. D. Travis, Light scattering in planetary atmospheres, Space Science Reviews 16 (4) (1974) 527-610. doi:10.1007/ bf 00168069 .

[4] W. M. Irvine, Multiple scattering in planetary atmospheres, Icarus 25 (2) (1975) 175-204. doi : 10.1016/0019-1035(75)90019-6.

[5] J. Lenoble, Radiative Transfer in Scattering and Absorbing Atmospheres: Standard Computational Procedures, Studies in geophysical optics and remote sensing, A. Deepak, 1985.

[6] K. Stamnes, The theory of multiple scattering of radiation in plane parallel atmospheres, Rev. Geophys. 24 (2) (1986) 299. doi:10.1029/ rg024i002p00299.

[7] V. Budak, D. Klyuykov, S. Korkin, Convergence acceleration of radiative transfer equation solution at strongly anisotropic scattering, in: A. A. Kokhanovsky (Ed.), Light scattering reviews, Vol. 5, Springer Berlin Heidelberg, 2010, pp. 147-203. doi : 10 .1007/978-3-642-10336-0_5.

[8] V. P. Afanas'ev, D. S. Efremenko, A. V. Lubenchenko, On the application of the invariant embedding method and the radiative transfer equation codes for surface state analysis, in: A. Kokhanovsky (Ed.), Light Scattering Reviews 8, Springer Science + Business Media, 2013, pp. 363-423. doi:10.1007/978-3-642-32106-1_8.

[9] V. Rozanov, A. Rozanov, A. Kokhanovsky, J. Burrows, Radiative transfer through terrestrial atmosphere and ocean: Software package SCIATRAN, J Quant Spectrosc Radiat Transf 133 (2014) 13-71. doi:10. 1016/j.jqsrt. 2013.07.004.

[10] V. Afanas'ev, D. Efremenko, P. Kaplya, Analytical and numerical methods for computing electron partial intensities in the case of multilayer systems, Journal of Electron Spectroscopy and Related Phenomena 210 (2016) 16-29. doi:10.1016/j.elspec.2016.04.006.

[11] N. Rogovtsov, F. Borovik, Application of general invariance relations reduction method to solution of radiation transfer problems, J Quant Spectrosc Radiat Transf 183 (2016) 128-153. doi:10.1016/j.jqsrt. 2016.06 .024$.

[12] G. C. Wick, Über ebene Diffusionsprobleme, Zeitschrift für Physik 121 (11-12) (1943) 702-718. doi:10.1007/BF01339167.

[13] S. Chandrasekhar, Radiative Transfer, Dover Publications, Inc., New York, 1950.

[14] K. Stamnes, R. A. Swanson, A New Look at the Discrete Ordinate Method for Radiative Transfer Calculations in Anisotropically Scattering Atmospheres, J Atmos Sci 38 (2) (1981) 387-389. doi:10.1175/ $1520-0469$ (1981) 038<0387: ANLATD>2 . 0. C0; 2 .

[15] G. N. Plass, G. W. Kattawar, F. E. Catchings, Matrix operator theory of radiative transfer 1: Rayleigh scattering, Appl. Opt. 12 (2) (1973) 314. doi:10.1364/ao.12.000314.

[16] G. W. Kattawar, G. N. Plass, F. E. Catchings, Matrix operator theory of radiative transfer 2: Scattering from maritime haze, Appl. Opt. 12 (5) (1973) 1071. doi:10.1364/ao.12.001071.

[17] H. van de Hulst, A new look at multiple scattering, Tech. report, NASA Inst. Space Studies (1963)

[18] T. Nakajima, M. Tanaka, Matrix formulations for the transfer of solar radiation in a plane-parallel scattering atmosphere, J Quant Spectrosc Radiat Transf 35 (1) (1986) 13-21. doi : 10 . 1016/0022-4073(86) 90088-9.

[19] V. Ambarzumian, Diffuse reflection of light by a foggy medium, Dokl. Akad. Nauk SSSR 38 (1943) 229-232.

[20] R. Bellman, R. Kalaba, G. Wing, Invariant imbedding and mathematical physics-i: Particle processes, J. Math. Phys. 1 (1960) 280-308. doi:10. 1063/1.1703663.

[21] P. C. Waterman, Matrix-exponential description of radiative transfer, Journal of the Optical Society of America 71 (4) (1981) 410. doi:10. 1364/josa.71.000410.

[22] P. J. Flatau, G. L. Stephens, On the fundamental solution of the radiative transfer equation, J. Geophys. Res. 93 (D9) (1988) 11037. doi: 10.1029/jd093id09p11037.

[23] V. P. Budak, D. A. Klyuykov, S. V. Korkin, Complete matrix solution of radiative transfer equation for PILE of horizontally homogeneous slabs, J Quant Spectrosc Radiat Transf 112 (7) (2011) 1141-1148. doi:10 . 1016/j.jqsrt.2010.08.028.

[24] V. P. Budak, D. S. Efremenko, O. V. Shagalov, Efficiency of algorithm for solution of vector radiative transfer equation in turbid medium slab, J. 
Phys.: Conf. Ser. 369 (2012) 012021. doi:10.1088/1742-6596/369/ $1 / 012021$.

[25] A. Doicu, T. Trautmann, Discrete-ordinate method with matrix exponential for a pseudo-spherical atmosphere: Scalar case, J Quant Spectrosc Radiat Transf 110 (1-2) (2009) 146-158. doi:10.1016/j . jqsrt. 2008.09 .014$.

[26] A. Doicu, T. Trautmann, Discrete-ordinate method with matrix exponential for a pseudo-spherical atmosphere: Vector case, J Quant Spectrosc Radiat Transf 110 (1-2) (2009) 159-172. doi:10.1016/j . jqsrt. 2008.09 .013$.

[27] T. Nakajima, M. D. King, Asymptotic theory for optically thick layers: application to the discrete ordinates method, Appl. Opt. 31 (36) (1992) 7669. doi:10.1364/ao.31.007669.

[28] D. Efremenko, A. Doicu, D. Loyola, T. Trautmann, Small-angle modification of the radiative transfer equation for a pseudo-spherical atmosphere, J Quant Spectrosc Radiat Transf 114 (2013) 82-90. doi: 10.1016/j.jqsrt.2012.08.013.

[29] D. Efremenko, A. Doicu, D. Loyola, T. Trautmann, Acceleration techniques for the discrete ordinate method, J Quant Spectrosc Radiat Transf 114 (2013) 73-81. doi:10.1016/j.jqsrt.2012.08.014.

[30] K. Stamnes, S. C. Tsay, T. Nakajima, Computation of eigenvalues and eigenvectors for the discrete ordinate and matrix operator methods in radiative transfer, J Quant Spectrosc Radiat Transf 39 (5) (1988) 415-419. doi:10.1016/0022-4073(88)90107-0.

[31] A. H. Karp, J. Greenstadt, J. A. Fillmore, Radiative transfer through an arbitrarily thick, scattering atmosphere, J Quant Spectrosc Radiat Transf 24 (5) (1980) 391-406. doi : 10.1016/0022-4073 (80)90074-6.

[32] E. G. Yanovitskij, Light Scattering in Inhomogeneous Atmospheres, Springer Nature, 1997. doi : 10.1007/978-3-642-60465-2.

[33] W. J. Wiscombe, The delta-M method: Rapid yet accurate radiative flux calculations for strongly asymmetric phase functions, J Atmos Sci 34 (9) (1977) 1408-1422. doi : 10.1175/1520-0469 (1977) 034<1408: TDMRYA $>2.0 . \mathrm{CO} ; 2$

[34] T. Nakajima, M. Tanaka, Algorithms for radiative intensity calculations in moderately thick atmos using a truncation approximation, J Quant Spectrosc Radiat Transf 40 (1) (1988) 51-69. doi:10.1016/ 0022-4073 (88) 90031-3.

[35] M. Mishchenko, J. Dlugach, E. Yanovitskij, N. Zakharova, Bidirectional reflectance of flat, optically thick particulate layers: an efficient radiative transfer solution and applications to snow and soil surfaces, J Quant Spectrosc Radiat Transf 63 (2-6) (1999) 409-432. doi:10.1016/ s0022-4073(99)00028-x.

[36] Y. Yang, A. Marshak, J. Mao, A. Lyapustin, J. Herman, A method of retrieving cloud top height and cloud geometrical thickness with oxygen $a$ and $b$ bands for the deep space climate observatory (DSCOVR) mission: Radiative transfer simulations, J Quant Spectrosc Radiat Transf 122 (2013) 141-149. doi:10.1016/j.jqsrt.2012.09.017.

[37] G. McGarragh, P. Gabriel, Efficient computation of radiances for optically thin media by Padé approximants, J Quant Spectrosc Radiat Transf 111 (12-13) (2010) 1885-1899. doi:10.1016/j.jqsrt.2010.03.011.

[38] C. V. Loan, Computing integrals involving the matrix exponential, IEEE Transactions on Automatic Control 23 (3) (1978) 395-404. doi:10. 1109/tac.1978.1101743.

[39] I. P. Grant, G. E. Hunt, Discrete space theory of radiative transfer. I. Fundamentals, Proceedings of the Royal Society A: Mathematical, Physical and Engineering Sciences 313 (1513) (1969) 183-197. doi:10.1098/ rspa.1969.0187.

40] W. Wiscombe, On initialization, error and flux conservation in the doubling method, J Quant Spectrosc Radiat Transf 16 (8) (1976) 637-658. doi: 10.1016/0022-4073(76)90056-x.

[41] A. K. Heidinger, C. O'Dell, R. Bennartz, T. Greenwald, The successiveorder-of-interaction radiative transfer model. Part I: Model development, J. Appl. Meteor. Climatol. 45 (10) (2006) 1388-1402. doi:10. $1175 /$ jam2387. 1.

[42] A. V. Rozanov, V. V. Rozanov, J. P. Burrows, Combined differentialintegral approach for the radiation field computation in a spherical shell atmosphere: Nonlimb geometry, Journal of Geophysical Research: Atmospheres 105 (D18) (2000) 22937-22942. doi:10.1029/ $2000 j d 900378$

[43] X. Sun, Y. Han, X. Shi, Application of asymptotic theory for computing the reflection of optically thick clouds, Journal of Optics A: Pure and Applied Optics 8 (12) (2006) 1074-1079. doi : 10.1088/1464-4258/8/ 12/007.

[44] M. King, S.-C. Tsay, S. Platnick, M. Wang, L. K.-N., Cloud retrieval algorithms for MODIS: Optical thickness, effective particle radius, and thermodynamic phase, Tech. rep. (1997).

URL http: //modis.gsf c.nasa.gov/data/atbd/atbd_mod05.pdf

[45] A. A. Kokhanovsky, V. V. Rozanov, The reflection function of optically thick weakly absorbing turbid layers: a simple approximation, J Quant Spectrosc Radiat Transf 77 (2) (2003) 165-175. doi:10.1016/ S0022-4073(02)00085-7.

[46] A. A. Kokhanovsky, Reflection of light from nonasbsorbing semi-infinite cloudy media: a simple approximation, J Quant Spectrosc Radiat Transf 85 (1) (2004) 25-33. doi : 10.1016/S0022-4073(03)00192-4.

[47] M. D. King, Determination of the scaled optical thickness of clouds from reflected solar radiation measurements, J. Atmos. Sci. 44 (13) (1987) 1734-1751. doi:10.1175/1520-0469(1987) 044<1734: dotsot>2.0.co;2.

[48] K. Kawabata, S. Ueno, The first three orders of scattering in vertically inhomogeneous scattering-absorbing media, Astrophys Space Sci 150 (2) (1988) 327-344. doi : 10.1007/bf00641728.

[49] H. wen Chang, T.-L. Wu, Numerical solutions of matrix riccati equations for radiative transfer in a plane-parallel geometry, Waves in Random Media 7 (1) (1997) 147-168. doi:10.1088/0959-7174/7/1/010.

[50] T. Kurosu, V. Rozanov, J. Burrows, Parameterization schemes for terrestrial water clouds in the radiative transfer model GOMETRAN, Journal of Geophysical Research: Atmospheres 102 (D18) (1997) 21809-21823. doi: 10.1029/97jd01044.

[51] L. Delves, J. Mohamed, Computational methods for integral equations, Cambridge University Press. Cambridge, 1985.

[52] G. E. Thomas, K. Stamnes, Radiative transfer in the Atmosphere and Ocean, Radiative transfer in the Atmosphere and Ocean. Cambridge University press , 1st edition, 1999.

[53] C. Clenshaw, A. Curtis, A method for numerical integration on an automatic computer, Numerische Mathematik 2 (1) (1960) 197-205. doi: $10.1007 / \mathrm{bf} 01386223$

[54] J. Weideman, L. Trefethen, The kink phenomenon in fejér and clenshaw-curtis quadrature, Numerische Mathematik 107 (4) (2007) 707727. doi:10.1007/s00211-007-0101-2. 